\title{
Some Aspects of Tachyon Field Cosmology
}

\author{
Kourosh Nozar: * $^{\circ}$ and Narges Rashidi \\ Department of Physics, Faculty of Basic Sciences, \\ University of Mazandaran, \\ P. O. Box 47416-95447, Babolsar, IRAN
}

( Dated: December 26, 2017)

\begin{abstract}
We study inflation, perturbations, non-gaussinity and late-time cosmological dynamics of a tachyon field both minimally and non-minimally coupled to gravity. By analyzing the parameters space of the model, the viability of the model in confrontation with recent observational data is considered. In a dynamical system technique, we study the phase space dynamics of both minimally and non-minimally coupled tachyon field. We find the fixed points (lines in our setup) and explore their stability. Also, we perform a statefinder diagnostic to both cases and show that the trajectories of the state finder pairs reach a stable state which is corresponding to a $\Lambda$ CDM scenario.
\end{abstract}

PACS numbers: $98.80 . \mathrm{Cq}, 95.36 .+\mathrm{x}$

Key Words: Inflation, Cosmological Perturbations, Non-Gaussinity, Accelerated Expansion, Tachyon Field, Dynamical System, Statefinder Diagnostic

\section{INTRODUCTION}

Despite the great successes of the standard big bang cosmology in confrontation with observational data, it suffers from some shortcomings such as the flatness, horizon and relics problems. It has been shown that an inflationary stage in the early time evolution of the universe can address successfully at least some of these problems. In a simple inflationary model, the universe is dominated with a scalar field called inflaton whose potential energy dominates over the kinetic term (the slow-roll conditions), followed by a reheating period [1, 2, 3, 4, 5, 6, 7, 8]. Inflation also provides a mechanism for production of density perturbations required to seed the formation of structures in the universe. But there are several problems with no concrete solutions in inflation paradigm such as natural realization of inflation in a fundamental theory, cosmological constant and dark energy problem, unexpected low power spectrum at large scales and egregious running of the spectral index [9]. Another unsolved problem in the spirit of the inflationary scenario is that yet we don't know how to integrate it with ideas of the particle physics. For example, we would like to identify the inflaton, the scalar field that drives inflation, with one of the known fields of particle physics. Also, it is important that the inflaton potential to be emerged naturally from underlying fundamental theory 6 . In this respect, tachyon fields associated with unstable D-branes could be responsible for inflation in early time.

Focusing on late-time dynamics, recent cosmological observations show that our universe is undergoing an accelerating phase of expansion and transition to the accelerated phase has been occurred in the recent cosmological past [10, 11, 12]. The simplest way to describe the accelerating phase of universe expansion is to adopt a

\footnotetext{
* knozari@umz.ac.ir

$\dagger$ n.rashidi@umz.ac.ir
}

cosmological constant in Einstein's field equations. However, there are some problems with cosmological constant that make it unfavorable, such as huge amount of finetuning required for its magnitude and other theoretical problems such as unknown origin and lake of dynamics [13, 14]. To explain this remarkable behavior of the universe, several theoretical approaches have been proposed, some of which can be seen in Refs. 15, 16, 17, 18, 19, 20, 21, 22, 23, 24, 25, 26, 27, 28, 29, 30, 31]. Specially, dark energy models based on scalar fields have attracted much attention in recent years, see for instance [15, 32, 33, 34]. The scalar field we are going to explore its cosmological dynamics is the tachyon field described by the DiracBorn-Infeld (DBI) action. Such a scalar field is associated with D-branes in string theory [35, 36, 37]. This field can be responsible for early time inflation in the history of the universe (see for instance [38, 39]) and also can be considered as a dark energy in the late time (see for instance [40, 41, 42]). As has been indicated in Ref. Gibbons], in the case that the tachyon condensate starts to roll down slowly the potential, a universe dominated by this field evolves smoothly from a phase of accelerated expansion to an era dominated by a nonrelativistic fluid. These features show that tachyon fields may provide suitable candidates to realize initial inflation and late time cosmic speed-up. This is the reason why we consider a tachyon field for our purposes.

Since scalar fields can interact with other fields, such as the gravitational sector of the theory, in the spirit of scalar-tensor theories we can consider a non-minimal coupling (NMC) of the tachyon field with intrinsic (Ricci) curvature. There are compelling reasons to include an explicit non-minimal coupling in the action. For instance, it is necessary for the renormalizability of the scalar field theory in curved space. Also it arises at the quantum level when quantum corrections to the scalar field theory are considered. In most theories used to describe inflationary scenarios, a non-vanishing value of the coupling constant cannot be avoided [43, 44]. Many authors have 
studied the models with scalar field non-minimally coupled to gravity (see for instance [45, 46, 47, 48, 49, 50, 51, 52, 53, 54, 55]). The non-minimally coupled tachyon field also has been considered by some authors [56, 57, 58]. In this paper, we study cosmological dynamics of a tachyon field both in the inflationary and late time epochs. Firstly, we consider a tachyon field which is minimally coupled to the Ricci scalar. We study its cosmological dynamics as a field responsible for cosmic inflation. The perturbation will be considered in details and a comparison with recent observational data will be done. Also the issue of non-gaussinity of perturbations will be considered with details. Then we study the role of a tachyon field as a dark energy in the late time history of the universe. We study the cosmological dynamics of the model in a dynamical system approach. By obtaining the autonomous system of equations we consider if there is any late time attractor in the model. Also, by studying the cosmological diagnostic pair (dubbed statefinder) we compare the model, which contains a tachyon as dark energy component, with $\Lambda \mathrm{CDM}$ scenario. Secondly, we consider a tachyon field which is non-minimally coupled to the Ricci scalar. As the minimal case, we study the role of tachyon field as deriver of inflation and also a possible candidate for dark energy responsible for late time acceleration. Here also, the perturbations and their non-gaussinity will be studied in details. On the other hand, in a dynamical system technique and phase space trajectories we study the cosmological dynamics in the presence of a non-minimally coupled tachyon field. As we will show, in the non-minimal coupling case we find an attractor in the phase space of the model which has capability to explain the late time accelerating phase of the universe expansion. By exploring the diagnostic pair we shall see that a model with a non-minimally coupled tachyon field reaches a stable $\Lambda$ CDM-like state and remains there forever.

\section{MINIMALLY COUPLED TACHYON FIELD}

\section{A. Inflation}

The 4-dimensional action for a tachyon field, which is minimally coupled to gravity, can be written as follows

$$
S=\int \sqrt{-g}\left[\frac{1}{\kappa^{2}} R-V(\phi) \sqrt{1-\partial^{\mu} \phi \partial_{\mu} \phi}\right] d^{4} x
$$

where $R$ is the 4-dimensional Ricci scalar, $\phi$ is the tachyon field and $V(\phi)$ is its potential. Einstein's field equations calculated from action (1) are given by

$$
G_{\mu \nu}=\kappa^{2} T_{\mu \nu}
$$

where $T_{\mu \nu}$ is the energy momentum tensor given by

$T_{\mu \nu}=-\frac{V(\phi)}{\sqrt{1-\partial_{\alpha} \phi \partial^{\alpha} \phi}} \partial_{\mu} \phi \partial_{\nu} \phi+g_{\mu \nu}\left(-V(\phi) \sqrt{1-\partial_{\alpha} \phi \partial^{\alpha} \phi}\right)$.
The energy momentum tensor (3) leads to the following energy density and pressure for the tachyon field

$$
\rho_{\phi}=\frac{V(\phi)}{\sqrt{1-\dot{\phi}^{2}}}
$$

and

$$
p_{\phi}=-V(\phi) \sqrt{1-\dot{\phi}^{2}} .
$$

In order to formulate cosmological dynamics, we assume the following spatially flat FRW line element

$$
d s^{2}=-n^{2}(t) d t^{2}+a^{2}(t) \gamma_{i j} d x^{i} d x^{j}
$$

where $\gamma_{i j}$ is a maximally symmetric 3 -dimensional metric defined as $\gamma_{i j}=\delta_{i j}+k \frac{x_{i} x_{j}}{1-k r^{2}}$ where $k=-1,0,+1$ parameterizes the spatial curvature and $r^{2}=x_{i} x^{i}$. By using this line element with $n^{2}(t)=1$ and considering the $(0,0)$ component of Einstein's field equations, the Friedmann equation of a model with minimally coupled tachyon field and flat spatial section is obtained as follows

$$
H^{2}=\frac{\kappa^{2}}{3} \frac{V(\phi)}{\sqrt{1-\dot{\phi}^{2}}}
$$

Variation of the action (11) with respect to the scalar field gives the following equation of motion

$$
\frac{\ddot{\phi}}{1-\dot{\phi}^{2}}+3 H \dot{\phi}+\frac{V^{\prime}}{V}=0
$$

where a prime refers to a derivative with respect to the tachyon field and a dot marks derivative with respect to the cosmic time. Also, the energy conservation equation of the model is given by

$$
\dot{\rho}_{\phi}+3 H\left(\rho_{\phi}+p_{\phi}\right)=0 \text {. }
$$

During the inflationary era and in the slow-roll approximation, where $\dot{\phi}^{2} \ll 1$ and $\ddot{\phi} \ll|3 H \dot{\phi}|$, energy density and equation of motion for scalar field take the following forms respectively

$$
\rho_{\phi}=V(\phi)
$$

and

$$
3 H \dot{\phi}+\frac{V^{\prime}}{V}=0 .
$$

So, the Friedmann equation is reduced to the following form

$$
H^{2}=\frac{\kappa^{2}}{3} V(\phi) .
$$

Now, we introduce the slow-roll parameters as follows

$$
\epsilon \equiv-\frac{\dot{H}}{H^{2}},
$$


and

$$
\eta \equiv-\frac{1}{H} \frac{\ddot{H}}{\dot{H}}
$$

which in our setup and within the slow-roll approximation these parameters become

$$
\epsilon=\frac{1}{2 \kappa^{2}} \frac{V^{\prime 2}}{V^{3}},
$$

and

$$
\eta=\frac{1}{\kappa^{2}}\left(\frac{V^{\prime \prime}}{V^{2}}-\frac{1}{2} \frac{V^{\prime 2}}{V^{3}}\right) .
$$

The condition under which the inflation epoch could take place is $\{\epsilon, \eta\}<1$; as soon as one of these parameters reaches unity, the inflation phase terminates.

The number of e-folds during inflation is given by

$$
N=\int_{t_{h c}}^{t_{f}} H d t
$$

which, within the slow-roll approximation can be written as

$$
N \simeq-\int_{\phi_{h c}}^{\phi_{f}} 3 H^{2} \frac{V}{V^{\prime}} d \phi,
$$

where $\phi_{h c}$ denotes the value of $\phi$ when the universe scale observed today crosses the Hubble horizon during inflation and $\phi_{f}$ is the value of $\phi$ when the universe exits the inflationary phase. In a model with a minimally coupled tachyon field, the number of e-folds in the slow-roll approximation takes the following form

$$
N \simeq-\int_{\phi_{h c}}^{\phi_{f}} \kappa^{2} \frac{V^{2}}{V^{\prime}} d \phi .
$$

The key test of any inflation model is the spectrum of perturbations produced due to quantum fluctuations of the fields about their homogeneous background values. So, in the following section, we consider the scalar perturbation of the metric in our setup.

\section{B. Perturbations}

In this section, we explore the linear perturbation theory in inflation with tachyon field. There are many different ways of characterizing cosmological perturbations, which depend on the choice of gauge (coordinates). In longitudinal gauge, the scalar metric perturbations of the FRW background are given by [59, 60, 61]

$$
d s^{2}=-(1+2 \Phi) d t^{2}+a^{2}(t)(1-2 \Psi) \delta_{i j} d x^{i} d x^{j},
$$

where $a(t)$ is the scale factor, $\Phi=\Phi(t, x)$ and $\Psi=$ $\Psi(t, x)$, the metric perturbations, are gauge-invariant variables. The spatial dependence of all perturbed quantities are of the form of plane waves $e^{i k x}$, where $k$ is the wave number. The perturbation of the metric leads to the perturbation in the energy-momentum tensor through Einstein's field equations. For the perturbed metric (20), the perturbed Einstein's field equations can be obtained as follows

$$
-3 H(H \Phi+\dot{\Psi})-\frac{k^{2}}{a^{2}}=\frac{\kappa_{4}^{2}}{2} \delta \rho_{\phi},
$$

$$
\begin{gathered}
\ddot{\Psi}+3 H(H \Phi+\dot{\Psi})+H \dot{\Phi}+2 \dot{H} \Phi+\frac{1}{3 a^{2}} k^{2}(\Phi-\Psi)=\frac{\kappa_{4}^{2}}{2} \delta p_{\phi}, \\
\dot{\Psi}+H \Phi=-\frac{\kappa^{2} V(\phi)}{\sqrt{1-\dot{\phi}^{2}}} \frac{\dot{\phi} \delta \phi}{2} . \\
\Psi-\Phi=0 .
\end{gathered}
$$

As we see from last equation, in the minimally coupled tachyon field model, the two metric perturbations are equal. In equations (21) and (22), $\delta \rho_{\phi}$ and $\delta p_{\phi}$, the perturbed energy density and pressure are given by

$$
\delta \rho_{\phi}=\frac{V^{\prime} \delta \phi}{\sqrt{1-\dot{\phi}^{2}}}-V \frac{\dot{\phi} \delta \dot{\phi}+\dot{\phi}^{2} \Phi}{\left(1-\dot{\phi}^{2}\right)^{\frac{3}{2}}},
$$

and

$$
\delta p_{\phi}=-V^{\prime} \sqrt{1-\dot{\phi}^{2}} \delta \phi-V \frac{\dot{\phi} \delta \dot{\phi}+\dot{\phi}^{2} \Phi}{\sqrt{1-\dot{\phi}^{2}}},
$$

which are obtained by perturbing of equations (4) and (5). The variation of the scalar field's equation of motion (8) leads to the following expression

$$
\begin{aligned}
\delta \ddot{\phi}+3 H \delta \dot{\phi}+2 \dot{\phi} \ddot{\phi} \frac{\delta \dot{\phi}+\dot{\phi}^{2} \Phi}{1-\dot{\phi}^{2}}+\frac{\sqrt{1-\dot{\phi}^{2}}}{V}\left[\left(\frac{k a^{2}}{a^{2}}\right.\right. \\
-3 \dot{H}) \Phi-\frac{2 k^{2}}{a^{2}} \Psi-3(\ddot{\Psi}+4 H \dot{\Psi}+H \dot{\Phi} \\
\left.\left.+\dot{H} \Phi+4 H^{2} \Phi\right)\right]=\left(6 H \dot{\phi}^{3}-\frac{2 V^{\prime}}{V}\left(1-\dot{\phi}^{2}\right)\right) \Phi \\
+\dot{\phi}(\dot{\Phi}+3 \dot{\Psi})+\left(1-\dot{\phi}^{2}\right) \delta \phi\left(\frac{V^{\prime \prime}}{V}-\frac{V^{\prime 2}}{V^{2}}\right) .
\end{aligned}
$$

One can decompose the scalar perturbations into the entropy (isocurvature) perturbations which are projection orthogonal to the trajectory, and adiabatic (curvature) perturbations which are projection parallel to the trajectory. If inflation is driven by more than one scalar field 62, 63, 64, 65] or it interacts with other fields such as the scalar Ricci term 66, 67], we deal with the isocurvature perturbations. If there is only one scalar 
field during the inflationary period, we deal with the adiabatic perturbations [64, 65, 66, 67, 68]. In this section, since the tachyon field is the only field in the inflationary period, the perturbations are adiabatic perturbations. Since we are dealing with the linear perturbation (first order cosmological perturbations), we can define a gauge-invariant primordial curvature perturbation $\zeta$, on scales outside the horizon, as follows [69]

$$
\zeta=\Psi-\frac{H}{\dot{\rho}_{\phi}} \delta \rho_{\phi} .
$$

On uniform density hypersurfaces where $\delta \rho_{\phi}=0$, the above quantity reduces to the curvature perturbation, $\Psi$. Equation (28) leads to the following equation for time evolution of $\zeta$, 70]

$$
\dot{\zeta}=H\left(\frac{\delta p_{n a d}}{\rho_{\phi}+p_{\phi}}\right) .
$$

Equation (29) shows that the change in the curvature perturbation on uniform-density hypersurfaces, on large scales, is due to the non-adiabatic part of the pressure perturbation, independent of the form of the gravitational field equations. $\zeta$ is constant if the pressure perturbation is adiabatic on the large scales.

The pressure perturbation (in any gauge) can be decomposed into adiabatic and entropic (non-adiabatic) parts [0]

$$
\delta p=c_{s}^{2} \delta \rho_{\phi}+\dot{p}_{\phi} \Gamma
$$

where $c_{s}^{2}=\frac{\dot{p}_{\phi}}{\dot{\rho}_{\phi}}$ is the sound effective velocity. The nonadiabatic part is $\delta p_{n a d}=\dot{p}_{\phi} \Gamma$, where $\Gamma$ marks the displacement between hypersurfaces of uniform pressure and density. From equations (30) and within the slow-roll conditions, we can deduce

$$
\delta p_{\text {nad }}=0
$$

That is, the non-adiabatic part of the pressure perturbation is zero; the pressure perturbation is adiabatic. So, from equation (29) we find

$$
\dot{\zeta}=0
$$

It has been shown that the curvature perturbation on uniform density hypersurfaces, in terms of the scalar field fluctuations on spatially flat hypersurfaces, is given by [ $]$

$$
\zeta=-\frac{H \delta \phi}{\dot{\phi}} .
$$

Also, the field fluctuations at Hubble crossing and within the slow-roll limit are given by the following expression, which is independent of the underlying gravity theory for a massless field in de Sitter space

$$
\left\langle\delta \phi^{2}\right\rangle=\frac{H^{2}}{4 \pi^{2}} .
$$

Since in the minimally coupled tachyon field setup, perturbations are adiabatic, $\zeta$ can be related to the density perturbations by the following equation 71

$$
A_{s}^{2}=\frac{\left\langle\zeta^{2}\right\rangle}{V}
$$

So, from equations (33)-(35), we find

$$
A_{s}^{2}=\frac{\kappa^{6}}{12 \pi^{2}} \frac{V^{4}}{V^{\prime 2}} .
$$

The scale-dependence of the perturbations is described by the spectral index as

$$
n_{s}-1=\frac{d \ln A_{s}^{2}}{d \ln k}
$$

The interval in wave number is related to the number of e-folds by the relation

$$
d \ln k(\varphi)=d N(\varphi)
$$

So, from equations (36)-38) we obtain (by regarding the definition of the slow-roll parameters)

$$
n_{s}=1-6 \epsilon+2 \eta
$$

The tensor perturbations amplitude of a given mode when leaving the Hubble radius are given by

$$
A_{T}^{2}=\left.\frac{4 \kappa^{2}}{25 \pi} H^{2}\right|_{k=a H}
$$

In our setup and within the slow-roll approximation, we find

$$
A_{T}^{2}=\frac{4 \kappa^{4}}{75 \pi} V
$$

The tensor spectral index is given by

$$
n_{T}=\frac{d \ln A_{T}^{2}}{d \ln k},
$$

that in our model and in terms of the slow-roll parameters, the tensor (gravitational wave) spectral index is given by the following expression

$$
n_{T}=-2 \epsilon
$$

Another important parameter is the ratio between the amplitudes of tensor and scalar perturbations (tensor-toscalar ratio) which is given by

$$
r_{t-s}=\frac{A_{T}^{2}}{A_{s}^{2}}=\frac{32}{25 \pi} \epsilon .
$$

In the following we perform numerical analysis in our setup, by considering three types of potential. 

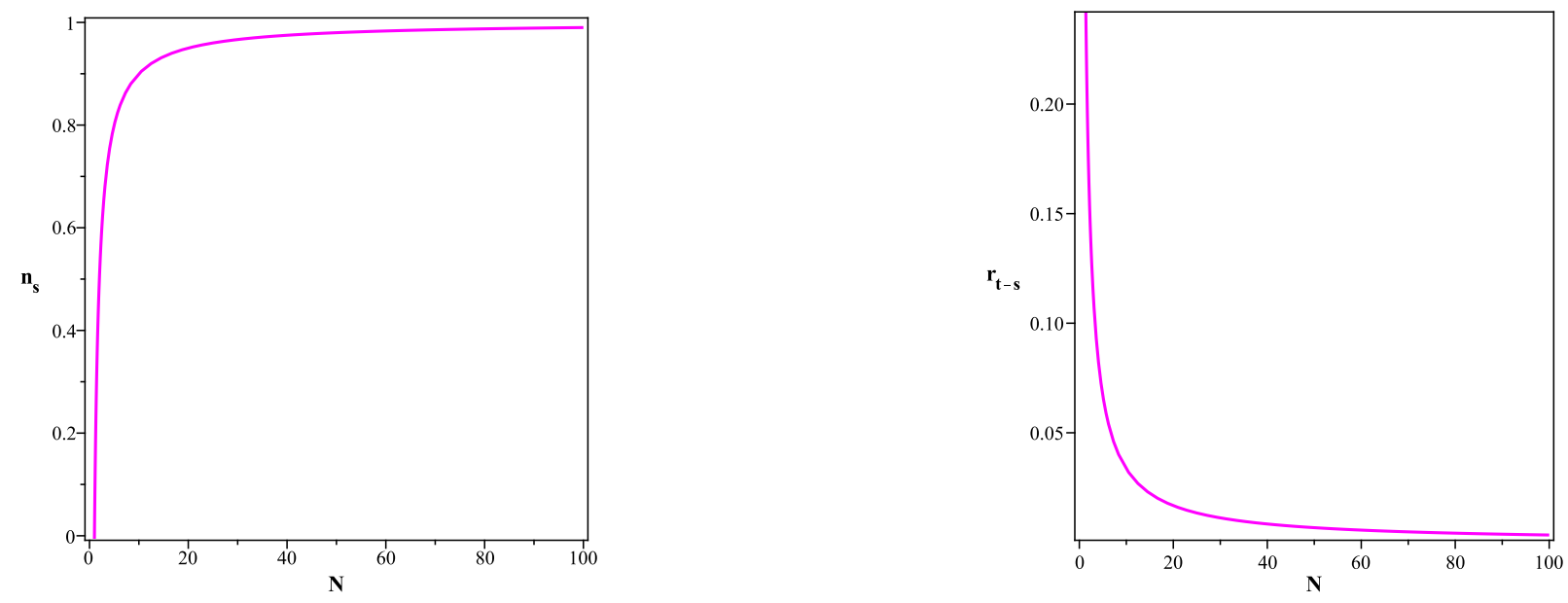

FIG. 1. Evolution of the scalar spectral index (left panel) and tensor to scalar ratio (right panel) versus the number of e-folds for the quadratic potential. For a minimally coupled tachyon field, the spectral index is red tilted and nearly scale invariant.

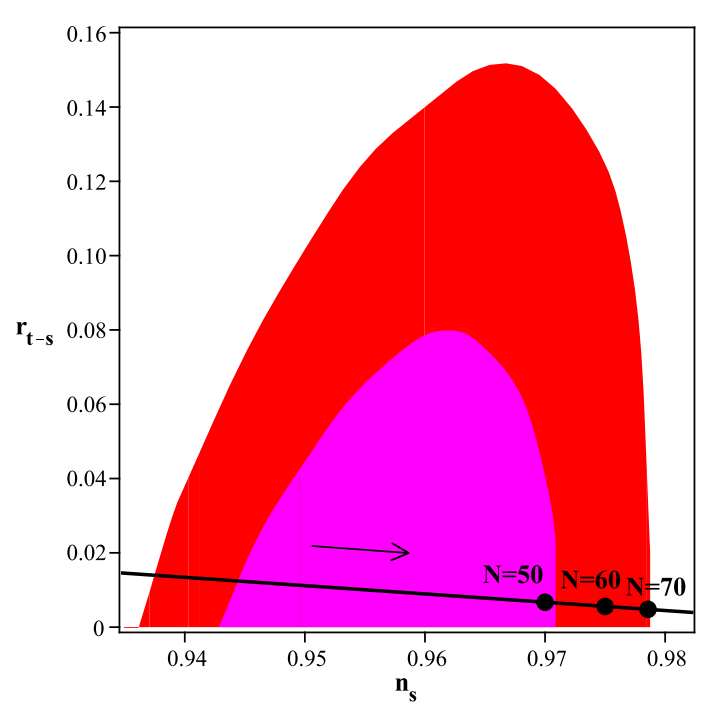

FIG. 2. Behavior of the tensor to scalar ratio with respect to the scalar spectral index for various $N$ and for quadratic potential in the background of WMAP9+eCMB $+\mathrm{BAO}+\mathrm{H}_{0}$ data. The two contours are corresponding to the $68 \%$ and $95 \%$ levels of confidence. $N$ increases in the direction of the arrow.

$$
\text { 1. } V=\frac{1}{2} \sigma \phi^{2}
$$

In this subsection, we consider the quadratic tachyon potential. With this choice, solving the integral of equation (19) gives

$$
N=-\frac{1}{16} \kappa^{2} \sigma\left(\phi_{f}^{4}-\phi_{h c}^{4}\right) .
$$

By assuming $\phi_{h c} \gg \phi_{f}$, we find $\phi_{h c}$ from equation (45). Then we substitute this obtained parameter into equation (39) and plot the evolution of the scalar spectral index versus the number of e-folds parameter. One can see the result in the left panel of figure 1, By increasing the number of e-folds parameter, the scalar spectral index increases and reaches unity asymptotically. As figure shows, in the case of minimally coupled tachyon field, the scalar spectral index is red-tilted and nearly scale invariant (note that in all figures we have set $\kappa=\sigma=1$ ). The evolution of the tensor to scalar ratio with respect to the number of e-folds parameter is shown in the right panel of the figure 1. The value of this ratio decreases as $N$ increases. To compare with observational data, we plot the evolution of the tensor to scalar ratio versus the scalar spectral index for various $N$ in the background of WMAP9+eCMB+BAO $+\mathrm{H}_{0}$ data (figure 2) (see ref [72]). Note that the observational parameters are defined at $k_{0}=0.002 \mathrm{Mpc}^{-1}$ where $k_{0}$ refers to the value of $k$ when universe scale crosses the Hubble horizon during inflation. In the case of quadratic potential, for $24 \leq N \leq 70$ the values of $r_{t-s}$ and $n_{s}$ are compatible with observational data. We see that for these values, the scalar spectral index, at the time that physical scales crossed the horizon, is red-tilted and nearly scale invariant. In the plot we have highlighted three values of $N$ that are considered usually in literature $(N=50, N=60, N=70)$.

$$
\text { 2. } V=V_{0} e^{-\kappa \sigma \phi}
$$

Another potential which is considered in this paper, is an exponential type potential. With an exponential potential, solving the integral of equation (19) gives

$$
N=\frac{V_{0}\left(e^{-\kappa \sigma \phi_{h c}}-e^{-\kappa \sigma \phi_{f}}\right)}{\sigma^{2}} .
$$

If we set (15) equal to 1 (corresponding to the end of inflation), we obtain $\phi_{f}$ and by substituting it in equation (46) we find $\phi_{h c}$. Now, we substitute this obtained parameter into equation (39) and plot the evolution of the scalar 

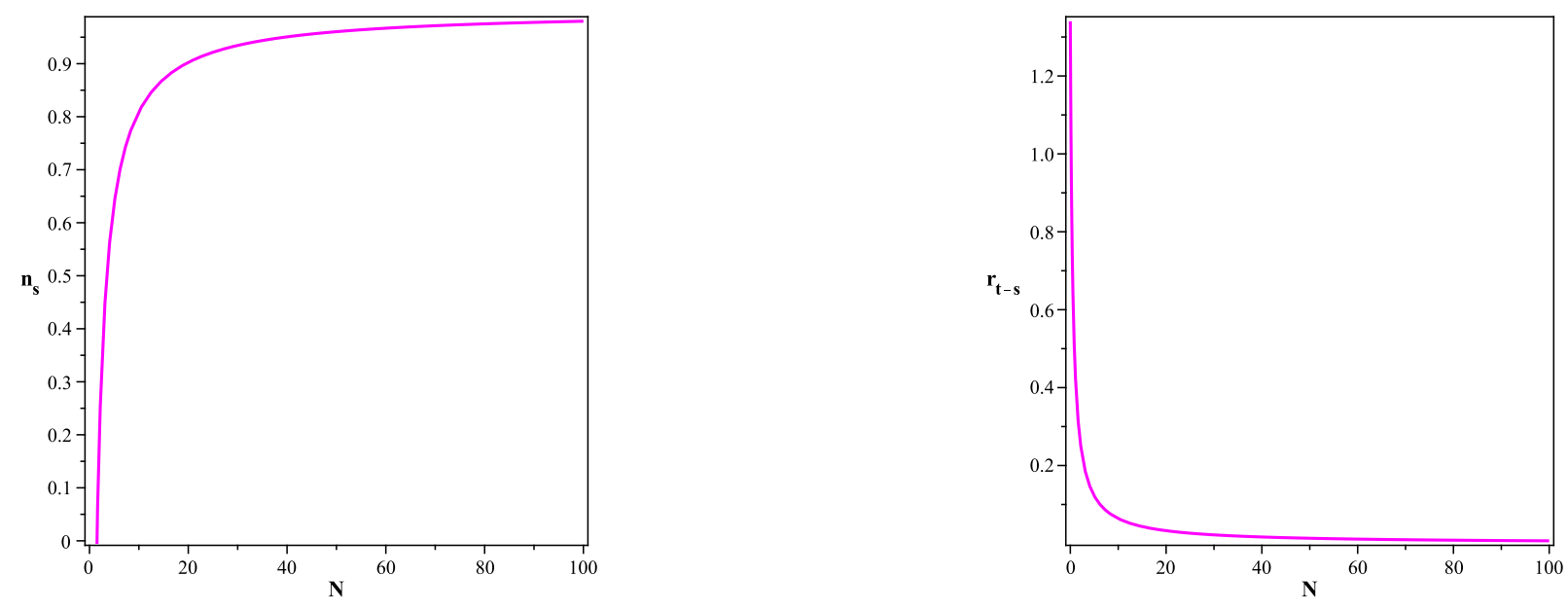

FIG. 3. Evolution of the scalar spectral index (left panel) and tensor to scalar ratio (right panel) versus the number of e-folds, for an exponential type potential.

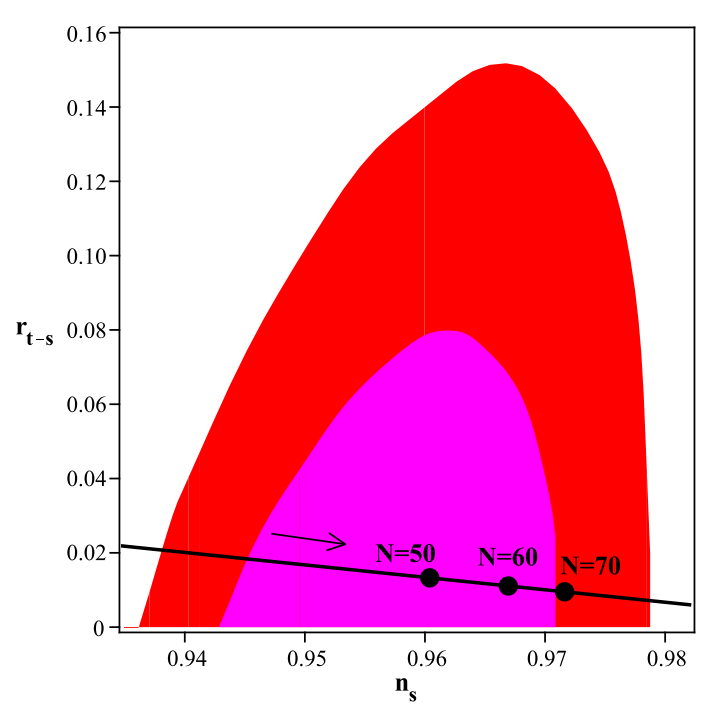

FIG. 4. Behavior of the tensor to scalar ratio with respect to the scalar spectral index for various $N$ and for exponential type potential in the background of $\mathrm{WMAP} 9+\mathrm{eCMB}+\mathrm{BAO}+\mathrm{H}_{0}$ data. The two contours are corresponding to the $68 \%$ and $95 \%$ levels of confidence.

spectral index versus the number of e-folds (left panel of figure 3). As figure shows, by increasing the number of efolds parameter, the scalar spectral index increases and also, it is red-tilted. The right panel of figure 3 shows the evolution of the tensor to scalar ratio with respect to the number of e-folds parameter. The value of this ratio decreases as $N$ increases. Here also, to compare with observational data, we plot the evolution of the tensor to scalar ratio versus the scalar spectral index for various $N$ in the background of WMAP9 $+\mathrm{eCMB}+\mathrm{BAO}+\mathrm{H}_{0}$ data in figure 4 . In the case of exponential potential, for $31 \leq N \leq 90$ the values of $r_{t-s}$ and $n_{s}$ are compatible with observational data. Here also, we have highlighted three values of $N ; N=50, N=60$ and $N=70$. In table \we have summarized the value of $n_{s}$ and $r_{t-s}$ for the mentioned values of $N$ and both for quadratic and exponential potentials.

\section{Intermediate inflation}

Intermediate inflation is an interesting scenario in which the scale factor evolves slower than the standard de Sitter inflation $(a=\exp (H t))$ and faster than the power law inflation $\left(a=t^{p}\right.$ with $\left.p>1\right)$. The evolution of the scale factor in an intermediate inflation is given by $a=a_{0} \exp \left(\vartheta t^{l}\right)$ with $0<l<1$ and positive constant $\vartheta$. With this scale factor and by using equations (12) and (13) we find the intermediate potential as follows

$$
V=b \phi^{-\beta}
$$

where

$$
\beta=\frac{4 l-4}{l-2},
$$

and

$$
b=\frac{3 \vartheta^{2} l^{2}}{\kappa^{2}}\left(\frac{8}{3} \frac{1-l}{(l-2)^{2} \vartheta l}\right)^{\frac{2 l-2}{l-2}} .
$$

With intermediate potential (47) we solve the integral of equation (19) to obtain

$$
N=-\frac{\left(\phi_{h c}^{-\beta+2}-\phi_{f}^{-\beta+2}\right) \kappa^{2} b}{(\beta-2) \beta} .
$$

By finding $\phi_{f}$ from equation (15) and substituting it in equation (50) we obtain $\phi_{h c}$. Then, we substitute this obtained parameter into equation (39) and plot the evolution of the scalar spectral index versus the number of 
TABLE I. Comparing the value of inflationary parameters in the horizon crossing, for quadratic and exponential potential. Note that $r<0.13(95 \% \mathrm{CL})$ and $n_{s}=0.9636 \pm 0.0084$ with WMAP9+eCMB+BAO $+\mathrm{H}_{0}$.

\begin{tabular}{ccccc}
\hline \hline Potential & Inflationary parameter & $N=50$ & $N=60$ & $N=70$ \\
\hline$V=\sigma \phi^{2}$ & $n_{s}$ & 0.97 & 0.975 & 0.97857 \\
& $r$ & 0.0067020 & 0.0055851 & 0.0047872 \\
\hline$V=V_{0} e^{-\kappa \sigma \phi}$ & $n_{s}$ & 0.96040 & 0.96694 & 0.97163 \\
& $r$ & 0.013271 & 0.011078 & 0.0095065 \\
\hline \hline
\end{tabular}
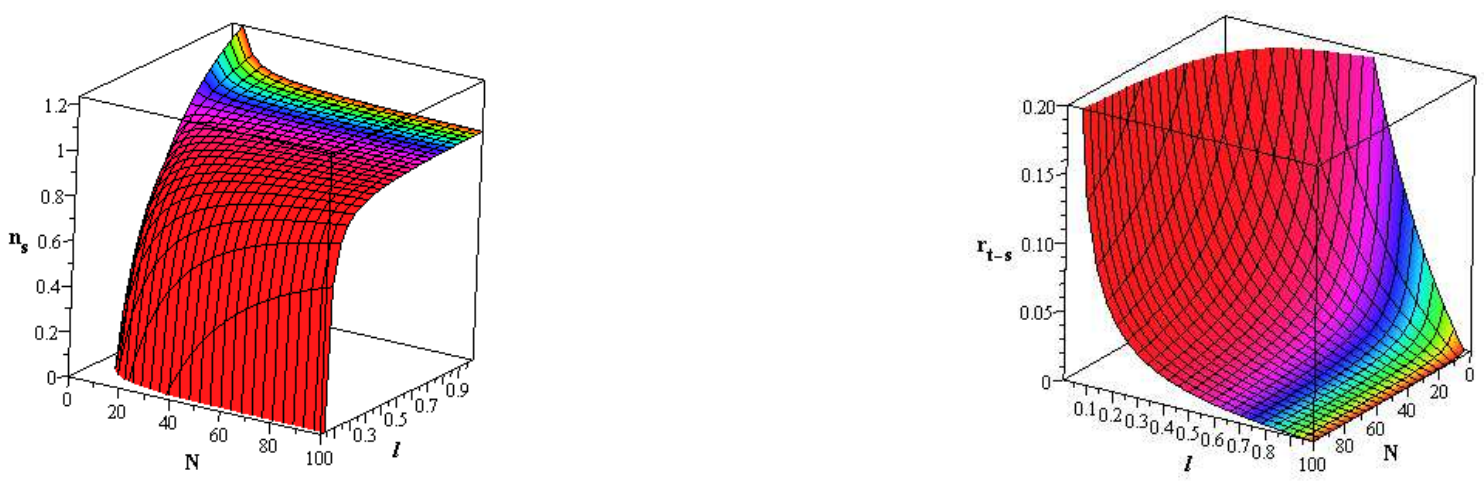

FIG. 5. Evolution of the scalar spectral index (left panel) and tensor to scalar ratio (right panel) versus the number of e-folds and the intermediate parameter $l$, for the intermediate potential.

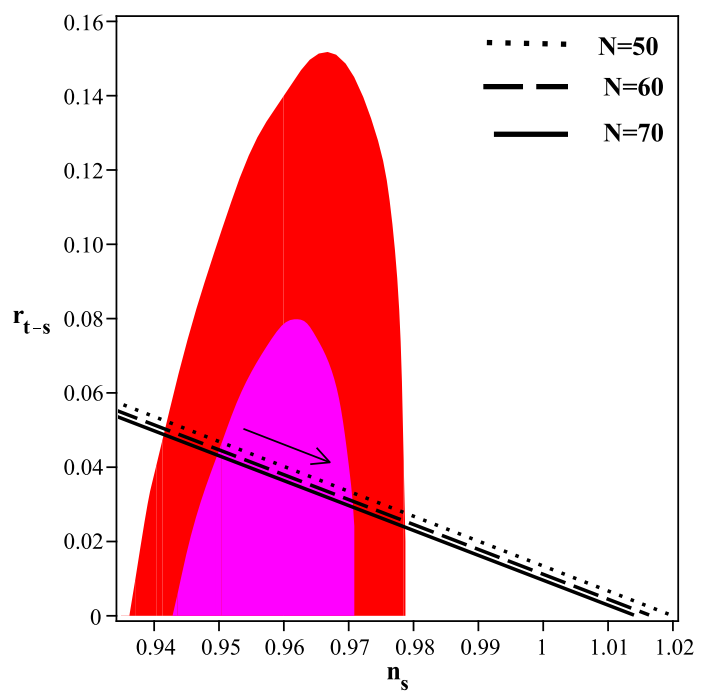

FIG. 6. Behavior of the tensor to scalar ratio with respect to the scalar spectral index for various $l$ for intermediate potential in the background of WMAP9 $+\mathrm{eCMB}+\mathrm{BAO}+\mathrm{H}_{0}$ data. The two contours are corresponding to the $68 \%$ and $95 \%$ levels of confidence. $l$ increases in the direction of the arrow.

$N$ and $l$ (left panel of figure 51). As figure shows, depending on the value of the number of e-folds parameter, the scalar spectral index is red-tilted for some values of $l$.
The right panel of figure 5 shows the evolution of the tensor to scalar ratio with respect to the number of e-folds and intermediate parameter, $l$. The value of this ratio decreases as $N$ and $l$ increase. Figure 6 shows the evolution of the tensor to scalar ratio versus the scalar spectral index in the background of WMAP9 $+\mathrm{eCMB}+\mathrm{BAO}+\mathrm{H}_{0}$ data for various $l$ and three values of $N(N=50, N=60$ and $N=70)$. Note that a similar figure has been plotted in ref [73] for three specified values of $l$ but various $N$. Here, we fix the value of $N$ and find the range of $l$ in which the model is compatible with observational data (see table II).

\section{Non-Guassianity}

Now we focus on the issue of non-gaussianity in the context of a minimally coupled tachyon field model by exploring the bispectrum or three-point correlation functions of the density perturbation distribution in Fourier space. In this regard we follow the reference [74]. Equation of motion of the tachyon field, by considering the gradient term, is given by the following expression

$$
\dot{\phi}=\frac{1}{3 H}\left[a^{-2} \nabla^{2} \phi-\frac{V^{\prime}}{V}+\Xi\right]
$$

where $\Xi$ is a stochastic term [8]. Now, to expand equation (51), the background tachyon field is treated with 
TABLE II. The range of intermediate parameter for which the values of inflationary parameters are compatible with observational data from WMAP9+eCMB $+\mathrm{BAO}+\mathrm{H}_{0}$.

\begin{tabular}{lccc}
\hline \hline & $N=50$ & $N=60$ & $N=70$ \\
\hline$l$ & $0.325<l<0.489$ & $0.295<l<0.463$ & $0.269<l<0.44$ \\
\hline \hline
\end{tabular}

fluctuations, $\phi(x, t)=\phi_{0}(t)+\delta \phi(x, t)$, with $\delta \phi=\delta \phi^{(1)}+$ $\delta \phi^{(2)}$. So, equation (51), in a time interval $t_{n}-t_{n-1}=\frac{1}{H}$, gives the following expressions for the first and second order fluctuations

$$
\frac{d}{d t}\left(\delta \phi^{(1)}(\mathbf{k}, t)\right)=\frac{1}{3 H}\left\{\left[-k^{2}-\frac{V^{\prime \prime}}{V}+\frac{V^{\prime 2}}{V^{2}}\right] \delta \phi^{(1)}+\Xi\right\}
$$

$$
\begin{aligned}
& \frac{d}{d t}\left(\delta \phi^{(2)}(\mathbf{k}, t)\right)=\frac{1}{3 H}\left\{\left[-k^{2}-\frac{V^{\prime \prime}}{V}+\frac{V^{\prime 2}}{V^{2}}\right] \delta \phi^{(2)}\right. \\
& \left.\quad+\left[-\frac{V^{\prime \prime \prime}}{V}+\frac{3 V^{\prime} V^{\prime \prime}}{V^{2}}-\frac{2 V^{\prime 3}}{V^{3}}\right]\left(\delta \phi^{(1)}\right)^{2}\right\}
\end{aligned}
$$

Note that, in order to focus on the computation of those non-Gaussian features which are directly produced by non-linearities (self-interactions) of the scalar field itself, we have perturbed the tachyon field's equation of motion in a particular gauge where metric perturbations can be neglected compared with those of the scalar field itself 75$]$.

The solution of the equations (52) and (53) are given by

$$
\begin{aligned}
& \delta \phi^{(1)}(\mathbf{k}, t)=\mathcal{F}\left(k, t-t_{n-1}\right) \int_{t_{n-1}}^{t} d t^{\prime} \frac{\Xi}{3 H} \mathcal{F}^{-1}\left(k, t-t_{n-1}\right) \\
& +\mathcal{F}\left(k, t-t_{n-1}\right) \delta \phi^{(1)}\left(\mathbf{k} e^{-H\left(t_{n}-t_{n-1}\right)}, t_{n-1}\right),
\end{aligned}
$$

and

$$
\begin{array}{r}
\delta \phi^{(2)}(\mathbf{k}, t)=\mathcal{F}\left(k, t-t_{n-1}\right) \int_{t_{n-1}}^{t} d t^{\prime} \mathcal{G}\left(k, t^{\prime}\right)\left[\int \frac{d^{3} p}{(2 \pi)^{3}} \delta \phi^{(1)}\left(\mathbf{p}, t^{\prime}\right) \delta \phi^{(1)}\left(\mathbf{k}-\mathbf{p}, t^{\prime}\right)\right] \mathcal{F}^{-1}\left(k, t^{\prime}-t_{n-1}\right) \\
+\mathcal{F}\left(k, t-t_{n-1}\right) \delta \phi^{(2)}\left(\mathbf{k} e^{-H\left(t_{n}-t_{n-1}\right)}, t_{n-1}\right),
\end{array}
$$

where

$$
\mathcal{F}=\exp \left[-\int_{t_{0}}^{t}\left(\frac{k^{2}}{3 H}+\frac{V^{\prime \prime}}{3 H V}-\frac{V^{\prime 2}}{3 H V^{2}}\right) d t^{\prime}\right]
$$

and

$$
\mathcal{G}=-\frac{2 V^{\prime \prime \prime}}{3 H V}+\frac{2 V^{\prime} V^{\prime \prime}}{H V^{2}}-\frac{4 V^{\prime 3}}{3 H V^{3}} .
$$

The second term on the right hand side of equations (54) and (55) are memory terms which give the concept of freeze-out. When $k \geq k_{F}$ this term damps away in a Hubble time and when $k \leq k_{F}$ its effect is not negligible. In this regard, the freeze-out momentum $k_{F}$ is expressed by the following condition

$$
\frac{k_{F}^{2}}{3 H^{2}}+\frac{V^{\prime \prime}}{3 H^{2} V}-\frac{V^{\prime 2}}{3 H^{2} V^{2}}=1
$$

So, the freeze-out momentum $k_{F}$ is given by

$$
k_{F}=\sqrt{\frac{3 H^{2} V^{2}-V^{\prime \prime} V+V^{\prime 2}}{V^{2}}}
$$

Now, we compute the three-point correlation function of the tachyon fluctuations at large scale, at the time about 50 e-folds before the end of inflation, by using equations (54) and (55). The result is as follows

$$
\begin{aligned}
& \left\langle\delta \phi\left(\mathbf{k}_{1}, t\right) \delta \phi\left(\mathbf{k}_{2}, t\right) \delta \phi\left(\mathbf{k}_{3}, t\right)\right\rangle=\mathcal{F}\left(k_{3}, t-t_{50}-\frac{1}{H}\right) \\
& \int_{t_{50}-\frac{1}{H}}^{t_{50}} d t^{\prime} \mathcal{F}^{-1}\left(k_{3}, t^{\prime}-t_{50}-\frac{1}{H}\right) \mathcal{G}\left(k_{3}, t^{\prime}\right) \\
& {\left[\int \frac{d p^{3}}{(2 \pi)^{3}}\left\langle\delta \phi^{(1)}\left(\mathbf{k}_{1}, t_{1}\right) \delta \phi^{(1)}\left(\mathbf{p}, t^{\prime}\right)\right\rangle\right.} \\
& \left.\left\langle\delta \phi^{(1)}\left(\mathbf{k}_{2}, t_{2}\right) \delta \phi^{(1)}\left(\mathbf{k}_{3}-\mathbf{p}, t^{\prime}\right)\right\rangle\right]+\mathcal{F}\left(k_{3}, t-t_{60}-\frac{1}{H}\right) \\
& \left\langle\delta \phi^{(1)}\left(\mathbf{k}_{1}, t_{60}\right) \delta \phi^{(1)}\left(\mathbf{k}_{2}, t_{60}\right) \delta \phi^{(1)}\left(\mathbf{k}_{3} e^{-1}, t_{60}-\frac{1}{H}\right)\right\rangle \\
& +\left(\mathbf{k}_{1} \leftrightarrow \mathbf{k}_{3}\right)+\left(\mathbf{k}_{2} \leftrightarrow \mathbf{k}_{3}\right)
\end{aligned}
$$

On the scale $k<k_{F}$, we can take $\mathcal{F}$ about unity and $\mathcal{G}$ as a constant and so we have 

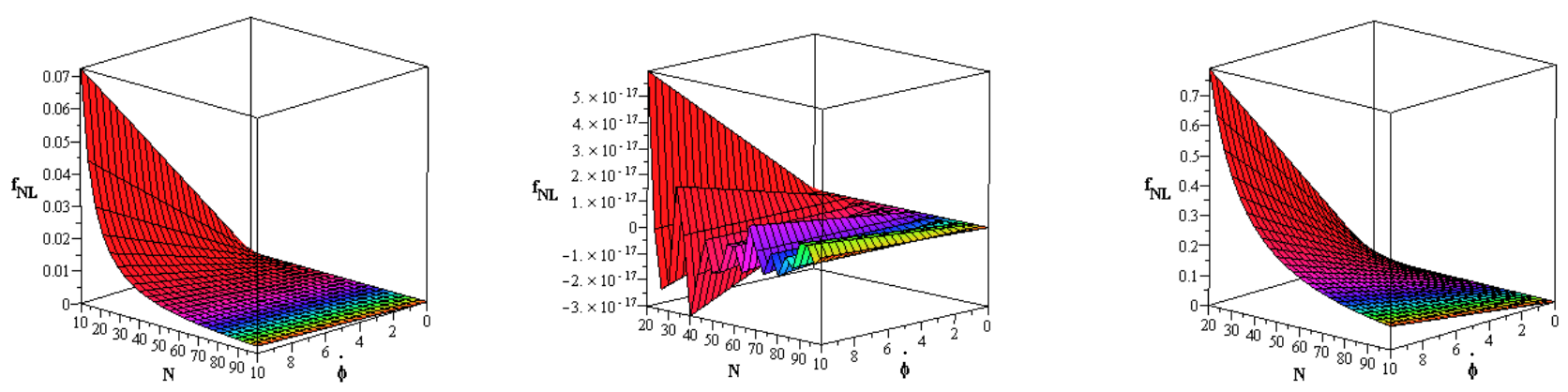

FIG. 7. Evolution of the non-gaussianity with respect to $N$ and $\dot{\phi}$ for a quadratic potential (left panel), an exponential potential (meddle panel) and an intermediate potential (right panel) in minimal coupling setup.

$$
\begin{aligned}
\left\langle\delta \phi\left(\mathbf{k}_{1}, t\right) \delta \phi\left(\mathbf{k}_{2}, t\right) \delta \phi\left(\mathbf{k}_{3}, t\right)\right\rangle & \approx \mathcal{G}\left(k_{F}, t_{F}\right) \frac{1}{H} \ln \left(\frac{k_{F}}{H}\right) \times \\
& {\left[\int \frac{d^{3} p}{(2 \pi)^{3}}\left\langle\delta \phi^{(1)}\left(\mathbf{k}_{1}, t_{1}\right) \delta \phi^{(1)}\left(\mathbf{p}, t^{\prime}\right)\right\rangle\left\langle\delta \phi^{(1)}\left(\mathbf{k}_{2}, t_{2}\right) \delta \phi^{(1)}\left(\mathbf{k}_{3}-\mathbf{p}, t^{\prime}\right)\right\rangle+\left(\mathbf{k}_{1} \leftrightarrow \mathbf{k}_{3}\right)+\left(\mathbf{k}_{2} \leftrightarrow \mathbf{k}_{3}\right)\right] }
\end{aligned}
$$

The bispectrum for the single field inflation models in the

slow-roll limit is given by the following expression [8]

$$
\left\langle\Phi\left(\mathbf{k}_{1}\right) \Phi\left(\mathbf{k}_{2}\right) \Phi\left(\mathbf{k}_{3}\right)\right\rangle=2 f_{N L}(2 \pi)^{3} \delta^{3}\left(\mathbf{k}_{1}+\mathbf{k}_{2}+\mathbf{k}_{3}\right)\left[P_{\Phi}\left(\mathbf{k}_{1}\right)+P_{\Phi}\left(\mathbf{k}_{2}\right)+\text { permutations }\right] .
$$

On the other hand, the gravitational field potential is given by [7, 60]

$$
\Phi(\mathbf{k})=-\frac{3}{2} \frac{H}{\dot{\phi}} \delta \phi(\mathbf{k})
$$

So, from equations (61), (62) and (63) we obtain

$f_{N L}=-\frac{2}{3} \frac{\dot{\phi}}{H}\left[\frac{1}{H} \ln \left(\frac{k_{F}}{H}\right)\left(\frac{2 V^{\prime \prime \prime}}{3 H V}-\frac{2 V^{\prime} V^{\prime \prime}}{H V^{2}}+\frac{4 V^{\prime 3}}{3 H V^{3}}\right)\right]$

In figure 7 we have plotted the behavior of $f_{N L}$ versus $\dot{\phi}$ and $N$ for three types of potentials: quadratic, exponential and intermediate potential. As figure shows, both for quadratic and intermediate potentials, $f_{N L}$ is positive and decreases as the number of e-folds parameter increases. Note that for the intermediate potential we have set $l=0.4$. With an exponential potential the nongaussianity can be either positive or negative, depending on the values of $N$ and $\dot{\phi}$. Also, for this type of potential the value of non-gaussianity is so small.

\section{Cosmological dynamics and late time acceleration}

In this section, we study cosmological dynamics of the minimally coupled tachyon field model by using the dynamical system analysis and phase space trajectories of the model. The Friedmann equation in the presence of the tachyon field and ordinary components (matter and radiation) is given by

$$
H^{2}=\frac{\kappa^{2}}{3}\left[\frac{V(\phi)}{\sqrt{1-\dot{\phi}^{2}}}+\rho\right],
$$

where $\rho$ is the energy density of the ordinary components. In order to analyze the phase space of the model, we firstly introduce some new convenient and dimensionless variables which help us to translate the equations of the cosmological dynamics in the language of the autonomous dynamical system. In this regard, we define the following dimensionless parameters in our setup

$$
x=\dot{\phi}, \quad y=\frac{\kappa \sqrt{V}}{\sqrt{3} H}, \quad d=\frac{\kappa \sqrt{\rho}}{\sqrt{3} H}, \quad \alpha=\frac{V^{\prime}}{\kappa_{4} V^{\frac{3}{2}}},
$$


We can rewrite the Friedmann equation (65) in terms of these dimensionless parameters and obtain the following constraint on the model's parameter space

$$
1=\frac{y^{2}}{\sqrt{1-x^{2}}}+d^{2} .
$$

We note that, the constraint equation (67) allows us to express one of the dimensionless variables in terms of the others.

Now, we rewrite other important equations of the setup in terms of the new variables. The acceleration equation in terms of new variables takes the following form

$$
\frac{\dot{H}}{H^{2}}=-\frac{3}{2} \frac{y^{2} x^{2}}{\sqrt{1-x^{2}}}-\frac{3}{2} d^{2}(1+\omega) .
$$

Also, the scalar field's equation of motion (8) is given by the following expression

$$
\frac{\ddot{\phi}}{H}=-3 x\left(1-x^{2}\right)-\sqrt{3} y \alpha\left(1-x^{2}\right) .
$$

We can reformulate the effective equation of state parameter in terms of the dimensionless parameters as

$$
\omega_{e f f}=-y^{2} \sqrt{1-x^{2}}+\omega\left(1-\frac{y^{2}}{\sqrt{1-x^{2}}}\right) .
$$

In the next step, we introduce a new time variable $\tau \equiv \ln a$, and obtain the following autonomous system of equations in our setup

$$
\frac{d x}{d \tau}=-3 x\left(1-x^{2}\right)-\sqrt{3} y \alpha\left(1-x^{2}\right),
$$

and

$$
\frac{d y}{d \tau}=\frac{\sqrt{3}}{2} y^{2} \alpha x-y\left(1-\frac{3 y^{2} x^{2}}{\sqrt{1-x^{2}}}\right) .
$$

We choose the inverse power law potential $V(\phi)=\frac{1}{\kappa_{4}^{2} \phi^{2}}$ (see [15] for reliability of this choice), which leads to a constant $\alpha$ and so $\frac{d \alpha}{d \tau}=0$.

Now, we analyze the cosmological evolution of this setup in the dynamical system approach. To this end, we should find the fixed (or critical) points in the phase space of the model. Fixed points are defined as the points where the autonomous equations (71) and (72) are vanishing. In the minimally coupled tachyon field setup, there is 5 critical points; $M, N, O, P$ and $Q$. The properties of these critical points are summarized in table III The points $P$ and $Q$ are effectively ordinary components dominated solutions. Their eigenvalues are positive, so these critical points are repeller nodes. These solutions can be corresponding to the early time radiation or matter domination era in the history of the universe. The point $O$ is effectively ordinary components dominated. This point is an unstable saddle point, since one of its

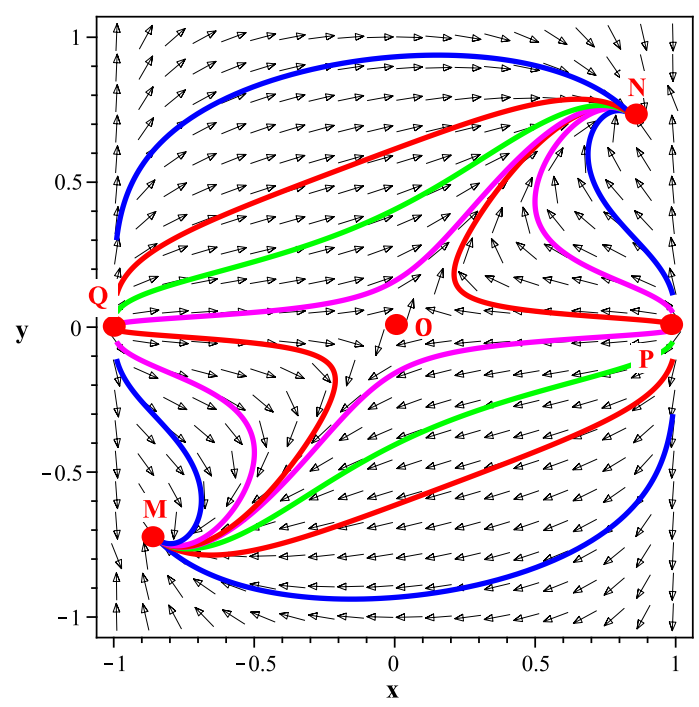

FIG. 8. The phase space trajectories of the minimally coupled tachyon field model. The critical point $O$ is an effectively ordinary components (matter or radiation) dominated solution and is an unstable saddle point. Points $P$ and $Q$ are unstable too. The points $N$ and $M$ are effectively tachyon field dominated solutions and are stable critical points. There is no accelerated phase of expansion.

eigenvalues is positive and the other one is negative. If the universe during its evolution reaches this point, it doesn't remain there and evolves to another state. Note that, although $P, Q$ and $O$, all are effectively ordinary components dominated phases, there is a difference between them. At the point $O$, other components of the universe (the kinetic and potential terms of the tachyon field) have no contribution. While, at the points $P$ and $Q$ the kinetic term of the tachyon field has significant contribution. The points $M$ and $N$ are stable critical points, meaning that if the universe reaches these states, remains there forever. However, in this case the universe expansion is not accelerating (as the effective equation of state parameter shows). The trajectories of the phase space of the minimally coupled tachyon field model, for $\omega=0$, is shown in figure 8 . Note that, the location of the fixed points are independent of $\omega$. For both $\omega=0$ and $\omega=\frac{1}{3}$, the trajectories of the phase space are the same but the eigenvalues and effective equation of state parameters are different.

Figure 9 shows the evolution of the effective equation of state parameter with respect to the cosmic time. As we see form this figure, the effective equation of state parameter of the model tends to the value of about -0.286 . So there is no possibility to realize the accelerating phase of the universe expansion in a model with a minimally coupled tachyon field. 
TABLE III. Location, Eigenvalues and Dynamical Characters of the Critical Points.

\begin{tabular}{ccccc}
\hline \hline Point & $(x, y)$ & eigenvalues & $\omega_{\text {eff }}$ & stability \\
\hline$N$ & $(0.84480,0.73159)$ & $-0.8594-3 \omega,-1.9296$ & -0.2863887966 & stable \\
& & & $-0.000264873 \omega$ & $\omega$ \\
\hline$O$ & $(0,0)$ & $-3, \frac{3}{2}+\frac{3}{2} \omega$ & -0.2863887966 & unstable \\
\hline$M$ & $(-0.84480,-0.73159)$ & $-0.8594-3 \omega,-1.9296$ & $-0.000264873 \omega$ & stable \\
\hline$P$ & $(1,0)$ & & $\omega$ & unstable \\
\hline$Q$ & $(-1,0)$ & $6, \frac{3}{2}+\frac{3}{2} \omega$ & $\omega$ & unstable \\
\hline \hline
\end{tabular}
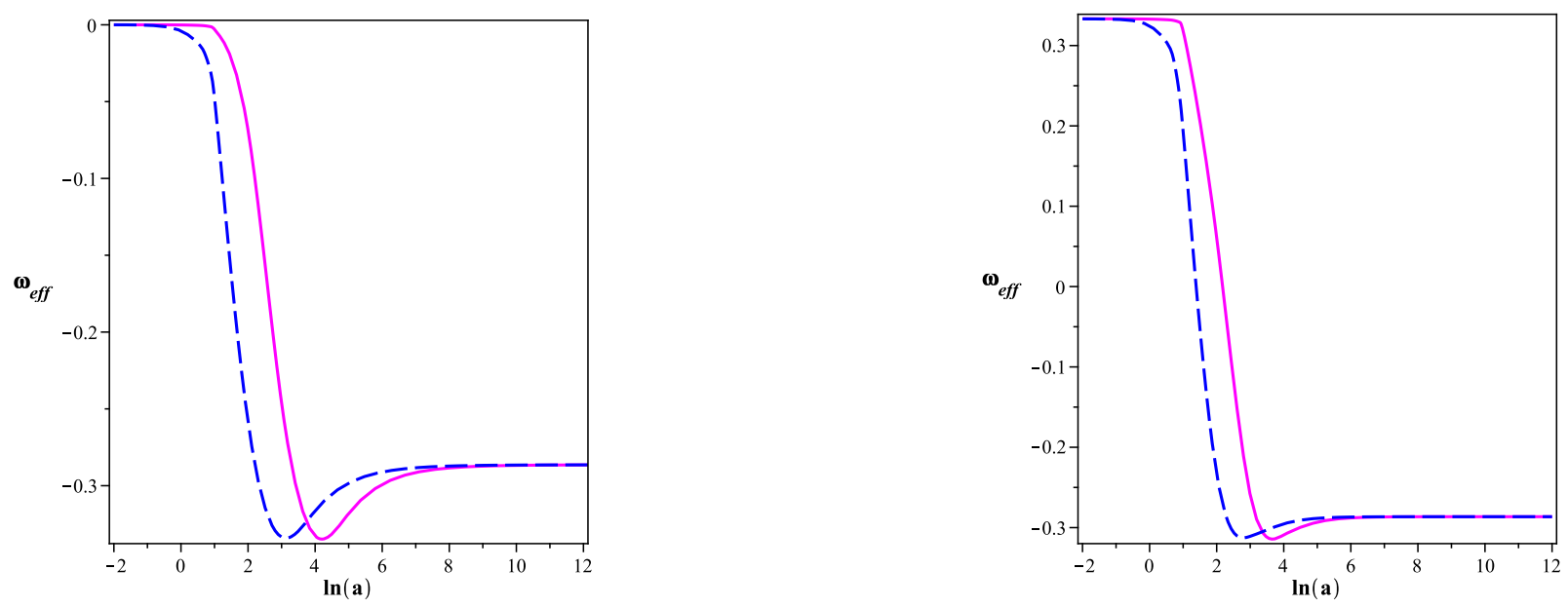

FIG. 9. Behavior of the effective equation of state parameter versus the cosmic time. The left panel is corresponding to $\omega=0$ (dust) and the right panel is corresponding to $\omega=\frac{1}{3}$ (radiation). $\omega_{\text {eff }}$ evolves and tends to the value -0.286 . These figures have been plotted with $x(0)=0.99$ and $y(0)=0.01$ (the magenta curve) and $x(0)=0.75$ and $y(0)=0.05$ (the blue curve).

\section{E. Statefinder Diagnostic}

In 2003 Sahni et al. have proposed a diagnostic proposal by introducing a new pair of parameters $\{r, s\}$, called statefinder parameters, in order to distinguish between different types of dark energy models [76]. These parameters are defined as

$$
\begin{gathered}
r=\frac{\dddot{a}}{a H^{3}}=\frac{\ddot{H}}{H^{3}}-3 q-2, \\
s=\frac{r-1}{3\left(q-\frac{1}{2}\right)} .
\end{gathered}
$$

Since statefinder parameters depend on the scale factor and therefore, on the metric describing the space-time, it is a "geometrical" diagnostic. By using the statefinder parameters, we can study the expansion history of the universe through higher derivatives of the scale factor (that is, $\dddot{a}$ ). The $\Lambda \mathrm{CDM}$ scenario corresponds to a fixed point in the $r$-s diagram with $\{r, s\}_{\Lambda C D M}=\{1,0\}$. By plotting the trajectories in the $r$-s phase diagram, the distance of the model from $\Lambda \mathrm{CDM}$ can be probed [77].
We can rewrite the equation (73) as

$$
r=\frac{d}{d \tau}\left(\frac{\dot{H}}{H^{2}}\right)+2\left(\frac{\dot{H}}{H^{2}}\right)^{2}+3\left(\frac{\dot{H}}{H^{2}}\right)+1,
$$

which in our setup with a minimally coupled tachyon field takes the following form

$$
\begin{gathered}
r=\frac{-3 y y^{\prime} x^{2}-3 y^{2} x x^{\prime}}{\sqrt{1-x^{2}}}+\frac{3}{2} \frac{y^{2} x^{3} x^{\prime}}{\left(1-x^{2}\right)^{\frac{3}{2}}}-3 d d^{\prime}(1+\omega) \\
+\frac{9}{2}\left(\frac{y^{2} x^{2}}{\sqrt{1-x^{2}}}+d^{2}(1+\omega)\right)^{2} \\
\quad-\frac{9}{2}\left(\frac{y^{2} x^{2}}{\sqrt{1-x^{2}}}+d^{2}\left(1+\omega_{m}\right)\right)+1
\end{gathered}
$$

The other parameter, $s$, can be obtained by substituting equation (76) in equation (74) that we avoid to rewrite it here.

Now, we proceed to numerical analysis of the statefinder diagnostic. Figure 10 shows the trajectories of $\{r, q\}$ phase plane for two initial values of the parameters. The left panel is $\{r, q\}$ diagram with $\omega=0$ (corresponding to the dust) and the right panel is trajectories of $\{r, q\}$ phase plane with $\omega=\frac{1}{3}$ (corresponding to the radiation). For both panels, independent of the 

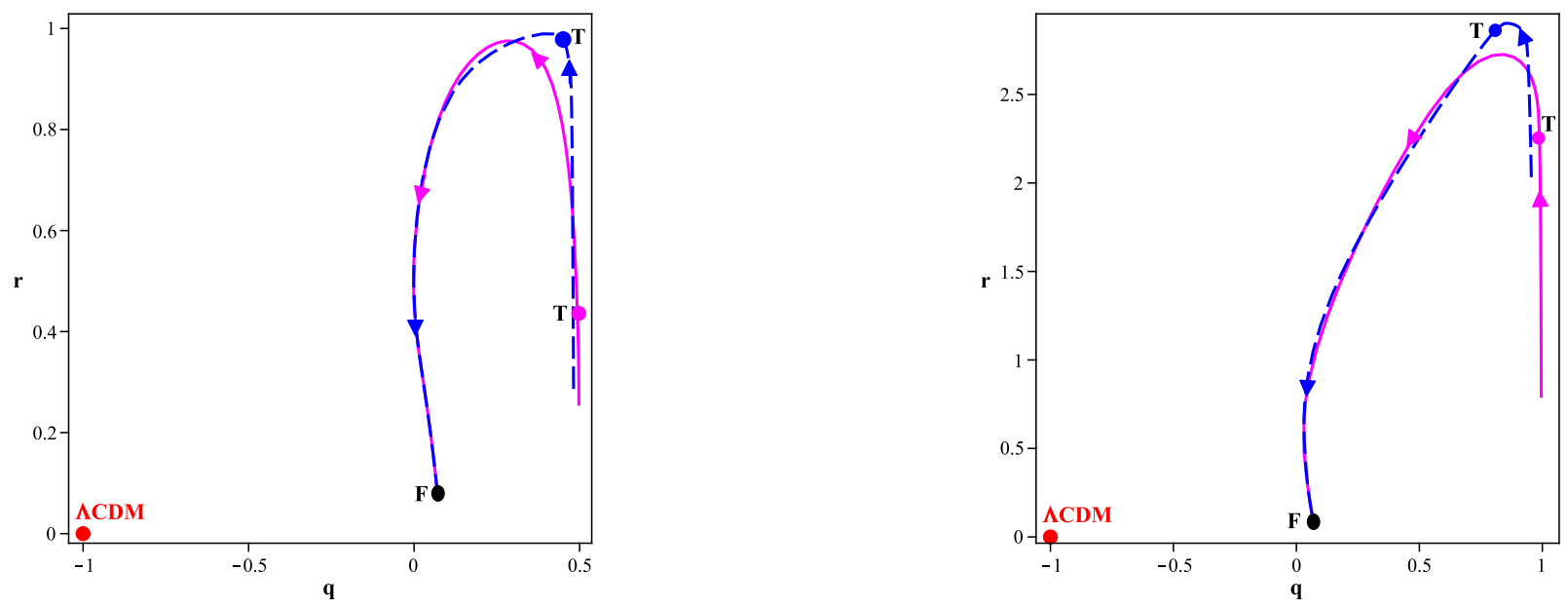

FIG. 10. Trajectories in $\{r, q\}$ phase plan with $\omega=0$ (left panel, corresponding to the dust) and $\omega=\frac{1}{3}$ (right panel, corresponding to the radiation). The initial conditions are $x(0)=0.99$ and $y(0)=0.01$ for magenta trajectory and $x(0)=0.75$ and $y(0)=0.05$ for blue trajectory. The magenta and blue highlighted dots (specified by $T$ ) are current values of $\{r, q\}$ in the model. The black dot (specified by $F$ ) is the stable state of $\{r, q\}$ in the future. Also the red dot (remarked by $\Lambda$ CDM) is the value of statefinder $\{r, q\}$ in a $\Lambda$ CDM scenario.
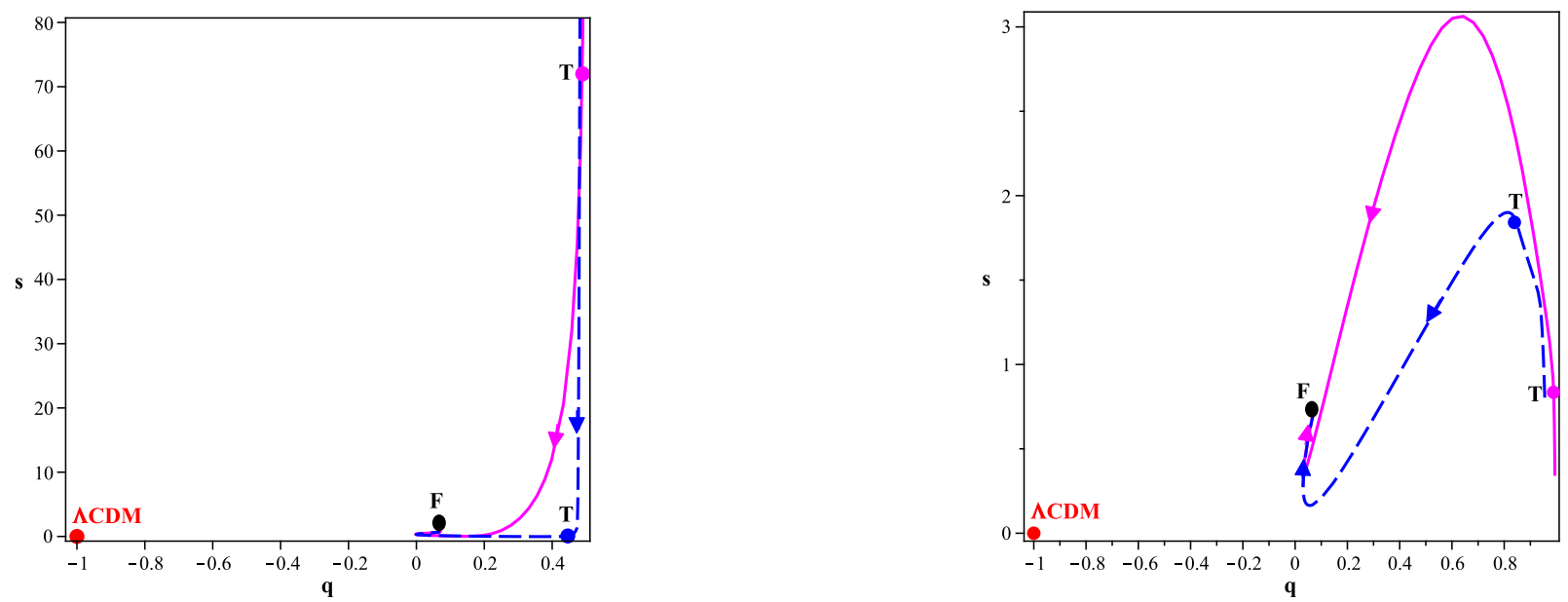

FIG. 11. Trajectories in $\{s, q\}$ phase plan with $\omega=0$ (left panel) and $\omega=\frac{1}{3}$ (right panel). The initial conditions are $x(0)=0.99$ and $y(0)=0.01$ for magenta trajectory and $x(0)=0.75$ and $y(0)=0.05$ for blue trajectory. The magenta and blue highlighted dots (specified by $T$ ) are current values of $\{s, q\}$ in the model. The black dot (specified by $F$ ) is the stable state of $\{s, q\}$ in the future. Also the red dot (remarked by $\Lambda \mathrm{CDM})$ is the value of statefinder $\{s, q\}$ in a $\Lambda$ CDM scenario.

initial conditions, the trajectories evolve to a fixed point in the future. The magenta and blue highlighted dots show the current values of $r$ and $q .\{s, q\}$ phase plan for both $\omega=0$ (left panel) and $\omega=\frac{1}{3}$ (right panel) is shown in figure 11] Here also, independent of the initial conditions, the trajectories tend to a fixed point in the future. Finally, we can see the $\{r, s\}$ diagram in figure 12. As the evolution of $q$ shows, this model can not explain the late time speed-up expansion of the universe. There is no stable point which could explain the late time acceleration.

After a detailed study of a minimally coupled tachyon field and its cosmological dynamics, now we extend our analysis to the more general case of a non-minimally cou- pled tachyon field. 

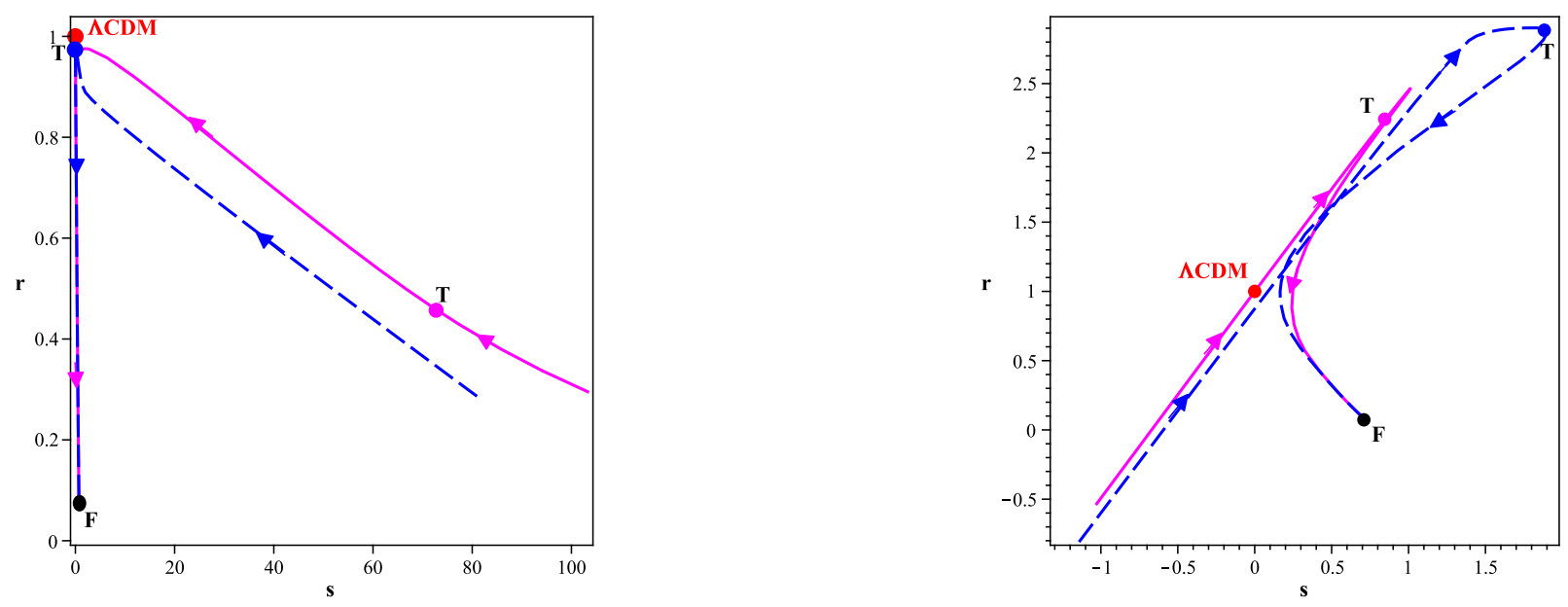

FIG. 12. The trajectories in $\{r, s\}$ phase plan with $\omega=0$ (the left panel, corresponding to dust) and $\omega=\frac{1}{3}$ (the right panel, corresponding to radiation). The initial conditions are $x(0)=0.99$ and $y(0)=0.01$ for magenta trajectory and $x(0)=0.75$ and $y(0)=0.05$ for blue trajectory. The magenta and blue bold dots (specified by $T$ ) are current values of $\{r, s\}$ in the model. The black dot (specified by $F$ ) is the stable state of $\{r, s\}$ in the future. Also the red dot (remarked by $\Lambda$ CDM) is the value of statefinder $\{r, s\}$ in a $\Lambda$ CDM scenario.

\section{NON-MINIMALLY COUPLED TACHYON FIELD}

\section{A. Inflation}

The 4-dimensional action for a non-minimally coupled tachyon field is given by the following expression

$$
S=\int \sqrt{-g}\left[\frac{1}{\kappa^{2}} R-\frac{1}{2} f(\phi) R-V(\phi) \sqrt{1-\partial^{\mu} \phi \partial_{\mu} \phi}\right] d^{4} x,
$$

where $f(\phi)$ shows an explicit non-minimal coupling of the scalar field with the Ricci scalar. Einstein's field equations calculated from action (77) are given by

$$
\left(1-\kappa^{2} f(\phi)\right) G_{\mu \nu}=\kappa^{2} T_{\mu \nu}
$$

where $T_{\mu \nu}$ is the energy momentum tensor of a nonminimally coupled tachyon field, given by

$$
\begin{gathered}
T_{\mu \nu}=-\frac{V(\phi)}{\sqrt{1-\partial_{\alpha} \phi \partial^{\alpha} \phi}} \partial_{\mu} \phi \partial_{\nu} \phi+\left(g_{\mu \nu} \square-\nabla_{\mu} \nabla_{\nu}\right) f(\phi) \\
+g_{\mu \nu}\left(-V(\phi) \sqrt{1-\partial_{\alpha} \phi \partial^{\alpha} \phi}\right)
\end{gathered}
$$

The energy-momentum tensor (79) leads to the following energy density and pressure

$$
\rho_{\phi}=\frac{V(\phi)}{\sqrt{1-\dot{\phi}^{2}}}+3 f^{\prime} H \dot{\phi}
$$

and

$$
p_{\phi}=-V(\phi) \sqrt{1-\dot{\phi}^{2}}-f^{\prime} \ddot{\phi}-2 f^{\prime} H \dot{\phi}-2 f^{\prime \prime} \dot{\phi}^{2} .
$$

By using the line element (6), we find the Friedmann equation of this model as follows

$$
H^{2}=\frac{\kappa^{2}}{3-3 \kappa^{2} f}\left[\frac{V(\phi)}{\sqrt{1-\dot{\phi}^{2}}}+3 f^{\prime} H \dot{\phi}\right] \text {. }
$$

By varying the action (77) with respect to the scalar field, the equation of motion of the non-minimally coupled tachyon field is obtained as

$$
\frac{\ddot{\phi}}{1-\dot{\phi}^{2}}+3 H \dot{\phi}+\frac{V^{\prime}}{V}+\frac{\sqrt{1-\dot{\phi}^{2}}}{2 V} f^{\prime} R=0
$$

where, as before, a prime denotes derivative with respect to the tachyon field and a dot refers to derivative with respect to the time.

In the presence of a non-minimally coupled tachyon field, the energy conservation equation takes the following form

$$
\dot{\rho}_{\phi}+3 H\left(\rho_{\phi}+p_{\phi}\right)=-3 f^{\prime} H^{2} \dot{\phi} .
$$

In contrast to equation (9), the right hand side of equation (84) is not zero due to the presence of the nonminimal coupling between the tachyon field and the Ricci scalar.

Now, we apply the slow-roll approximation to the main equations of the setup. The energy density and the equation of motion of the tachyon field, in the slow-roll limit, are given respectively as

$$
\rho_{\phi}=V(\phi)+3 f^{\prime} H \dot{\phi},
$$

and

$$
3 H \dot{\phi}+\frac{V^{\prime}}{V}+\frac{f^{\prime} R}{2 V}=0 .
$$


Also, the Friedmann equation takes the following form

$$
H^{2}=\frac{\kappa^{2}}{1-\kappa^{2} f}\left[-\frac{f^{\prime 2} R}{V}+\frac{V}{3}-\frac{f^{\prime} V^{\prime}}{3 V}\right] .
$$

$$
\begin{aligned}
\epsilon=\frac{1}{2 \kappa^{2}} \frac{V^{\prime 2}}{V^{2}} \frac{1-\kappa^{2} f}{\left[-\frac{f^{\prime 2} R}{2 V}+V-\frac{f^{\prime} V^{\prime}}{V}\right]^{2}}\left[\frac{f^{\prime} R}{2 V^{\prime 2}}+\frac{1}{V^{\prime}}\right] \times \\
\quad\left[-f^{\prime} f^{\prime \prime} R+\frac{f^{\prime 2} R V^{\prime}}{2 V}+V^{\prime} V-f^{\prime \prime} V-f^{\prime} V^{\prime \prime}+\frac{f^{\prime 2} V^{\prime 2}}{V}+\frac{\kappa^{2} f^{\prime}}{1-\kappa^{2} f}\left[V^{2}-\frac{f^{\prime 2} R}{2}-f^{\prime} V^{\prime}\right]\right],
\end{aligned}
$$

$$
\eta=\frac{\kappa^{2} f^{\prime}}{3 H^{2}}\left(\frac{f^{\prime} R}{2 V}+\frac{V^{\prime}}{V}\right)-\frac{1-\kappa^{2} f}{3 X}\left[3 X-V^{\prime}-3 f^{\prime} H^{2}\right]-\frac{\dot{B}}{\frac{\kappa^{2} X}{3 H\left(1-\kappa^{2} f\right)}\left(\frac{f^{\prime} R}{2 V}-\frac{V^{\prime}}{V}\right)},
$$

where $B$ and $X$ are defined as

$$
B=\frac{\kappa^{2}}{H}\left[X-f^{\prime} H^{2}\right],
$$

and

$$
\begin{aligned}
X=-\frac{f^{\prime} f^{\prime \prime} R}{3 V} & +\frac{f^{\prime 2} V^{\prime} R}{6 V^{2}}+\frac{V^{\prime}}{3} \\
& -\frac{f^{\prime \prime} V^{\prime}}{3 V}+\frac{f^{\prime 2} V^{\prime 2}}{3 V^{2}}-\frac{f^{\prime} V^{\prime \prime}}{3 V}+f^{\prime} H^{2} .
\end{aligned}
$$

The number of e-folds, which is defined by equation (17), in the slow-roll approximation is given by

$$
N \simeq \int_{\phi_{h c}}^{\phi_{f}}\left(\frac{3 V^{\prime}}{V}\right)\left(\frac{\kappa^{2}\left[\frac{2 V V^{\prime}}{3}-\frac{f^{\prime 2} V^{\prime} R}{3 V}-\frac{f^{\prime} V^{\prime 2}}{3 V}\right]}{\left(1-\kappa^{2} f\right)\left(-f^{\prime} R-2 V^{\prime}\right)}\right) d \phi .
$$

As the minimal case, in which follows we explore the scalar perturbation of the metric in order to study the spectrum of perturbations.

\section{B. Perturbations}

Similar to the minimal case, we use the perturbed metric (20) which is written in the longitudinal gauge. With this perturbed metric, we obtain the following perturbed Einstein's field equations

$$
\begin{gathered}
-3 H(H \Phi+\dot{\Psi})-\frac{k^{2}}{a^{2}}=\frac{\kappa_{4}^{2}}{2} \delta \rho_{e f f}, \\
\ddot{\Psi}+3 H(H \Phi+\dot{\Psi})+H \dot{\Phi}+2 \dot{H} \Phi+\frac{1}{3 a^{2}} k^{2}(\Phi-\Psi)=\frac{\kappa_{4}^{2}}{2} \delta p_{\text {eff }},
\end{gathered}
$$

$$
\begin{aligned}
& \dot{\Psi}+H \Phi= \\
& -\frac{\kappa^{2} V(\phi)}{\sqrt{1-\dot{\phi}^{2}}} \frac{\dot{\phi} \delta \phi}{2\left(1-\kappa^{2} f\right)}+\frac{\kappa^{2} \int\left(\delta T_{i}^{0}\right)^{(n m c)} d x^{i}}{2\left(1-\kappa^{2} f\right)},
\end{aligned}
$$

$$
\Psi-\Phi=-\frac{\kappa^{2} \delta f}{1-\kappa^{2} f}
$$

By comparing equations (93)-(96) with equations (21)(24), we can see the effects of the non-minimal coupling between tachyon field and Ricci scalar in perturbed field equations. In equations (93) and (94), $\rho_{\text {eff }}$ and $p_{\text {eff }}$ are the effective energy density and pressure which contain the effects of non-minimal coupling. We obtain these effective parameters from the standard Friedmann equation $H^{2}=\frac{\kappa_{4}^{2}}{3} \rho_{\text {eff }}$ and effective conservation equation $\dot{\rho}_{\text {eff }}+3 H\left(\rho_{\text {eff }}+p_{\text {eff }}\right)=0$ as follows

$$
\rho_{e f f}=\frac{\rho_{\phi}}{1-\kappa^{2} f},
$$

and

$$
p_{e f f}=\frac{p_{\phi}}{1-\kappa^{2} f} .
$$

where $\rho_{\phi}$ and $p_{\phi}$ are defined by equations (80) and (81) respectively. By perturbing the equations (97) and (98), we find

$$
\begin{aligned}
\delta \rho_{e f f}= & \frac{1}{1-\kappa^{2} f}\left[\frac{V^{\prime} \delta \phi}{\sqrt{1-\dot{\phi}^{2}}}\right. \\
& \left.-V \frac{\dot{\phi} \delta \dot{\phi}+\dot{\phi}^{2} \Phi}{\left(1-\dot{\phi}^{2}\right)^{\frac{3}{2}}}+\frac{\kappa^{2} \rho_{\phi} \delta f}{1-\kappa^{2} f}+\delta \rho^{(n m c)}\right],
\end{aligned}
$$


and

$$
\begin{aligned}
\delta p= & \frac{1}{1-\kappa^{2} f}\left[-V^{\prime} \sqrt{1-\dot{\phi}^{2}} \delta \phi\right. \\
& \left.-V \frac{\dot{\phi} \delta \dot{\phi}+\dot{\phi}^{2} \Phi}{\sqrt{1-\dot{\phi}^{2}}}+\frac{\kappa^{2} p_{\phi} \delta f}{1-\kappa^{2} f}+\delta p^{(n m c)}\right] .
\end{aligned}
$$

$\delta \rho^{(n m c)}$ and $\delta p^{(n m c)}$ are the perturbed energy density and perturbed pressure corresponding to the non-minimal sector of the energy-momentum tensor which is given by the following expression

$$
T_{\mu \nu}^{(n m c)}=\left(g_{\mu \nu} \square-\nabla_{\mu} \nabla_{\nu}\right) f .
$$

By perturbing the $(0,0)$ and $(i, i)$ (with $i=1, \ldots, 3)$ components of the equation (101), we obtain the perturbed non-minimal sector of the energy density and pressure respectively as follows

$$
\delta \rho^{(n m c)}=2 \Phi\left[\left(\square+\nabla_{0} \nabla^{0}\right) f\right]+\left[-2 \Phi \square f-\left(\square+\nabla_{0} \nabla^{0}\right) \delta f\right],
$$

and

$$
\begin{array}{r}
\delta p^{(n m c)}=\delta_{j}^{b}\left[\delta g^{a j}\left(g_{a b} \square-\nabla_{a} \nabla_{b}\right) f+g^{a j} \delta g_{a b} \square f\right. \\
\left.+g^{a j}\left(g_{a b} \square-\nabla_{a} \nabla_{b}\right) \delta f\right] .
\end{array}
$$

The effects of the non-minimal coupling is seen by the presence of $\left(\delta T_{i}^{0}\right)^{(n m c)}$ in equation (95) and also by the presence of parameter $f$ in equations (95) and (96). Especially, the non-minimal coupling of the tachyon field and the Ricci scalar causes that the two metric perturbations ( $\Psi$ and $\Phi)$ are not equal anymore. The equation (96) shows this property clearly.

Variation of the scalar field's equation of motion in the presence of the non-minimal coupling is given by the following expression

$$
\begin{aligned}
\delta \ddot{\phi}+3 H \delta \dot{\phi}+2 \dot{\phi} \ddot{\phi} \frac{\delta \dot{\phi}+\dot{\phi}^{2} \Phi}{1-\dot{\phi}^{2}}+\left[\frac{\dot{\phi} \delta \dot{\phi}+\ddot{\phi}^{2}}{2 V \sqrt{1-\dot{\phi}^{2}}}-\frac{V^{\prime} \delta \phi \sqrt{1-\dot{\phi}^{2}}}{2 V}\right] f^{\prime} R+\frac{\sqrt{1-\dot{\phi}^{2}}}{2 V} f^{\prime \prime} R \delta \phi \\
+\frac{\sqrt{1-\dot{\phi}^{2}}}{V} f\left[\left(\frac{k a^{2}}{a^{2}}-3 \dot{H}\right) \Phi-\frac{2 k^{2}}{a^{2}} \Psi-3\left(\ddot{\Psi}+4 H \dot{\Psi}+H \dot{\Phi}+\dot{H} \Phi+4 H^{2} \Phi\right)\right] \\
=\dot{\phi}(\dot{\Phi}+3 \dot{\Psi})+\left(6 H \dot{\phi}^{3}-\frac{2 V^{\prime}}{V}\left(1-\dot{\phi}^{2}\right)\right) \Phi+\left(1-\dot{\phi}^{2}\right) \delta \phi\left(\frac{V^{\prime \prime}}{V}-\frac{V^{\prime 2}}{V^{2}}\right) .
\end{aligned}
$$

If we set $f=0$, the above perturbed scalar field's equation of motion simplifies to the equation (27).

Now, we study variation of the curvature perturbation on a uniform-density hypersurface. In this regard, we rewrite the gauge-invariant primordial curvature perturbation $\zeta$, given by equation (28), as follows

$$
\zeta=\Psi-\frac{H}{\dot{\rho}_{e f f}} \delta \rho_{e f f} .
$$

From this equation, the time evolution of the primordial curvature perturbation is given by the following equation

$$
\dot{\zeta}=H\left(\frac{\delta p_{\text {nad }}}{\rho_{\text {eff }}+p_{\text {eff }}}\right)
$$

Similar to the minimal coupling case, we split the pressure perturbations into adiabatic and non-adiabatic parts as follows

$$
\delta p_{e f f}=c_{s}^{2} \delta \rho_{e f f}+\dot{p}_{e f f} \Gamma .
$$

In equation (107), the non-adiabatic part is $\delta p_{\text {nad }}=$ $\dot{p}_{e f f} \Gamma$. In the presence of the non-minimal coupling between the tachyon field and Ricci scalar, $\delta p_{\text {nad }}$ is not zero anymore. From equation (107), we can find the $\delta p_{\text {nad }}$ as follows 


$$
\begin{aligned}
\delta p_{n a d}=\frac{2}{\kappa^{2}\left(1-\kappa^{2} f\right)} & {\left[1-\dot{\phi}^{2}-\frac{\left(-V^{\prime} \dot{\phi} \sqrt{1-\dot{\phi}^{2}}+\frac{V \dot{\phi} \ddot{\phi}}{\sqrt{1-\dot{\phi}^{2}}}++\dot{p}_{n m c}\right)\left(1-\kappa^{2} f\right)+p \kappa^{2} f^{\prime} \dot{\phi}}{\left(-3 H(\rho+p)-3 f^{\prime} H^{2} \dot{\phi}\right)\left(1-\kappa^{2} f\right)+\rho \kappa^{2} f^{\prime} \dot{\phi}}\right] } \\
\times & {\left[-3 H(H \Phi+\dot{\Psi})-\frac{k^{2}}{a^{2}}\right]-\frac{1}{1-\kappa^{2} f}\left[2 V^{\prime} \delta \phi \sqrt{1-\dot{\phi}^{2}}+\delta \rho_{n m c}\left(1-\dot{\phi}^{2}\right)-\delta p_{n m c}\right] . }
\end{aligned}
$$

This non-vanishing, non-adiabatic pressure, leads to the non-vanishing time evolution of the primordial curvature perturbation as follows

$$
\begin{aligned}
\dot{\zeta}=-H\left[2 V^{\prime} \delta \phi \sqrt{1-\dot{\phi}^{2}}+\delta \rho_{n m c}\left(1-\dot{\phi}^{2}\right)-\delta p_{n m c}\right]+2\left[-3 H^{2}(H \Phi+\dot{\Psi})-H \frac{k^{2}}{a^{2}}\right] \times \\
{\left[\frac{\left(1-\dot{\phi}^{2}\right)^{\frac{3}{2}}}{\kappa^{2} V^{\prime} \dot{\phi}^{2}}+\frac{\left(V^{\prime} \dot{\phi} \sqrt{1-\dot{\phi}^{2}}-\frac{V \dot{\phi} \ddot{\phi}}{\sqrt{1-\dot{\phi}^{2}}}-\dot{p}_{n m c}\right)\left(1-\kappa^{2} f\right)\left(\sqrt{1-\dot{\phi}^{2}}\right)-p \kappa^{2} f^{\prime} \dot{\phi} \sqrt{1-\dot{\phi}^{2}}}{\kappa^{2} V^{\prime} \dot{\phi}^{2}\left(-3 H(\rho+p)-3 f^{\prime} H^{2} \dot{\phi}\right)\left(1-\kappa^{2} f\right)+\rho \kappa^{4} f^{\prime} V^{\prime} \dot{\phi}^{3}}\right] . }
\end{aligned}
$$

We see that, the non-minimal coupling behaves as a second field in the theory and causes non-adiabatic perturbation. So, the primordial curvature perturbations attain an explicit time-dependence.

Now, in order to obtain scalar and tensorial perturbations in our model, we take into account the slow-roll approximation at the large scales, $k \ll a H$. This is because the scales of cosmological interest have spent most of their time far outside the Hubble radius and have reentered only relatively recently in the Universe history. In this scale, $\ddot{\Phi}, \ddot{\Psi}, \dot{\Phi}$ and $\dot{\Psi}$ are negligible (see [78, 79]). So, at large scale, the perturbed tachon field's equation of motion takes the following form

$$
\begin{array}{r}
3 H \delta \dot{\phi}+\left(\frac{f^{\prime \prime} R-V^{\prime} f^{\prime} R}{2 V}-\frac{V^{\prime \prime}}{V}+\frac{V^{\prime 2}}{V^{2}}\right) \delta \phi \\
=\left(-\frac{f^{\prime} R}{V}-\frac{2 V^{\prime}}{V}\right) \Phi
\end{array}
$$

Also, from equation (95) and by using the relation $\int \delta\left(T_{i}^{0}\right)_{n m c} d x^{i}=2 f(H \Phi+\dot{\Psi})$, we obtain

$$
\Phi=-\frac{\kappa^{2} V \dot{\phi} \delta \phi}{2 H\left(1-2 \kappa^{2} f\right) \sqrt{1-\dot{\phi}^{2}}} .
$$

So, equation (110) can be rewritten as follows

$$
\begin{aligned}
3 H \delta \dot{\phi}=( & \left.-\frac{f^{\prime} R}{V}-\frac{2 V^{\prime}}{V}\right)\left(\frac{\kappa^{2} \dot{\phi} \delta \phi}{2 H\left(1-2 \kappa^{2} f\right) \sqrt{1-\dot{\phi}^{2}}}\right) \\
& -\left(\frac{f^{\prime \prime} R-V^{\prime} f^{\prime} R}{2 V}-\frac{V^{\prime \prime}}{V}+\frac{V^{\prime 2}}{V^{2}}\right) \delta \phi . \quad
\end{aligned}
$$

Here we introduce the function $\mathcal{F}$ as

$$
\mathcal{F} \equiv \frac{V \delta \varphi}{V^{\prime}}
$$

by which we rewrite equation (112) as follows

$$
\begin{array}{r}
\frac{\mathcal{F}^{\prime}}{\mathcal{F}}=\frac{\kappa^{2}\left(f^{\prime} R+2 V V^{\prime}\right)}{6 H^{2} V\left(1-\kappa^{2} f\right)} \\
-\frac{V^{\prime} f^{\prime} R-f^{\prime \prime} R-2 V^{\prime \prime}+\frac{2 V^{\prime 2}}{V}}{-f^{\prime} R-2 V^{\prime}}-\frac{V^{\prime \prime}}{V^{\prime}}+\frac{V^{\prime}}{V} .
\end{array}
$$

A solution of this equation is given by the following expression

$$
\mathcal{F}=\mathcal{C} \exp \left(\int \frac{\mathcal{F}^{\prime}}{\mathcal{F}} d \varphi\right)
$$

where $\mathcal{C}$ is an integration constant. So, from equation (113) we find

$$
\delta \phi=\frac{\mathcal{C} V^{\prime}}{V} \exp \left[\int\left(\frac{\kappa^{2}\left(f^{\prime} R+2 V V^{\prime}\right)}{6 H^{2} V\left(1-\kappa^{2} f\right)}-\frac{V^{\prime} f^{\prime} R-f^{\prime \prime} R-2 V^{\prime \prime}+\frac{2 V^{\prime 2}}{V}}{-f^{\prime} R-2 V^{\prime}}-\frac{V^{\prime \prime}}{V^{\prime}}+\frac{V^{\prime}}{V}\right) d \phi\right]
$$


So, the density perturbation amplitude is given by

$$
A_{s}^{2}=\frac{k^{3} \mathcal{C}}{2 \pi^{2}} \frac{V^{\prime 2}}{V^{2}} \exp \left[2 \int\left(\frac{\kappa^{2}\left(f^{\prime} R+2 V V^{\prime}\right)}{6 H^{2} V\left(1-\kappa^{2} f\right)}-\frac{V^{\prime} f^{\prime} R-f^{\prime \prime} R-2 V^{\prime \prime}+\frac{2 V^{\prime 2}}{V}}{-f^{\prime} R-2 V^{\prime}}-\frac{V^{\prime \prime}}{V^{\prime}}+\frac{V^{\prime}}{V}\right) d \phi\right] .
$$

By using this equation, the scalar spectral index (defined

by equation (37)) becomes

$$
n_{s}-1=\left[\frac{\kappa^{2}\left(f^{\prime} R+2 V V^{\prime}\right)}{3 H^{2} V\left(1-\kappa^{2} f\right)}-\frac{2 V^{\prime} f^{\prime} R-2 f^{\prime \prime} R-4 V^{\prime \prime}+\frac{4 V^{\prime 2}}{V}}{-f^{\prime} R-2 V^{\prime}}\right]\left[\frac{\left(1-\kappa^{2} f\right)\left(-f^{\prime} R-2 V^{\prime}\right)}{2 V^{2}-f^{\prime 2} R-2 f^{\prime} V^{\prime}}\right] .
$$

In the non-minimal case, the tensor perturbations amplitude of a given mode when leaving the Hubble radius (equation (40)) are given by

$$
A_{T}^{2}=\frac{4 \kappa^{4}}{75 \pi\left(1-\kappa^{2} f\right)}\left[V-\frac{f^{\prime 2} R}{2 V}-\frac{f^{\prime} V^{\prime}}{V}\right]
$$

So, the tensor spectral index (defined by equation (42)) takes the following form

$$
n_{T}=\left(\frac{-f^{\prime} R-2 V^{\prime}}{6 H^{2} V}\right)\left(\frac{\kappa^{2}}{9\left(1-\kappa^{2} f\right) H^{3}}\right)\left(-\frac{f^{\prime 2} R}{2 V}-\frac{f^{\prime} V^{\prime}}{2 V}\right) \times\left(V^{\prime}-\frac{f^{\prime} f^{\prime \prime} R}{V}+\frac{f^{\prime 2} R V^{\prime}}{2 V^{2}}-\frac{f^{\prime \prime} V^{\prime}}{V}+\frac{f^{\prime} V^{\prime \prime}}{V}+\frac{f^{\prime} V^{\prime 2}}{V^{2}}+3 \kappa^{2} H^{2}\right) .
$$

Also, the tensor-to-scalar ratio in the presence of the non- minimal coupling is given by

$$
\begin{aligned}
r_{t-s} \equiv \frac{A_{T}^{2}}{A_{s}^{2}} \simeq \frac{8 \pi}{25 k^{3}} & {\left[\frac{V^{3}-f^{\prime 2} R V-f^{\prime} V^{\prime} V}{V^{\prime 2} \mathcal{C}}\right] } \\
& \times \exp \left[-2 \int\left(\frac{\kappa^{2}\left(f^{\prime} R+2 V V^{\prime}\right)}{6 H^{2} V\left(1-\kappa^{2} f\right)}-\frac{V^{\prime} f^{\prime} R-f^{\prime \prime} R-2 V^{\prime \prime}+\frac{2 V^{\prime 2}}{V}}{-f^{\prime} R-2 V^{\prime}}-\frac{V^{\prime \prime}}{V^{\prime}}+\frac{V^{\prime}}{V}\right) d \phi\right] .
\end{aligned}
$$

Similar to the minimal coupling case, for numerical analysis of the model's parameter space, we firstly solve the integral of equation (92) with the quadratic tachyon potential and $f(\phi)=\xi \phi^{2}$. By solving this integral we obtain

$$
\begin{gathered}
N=\frac{3}{2} \frac{\sigma^{2}\left(\phi_{f}^{2}-\phi_{h c}^{2}\right)}{(12 \xi R+12 \sigma) \xi}-\frac{\xi R}{\xi R+\sigma} \ln \left(\frac{-1+\kappa^{2} \xi \phi_{f}^{2}}{-1+\kappa^{2} \xi \phi_{h c}^{2}}\right) \\
+\frac{\sigma^{2}}{\kappa^{2}(8 \xi R+8 \sigma) \xi^{2}} \ln \left(\frac{-1+\kappa^{2} \xi \phi_{f}^{2}}{-1+\kappa^{2} \xi \phi_{h c}^{2}}\right) \\
-\frac{\sigma}{\xi R+\sigma} \ln \left(\frac{-1+\kappa^{2} \xi \phi_{f}^{2}}{-1+\kappa^{2} \xi \phi_{h c}^{2}}\right)
\end{gathered}
$$

We assume $\phi_{h c} \gg \phi_{f}$ and find $\phi_{h c}$ from equation (122). By substituting the obtained parameter in equa- 

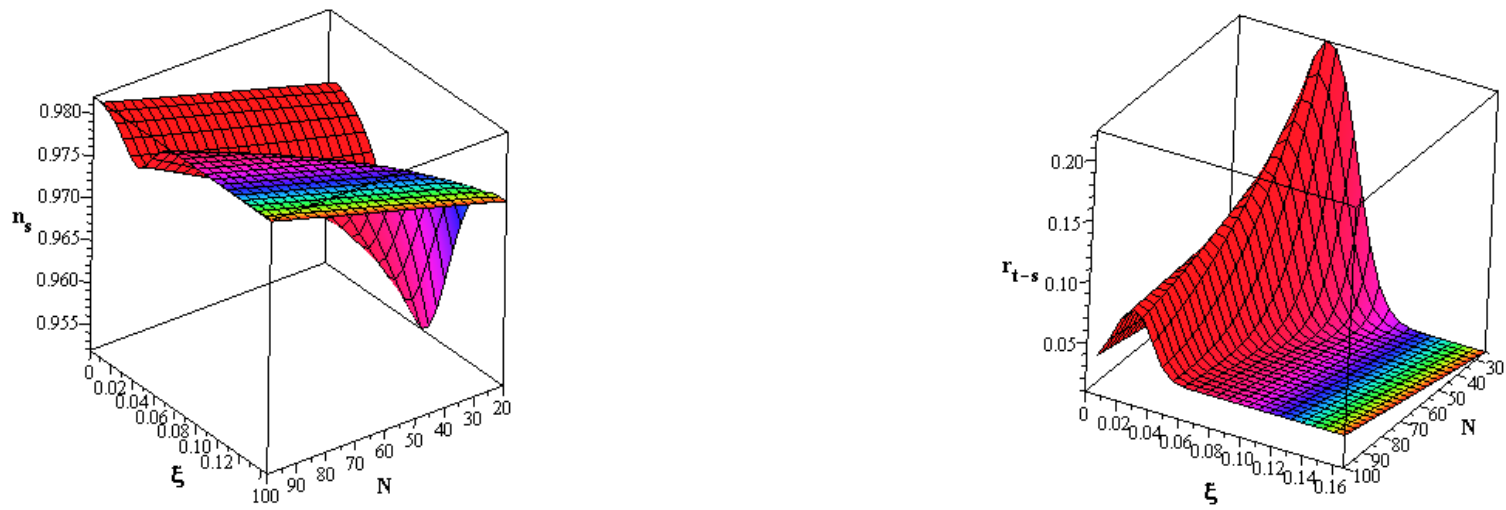

FIG. 13. Evolution of the scalar spectral index (left panel) and the tensor to scalar ratio (right panel) versus the non-minimal coupling and number of e-folds parameter.

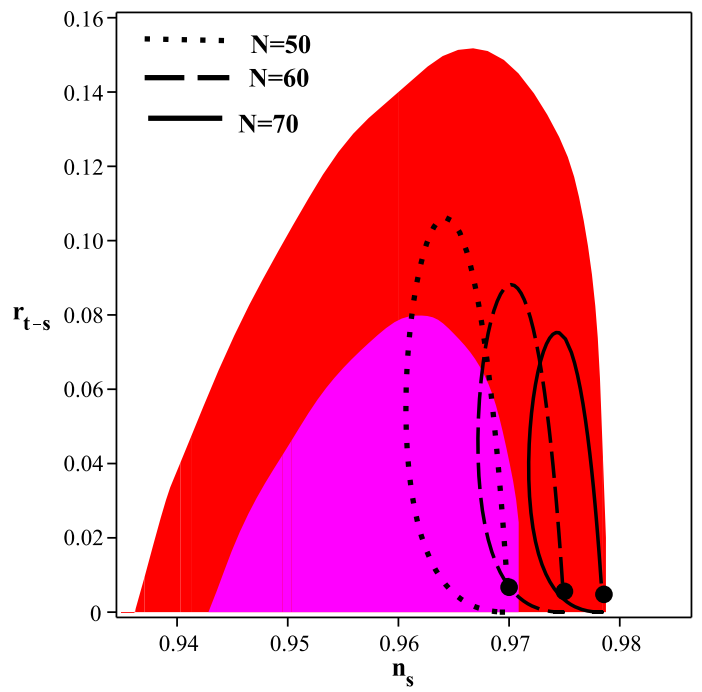

FIG. 14. Behavior of the tensor to scalar ratio with respect to the scalar spectral index for various $\xi$ and for quadratic potential in the background of WMAP9+eCMB+BAO+ $\mathrm{H}_{0}$ data. The two contours are corresponding to the $68 \%$ and $95 \%$ levels of confidence.

tion (118), we plot the evolution of the scalar spectral index with respect to the number of e-folds and the nonminimal coupling parameter. One can see the result in the left panel of figure 13. As figure shows, for all values of $N$, the scalar spectral index firstly decreases by increasing the non-minimal coupling parameter and then increases by more increment of $\xi$. Also, in this case the scalar spectral index is red-tilted. The right panel of figure 13 shows the behavior of the tensor to scalar ratio with respect to the number of e-folds and the nonminimal coupling parameter. This figure confirms that for all values of $N$, tensor to scalar ratio firstly decreases by increasing the non-minimal coupling parameter and then increases by more increment of $\xi$.

The behavior of the tensor to scalar perturbation amplitudes with respect to the scalar spectral index, at the horizon crossing and in the background of WMAP9 $+\mathrm{eCMB}+\mathrm{BAO}+\mathrm{H}_{0}$ data, can be seen in figure 14. This figure has been plotted for $\xi \geq 0$ and for $N=50, N=60$ and $N=70$. This figure also shows that as $\xi$ increases, the scalar spectral index decreases whereas tensor to scalar ratio increases. The reduction of $n_{s}$ and increment of $r_{t-s}$ stop at some values of $\xi$ and after that, the scalar spectral index increases by increment of $\xi$, while $r_{t-s}$ decreases. Note that in the case of quadratic potential, for all values of $\xi$, the values of $r_{t-s}$ and $n_{s}$ are well in the region that is compatible with observation. The dots in the figure show $\xi=0$ and are compatible with corresponding points in figure 2 .

$$
\text { 2. } V=V_{0} e^{-\kappa \sigma \phi}
$$

Now, we consider an exponential potential and solve the integral of equation (92). Solving this integral gives the following expression for number of e-folds parameter

$$
\begin{gathered}
N=\left(-R+2 \frac{\kappa^{2} v \alpha^{2}}{\xi}-\frac{\kappa^{4} v^{2} \alpha^{4}}{\xi^{2} R}+\kappa^{2} \alpha^{2}\right) \mathcal{M} \\
+\left(\sqrt{\xi} R+2 \frac{v R}{\sqrt{\xi}}-\frac{\kappa^{2} v^{2}}{\sqrt{\xi}}-\frac{\kappa^{2} v^{2} \alpha^{2}}{\xi^{\frac{3}{2}}}\right) \mathcal{P}+\left(\frac{1}{2} \kappa^{2} v^{2} R\right. \\
\left.-\xi R^{2}+\frac{1}{2} \frac{\kappa^{2} v^{2} R \alpha^{2}}{\xi}+\frac{1}{2} \kappa^{4} v^{2} \alpha^{2}-\frac{\kappa^{4} v^{3} \alpha^{2}}{\xi}\right) \mathcal{N}
\end{gathered}
$$

where

$$
\mathcal{M}=\frac{\kappa^{2} v^{2} \ln \left(\frac{\xi \phi_{f} R-v \kappa \alpha}{\xi \phi_{h c} R-v \kappa \alpha}\right)}{\xi R^{2}-\kappa^{4} v^{2} \alpha^{2}},
$$



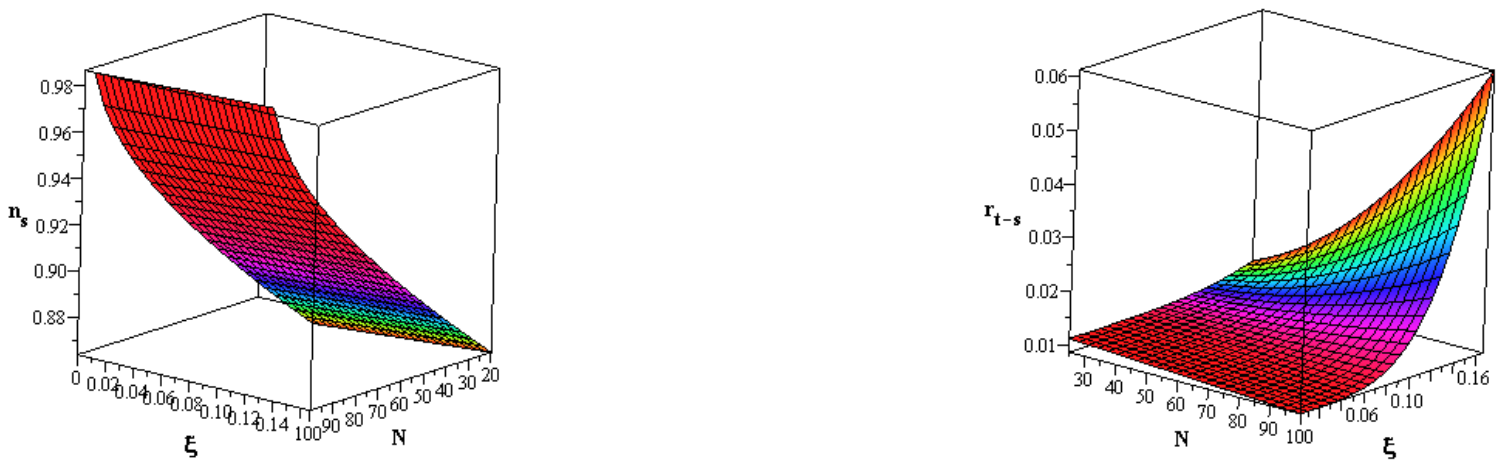

FIG. 15. Evolution of the scalar spectral index (left panel) and the tensor to scalar ratio (right panel) with respect to the non-minimal coupling and number of e-folds parameters.

TABLE IV. The range of the non-minimal coupling parameter which leads to observationally viable scalar spectral index and tensor to scalar ratio for exponential potential and for three different values of $N$.

\begin{tabular}{lccc}
\hline \hline & $N=50$ & $N=60$ & $N=70$ \\
\hline$\xi$ & $0<\xi<0.02$ & $0<\xi<0.033$ & $0<\xi<0.05$ \\
\hline \hline
\end{tabular}

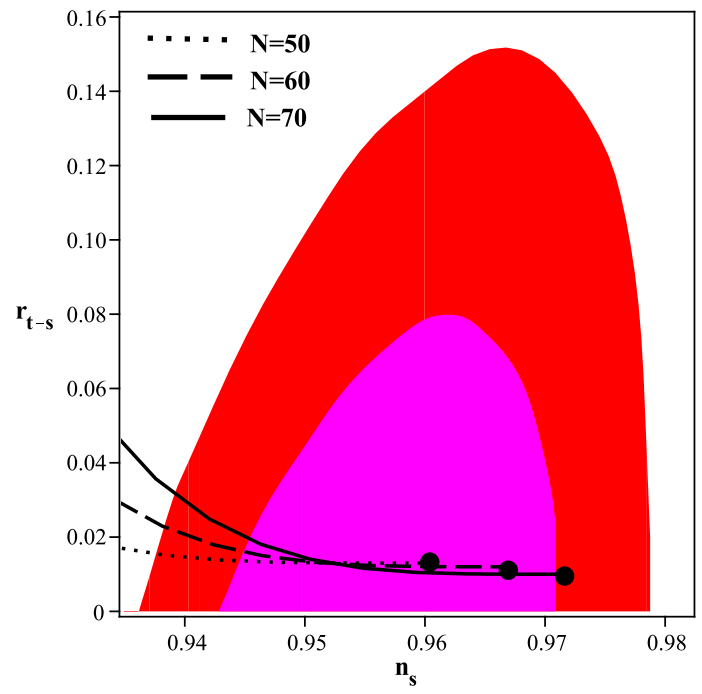

FIG. 16. Behavior of the tensor to scalar ratio with respect to the scalar spectral index for various $\xi$ and for exponential potential in the background of WMAP $9+\mathrm{eCMB}+\mathrm{BAO}+\mathrm{H}_{0}$ data. The two contours are corresponding to the $68 \%$ and $95 \%$ levels of confidence.

$$
\mathcal{N}=\frac{\kappa^{2} v \alpha \ln \left(\frac{-1+\kappa^{2} \xi \phi_{f}^{2}}{-1+\kappa^{2} \xi \phi_{h c}^{2}}\right)}{\xi R^{2}-\kappa^{4} v^{2} \alpha^{2}}
$$

and

$$
\mathcal{P}=\frac{\arctan \left(\kappa \sqrt{\xi} \phi_{f}\right)-\arctan \left(\kappa \sqrt{\xi} \phi_{h c}\right)}{\xi R^{2}-\kappa^{4} v^{2} \alpha^{2}} .
$$

By setting equation (88) equal to unity and finding $\phi_{f}$, we can deduce $\phi_{h c}$ from equation (123). As before, if we substitute expression of $\phi_{h c}$ in equation (118), we can explore the evolution of the scalar spectral index in terms of the number of e-folds and non-minimal coupling parameter (see left panel of figure 15). We see from this figure that for all values of $N$, the scalar spectral index decreases as $\xi$ increases. Also, for all values of $\xi$, the scalar spectral index is red-tilted. The behavior of the tensor to scalar ratio with respect to the number of efolds and the non-minimal coupling parameter is shown in the left panel of figure 15. As figure shows, for all values of the number of e-folds parameter, $r_{t-s}$ increases by increment of $\xi$.

In order to see which values of $\xi$ lead to observationally viable scalar spectral index and tensor to scalar ratio in this model, we show the evolution of $r_{t-s}$ with respect to $n_{s}$ for various $\xi$ in figure 16. We explore this evolution for $N=50, N=60$ and $N=70$. As before, dots in this figure are corresponding to minimal case with $\xi=0$. This figure shows also that depending on the value of $N$, some values of $\xi$ are observationally viable. We have found the range of $\xi$ for mentioned values of $N$ and the results are summarized in table IV.

\section{Intermediate inflation}

In this subsection, we firstly obtain the intermediate potential in the presence of the non-minimal coupling between the tachyon field and Ricci scalar. As the mini- 

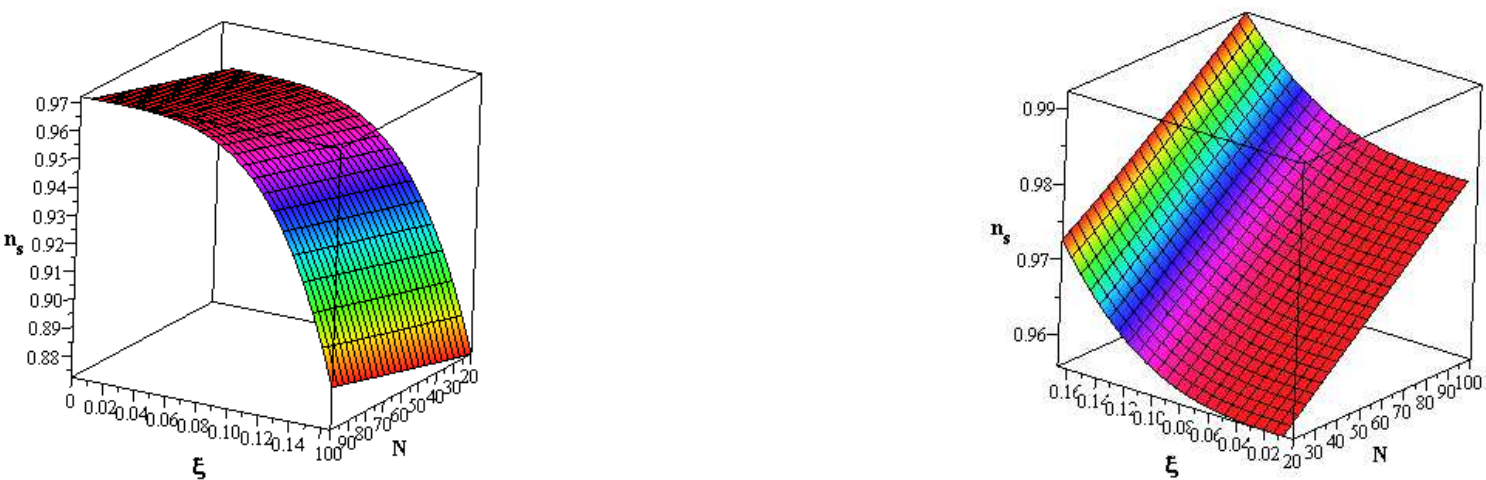

FIG. 17. Evolution of the scalar spectral index with $l=0.4$ (left panel) and $l=0.87$ (right panel) with respect to the non-minimal coupling and number of e-folds parameter.
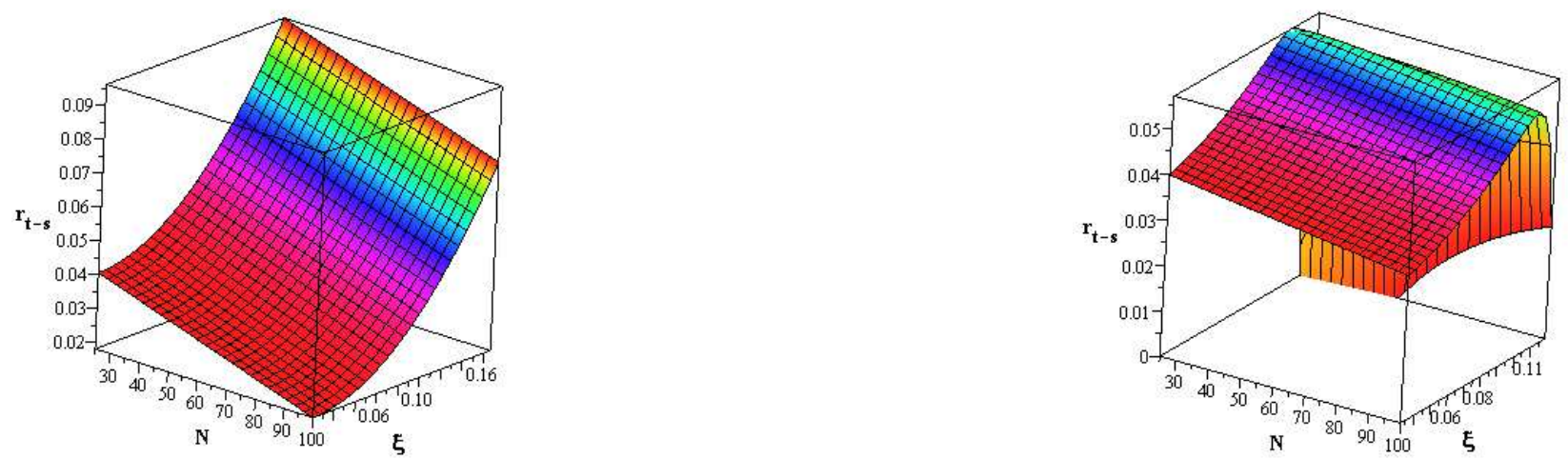

FIG. 18. Evolution of the tensor to scalar ratio with $l=0.4$ (left panel) and $l=0.87$ (right panel) with respect to the non-minimal coupling and the number of e-folds parameter.
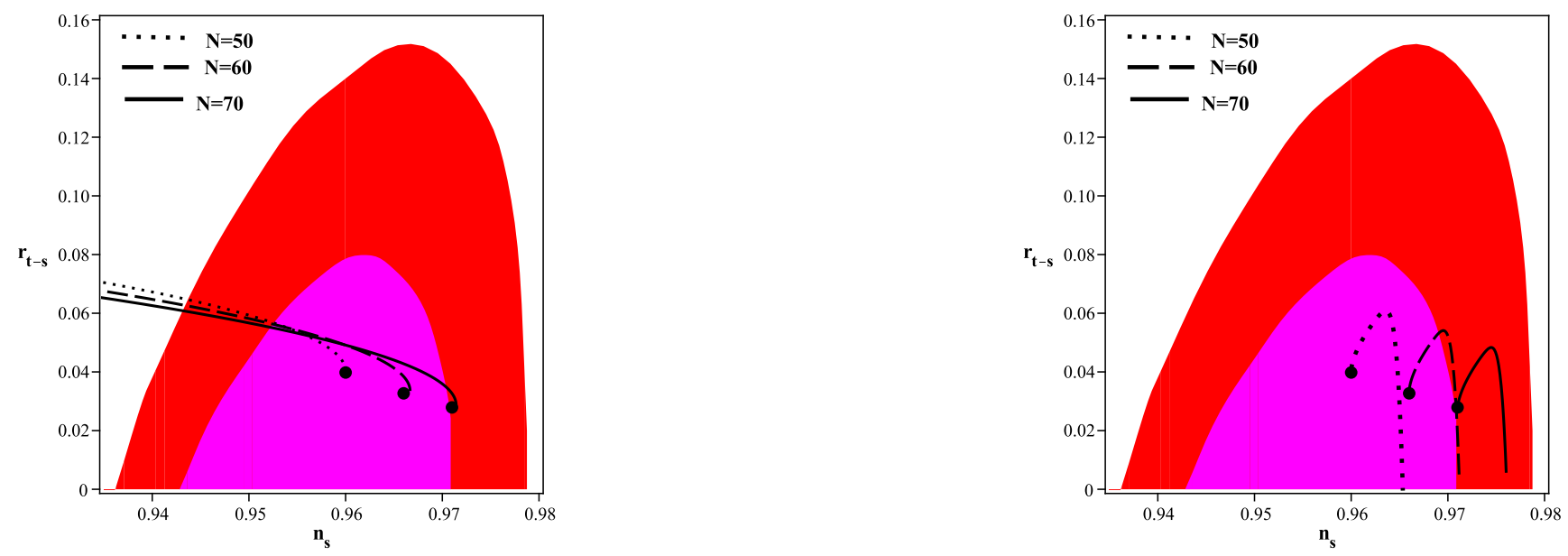

FIG. 19. Behavior of the tensor to scalar ratio with respect to the scalar spectral index for various $\xi$ and for exponential potential in the background of $\mathrm{WMAP} 9+\mathrm{eCMB}+\mathrm{BAO}+\mathrm{H}_{0}$ data. The two contours are corresponding to the $68 \%$ and $95 \%$ levels of confidence. Left panel is corresponding to $l=0.4$ and right panel with $l=0.87$. 
TABLE V. The range of the non-minimal coupling parameter which leads to viable scalar spectral index and tensor to scalar ratio for intermediate potential with $l=0.4$.

\begin{tabular}{lccc}
\hline \hline & $N=50$ & $N=60$ & $N=70$ \\
\hline$\xi$ & $0<\xi<0.149$ & $0<\xi<0.14$ & $0<\xi<0.131$ \\
\hline \hline
\end{tabular}

mal coupling case, we start with scale factor in the form of $a=a_{0} \exp \left(\vartheta t^{l}\right)$ and by using the equations (87) and (88) we obtain

$$
\begin{aligned}
& V=3 l^{2} \vartheta^{2}\left(\frac{b}{\phi^{2}}\right)^{\beta}\left(1-\kappa^{2} \xi \phi^{2}\right) \kappa^{-2}-\frac{\kappa^{2} \xi^{2} \phi^{2+2 \beta}}{1-\kappa^{2} \xi \phi^{2}} \\
& +\xi \phi \vartheta l b^{\beta} \sqrt{\frac{\kappa^{4} \xi^{2} \phi^{2+2 \beta}}{\vartheta^{2} l^{2} b^{\beta}\left(1-\kappa^{2} \xi \phi^{2}\right)^{2}}+6 \frac{l-1}{\vartheta l}}
\end{aligned}
$$

where

$$
b=\frac{3(-2+f)^{2} \vartheta l}{8(1-l)}
$$

and

$$
\beta=\frac{2 l-2}{-2+l}
$$

Now, we solve the integral of equation (92) to obtain the number of e-folds parameter. The result is so complicated and we present it in Appendix A. As other cases, by finding $\phi_{h c}$ from $N$ and substituting it in (118), we can explore the evolution of the scalar spectral index versus the number of e-folds and the non-minimal coupling parameter (figure 17). The left panel of figure 17 has been plotted with $l=0.4$ and the right panel with $l=0.87$. This figure shows that both with $l=0.4$ and $l=0.87$, for all values of $N$, the scalar spectral index decreases as the non-minimal coupling parameter increases. The evolution of the tensor to scalar ratio versus the number of e-folds and the non-minimal coupling parameter is shown in figure 18. With $l=0.4$, for all values of $N$, the tensor to scalar ratio increases as $\xi$ increases. With $l=0.87$, for all values of $N$, this ratio first increases by increment of $\xi$ and then decreases by more increment of $\xi$. Note that for all $l \leq 0.63$ the behavior of the scalar spectral index and tensor to scalar ratio are more or less similar to the left panel of figures 17 and 18 and for all $l>0.63$ the behavior of $n_{s}$ and $r_{t-s}$ are similar to the right panel of figures 17 and 18 respectively.

Figure 19 shows the evolution of the tensor to scalar ratio with respect to the scalar spectral index in the background of $\mathrm{WMAP} 9+\mathrm{eCMB}+\mathrm{BAO}+\mathrm{H}_{0}$ data for $N=50$, $N=60$ and $N=70$. As figure shows, with $l=0.87$ (the right panel), for all values of $\xi$, the tensor to scalar ratio and scalar spectral index are compatible with observational data. With $l=0.4$, only in some range of the non-minimal coupling parameter, $n_{s}$ and $r_{t-s}$ lie in the region that observational data shows. One can see the corresponding range of $\xi$ for three values of $N$ in table $\mathrm{V}$

\section{Non-Gaussianity}

To study the non-gaussianity in this model we should keep the gradient term in the equation of motion of the scalar field. So, in the presence of the non-minimal coupling we have

$$
3 H \dot{\phi}=a^{-2} \nabla^{2} \phi-\frac{V^{\prime}}{V}+\frac{f^{\prime} R}{2 V}+\Xi .
$$

By expanding equation (130) up to the second order in the fluctuations of the tachyon field $\left(\delta \phi=\delta \phi^{(1)}+\delta \phi^{(2)}\right)$ and in a time interval $t_{n}-t_{n-1}=\frac{1}{H}$, we obtain the following expression for the first and second order fluctuations

$$
\begin{aligned}
\frac{d}{d t}\left(\delta \phi^{(1)}(\mathbf{k}, t)\right)=\frac{1}{3 H}\{ & {\left[-k^{2}-\frac{2 V^{\prime \prime}+f^{\prime \prime} R}{2 V}+\frac{V^{\prime 2}}{V^{2}}\right.} \\
& \left.\left.-\frac{f^{\prime} R V^{\prime}}{2 V^{2}}\right] \delta \phi^{(1)}+\Xi\right\},
\end{aligned}
$$

and

$$
\begin{aligned}
& \frac{d}{d t}\left(\delta \phi^{(2)}(\mathbf{k}, t)\right)=\frac{1}{3 H}\left\{\left[-k^{2}-\frac{2 V^{\prime \prime}+f^{\prime \prime} R}{2 V}+\frac{V^{\prime 2}}{V^{2}}\right.\right. \\
& \left.-\frac{f^{\prime} R V^{\prime}}{2 V^{2}}\right] \delta \phi^{(2)}+\left[\frac{3 V^{\prime} V^{\prime \prime}}{V^{2}}-\frac{V^{\prime \prime \prime}}{V}-\frac{2 V^{\prime 3}}{V^{3}}-\frac{f^{\prime \prime \prime} R}{2 V}\right. \\
& \left.\left.+\frac{f^{\prime \prime} V^{\prime} R}{V^{2}}+\frac{f^{\prime} R V^{\prime \prime}}{2 V^{2}}-\frac{f^{\prime} R V^{\prime 2}}{V^{3}}\right]\left(\delta \phi^{(1)}\right)^{2}\right\} .
\end{aligned}
$$

The solution of the equations (131) and (132) are given by

$$
\begin{array}{r}
\delta \phi^{(1)}(\mathbf{k}, t)=\Theta\left(k, t-t_{n-1}\right) \int_{t_{n-1}}^{t} d t^{\prime} \frac{\Xi}{3 H} \Theta^{-1}\left(k, t-t_{n-1}\right) \\
+\Theta\left(k, t-t_{n-1}\right) \delta \phi^{(1)}\left(\mathbf{k} e^{-H\left(t_{n}-t_{n-1}\right)}, t_{n-1}\right), \quad(133)
\end{array}
$$

and 


$$
\begin{array}{r}
\delta \phi^{(2)}(\mathbf{k}, t)=\Theta\left(k, t-t_{n-1}\right) \int_{t_{n-1}}^{t} d t^{\prime} \chi\left(k, t^{\prime}\right)\left[\int \frac{d p^{3}}{(2 \pi)^{3}} \delta \phi^{(1)}\left(\mathbf{p}, t^{\prime}\right) \delta \phi^{(1)}\left(\mathbf{k}-\mathbf{p}, t^{\prime}\right)\right] \Theta^{-1}\left(k, t^{\prime}-t_{n-1}\right) \\
+\Theta\left(k, t-t_{n-1}\right) \delta \phi^{(2)}\left(\mathbf{k} e^{-H\left(t_{n}-t_{n-1}\right)}, t_{n-1}\right)
\end{array}
$$

where

$$
\begin{array}{r}
\Theta=\exp \left[-\int_{t_{0}}^{t}\left(\frac{k^{2}}{3 H}+\frac{2 V^{\prime \prime}+f^{\prime \prime} R}{6 H V}-\frac{V^{\prime 2}}{3 H V^{2}}\right.\right. \\
\left.\left.-\frac{f^{\prime} R V^{\prime}}{6 H V^{2}}\right) d t^{\prime}\right],
\end{array}
$$

and

$$
\begin{array}{r}
\chi=\frac{2 V^{\prime} V^{\prime \prime}}{H V^{2}}-\frac{2 V^{\prime \prime \prime}}{3 H V}-\frac{4 V^{\prime 3}}{3 H V^{3}}-\frac{f^{\prime \prime \prime} R}{3 H V}+\frac{2 f^{\prime \prime} V^{\prime} R}{3 H V^{2}} \\
+\frac{f^{\prime} R V^{\prime \prime}}{3 H V^{2}}-\frac{2 f^{\prime} R V^{\prime 2}}{3 H V^{3}} .
\end{array}
$$

In the presence of the non-minimal coupling between the tachyon field and the Ricci scalar, the freezeout momentum $k_{F}$ is expressed by the following condition

$$
\frac{k_{F}^{2}}{3 H^{2}}+\frac{2 V^{\prime \prime}+f^{\prime \prime} R}{6 H^{2} V}-\frac{V^{\prime 2}}{3 H^{2} V^{2}}-\frac{f^{\prime} R V^{\prime}}{6 H^{2} V^{2}}=1
$$

So, we find

$$
k_{F}=\sqrt{\frac{6 H^{2} V^{2}-\left(2 V^{\prime \prime}+f^{\prime \prime} R\right) V+2 V^{\prime 2}+f^{\prime} R V^{\prime}}{2 V^{2}}} .
$$

Now, we compute the three-point correlation function of the tachyon fluctuations at large scale, at the time about 50 e-folds before the end of inflation, by using equations (133) and (134). The result is as follows

$$
\begin{gathered}
\left\langle\delta \phi\left(\mathbf{k}_{1}, t\right) \delta \phi\left(\mathbf{k}_{2}, t\right) \delta \phi\left(\mathbf{k}_{3}, t\right)\right\rangle=\Theta\left(k_{3}, t-t_{50}-\frac{1}{H}\right) \times \\
\int_{t_{50}-\frac{1}{H}}^{t_{50}} d t^{\prime} \Theta^{-1}\left(k_{3}, t^{\prime}-t_{50}-\frac{1}{H}\right) \chi\left(k_{3}, t^{\prime}\right) \\
{\left[\int \frac{d^{3} p}{(2 \pi)^{3}}\left\langle\delta \phi^{(1)}\left(\mathbf{k}_{1}, t_{1}\right) \delta \phi^{(1)}\left(\mathbf{p}, t^{\prime}\right)\right\rangle\right.} \\
\left.\left\langle\delta \phi^{(1)}\left(\mathbf{k}_{2}, t_{2}\right) \delta \phi^{(1)}\left(\mathbf{k}_{3}-\mathbf{p}, t^{\prime}\right)\right\rangle\right] \\
+\Theta\left(k_{3}, t-t_{50}-\frac{1}{H}\right)\left\langle\delta \phi^{(1)}\left(\mathbf{k}_{1}, t_{50}\right) \delta \phi^{(1)}\left(\mathbf{k}_{2}, t_{50}\right)\right. \\
\left.\delta \phi^{(1)}\left(\mathbf{k}_{3} e^{-1}, t_{50}-\frac{1}{H}\right)\right\rangle+\left(\mathbf{k}_{1} \leftrightarrow \mathbf{k}_{3}\right)+\left(\mathbf{k}_{2} \leftrightarrow \mathbf{k}_{3}\right)
\end{gathered}
$$

On the scales with $k<k_{F}$, we can take $\Theta$ about unity and $\chi$ as a constant. So we have

$$
\begin{gathered}
\left\langle\delta \phi\left(\mathbf{k}_{1}, t\right) \delta \phi\left(\mathbf{k}_{2}, t\right) \delta \phi\left(\mathbf{k}_{3}, t\right)\right\rangle \approx \chi\left(k_{F}, t_{F}\right) \frac{1}{H} \ln \left(\frac{k_{F}}{H}\right) \times \\
{\left[\int \frac{d^{3} p}{(2 \pi)^{3}}\left\langle\delta \phi^{(1)}\left(\mathbf{k}_{1}, t_{1}\right) \delta \phi^{(1)}\left(\mathbf{p}, t^{\prime}\right)\right\rangle\right.} \\
\left\langle\delta \phi^{(1)}\left(\mathbf{k}_{2}, t_{2}\right) \delta \phi^{(1)}\left(\mathbf{k}_{3}-\mathbf{p}, t^{\prime}\right)\right\rangle \\
\left.+\left(\mathbf{k}_{1} \leftrightarrow \mathbf{k}_{3}\right)+\left(\mathbf{k}_{2} \leftrightarrow \mathbf{k}_{3}\right)\right]
\end{gathered}
$$

The bispectrum for the single field inflation models in the slow-roll limit is given by the following expression

$$
\begin{gathered}
\left\langle\Phi\left(\mathbf{k}_{1}\right) \Phi\left(\mathbf{k}_{2}\right) \Phi\left(\mathbf{k}_{3}\right)\right\rangle=2 f_{N L}(2 \pi)^{3} \delta^{3}\left(\mathbf{k}_{1}+\mathbf{k}_{2}+\mathbf{k}_{3}\right) \\
{\left[P_{\Phi}\left(\mathbf{k}_{1}\right)+P_{\Phi}\left(\mathbf{k}_{2}\right)+\text { permutations }\right] .}
\end{gathered}
$$

On the other hand, the gravitational field potential, in the presence of the non-minimal coupling is given by

$$
\Phi=-\frac{1}{2} \frac{H}{\dot{\phi}} \frac{\left[\Sigma+\frac{\kappa^{2} f^{\prime} \Upsilon}{1-\kappa^{2} f}\right]^{-1}}{\left(1-\frac{f^{\prime} R}{6 H V}\right)} \delta \phi
$$

where

$$
\Sigma=\frac{1}{3}-\frac{2 f^{\prime} f^{\prime \prime} R V-f^{\prime 2} R V^{\prime}}{6 V^{\prime} V^{2}}-\frac{f^{\prime \prime} V+f^{\prime} \frac{V^{\prime \prime} V}{V^{\prime}}-f^{\prime} V^{\prime}}{3 V^{2}},
$$

and

$$
\Upsilon=\frac{V}{3 V^{\prime}}-\frac{f^{\prime 2} R}{6 V V^{\prime}}-\frac{f^{\prime}}{3 V}
$$

So, from equations (142), (140) and (141) we find

$$
\begin{aligned}
& f_{N L}=2 \frac{\dot{\phi}}{H}\left(1-\frac{f^{\prime} R}{6 H V}\right)\left(\Sigma+\frac{\kappa^{2} f^{\prime} \Upsilon}{1-\kappa^{2} f}\right) \\
& \times\left[\frac { 1 } { H } \operatorname { l n } ( \frac { k _ { F } } { H } ) \left(\frac{2 V^{\prime} V^{\prime \prime}}{H V^{2}}-\frac{2 V^{\prime \prime \prime}}{3 H V}-\frac{4 V^{\prime 3}}{3 H V^{3}}\right.\right. \\
& \left.\left.-\frac{f^{\prime \prime \prime} R}{3 H V}+\frac{2 f^{\prime \prime} V^{\prime} R}{3 H V^{2}}+\frac{f^{\prime} R V^{\prime \prime}}{3 H V^{2}}-\frac{2 f^{\prime} R V^{\prime 2}}{3 H V^{3}}\right)\right]
\end{aligned}
$$

In figure 20 we have plotted the behavior of $f_{N L}$ versus $\dot{\phi}$ and $\xi$ for $N=50$ and for three types of potentials: quadratic, exponential and intermediate potential. As the figure shows, for quadratic potential, similar to the minimal case, $f_{N L}$ is positive. In this case, the value of non-gaussianity parameter decreases as the non-minimal coupling increases. With an exponential potential, the 

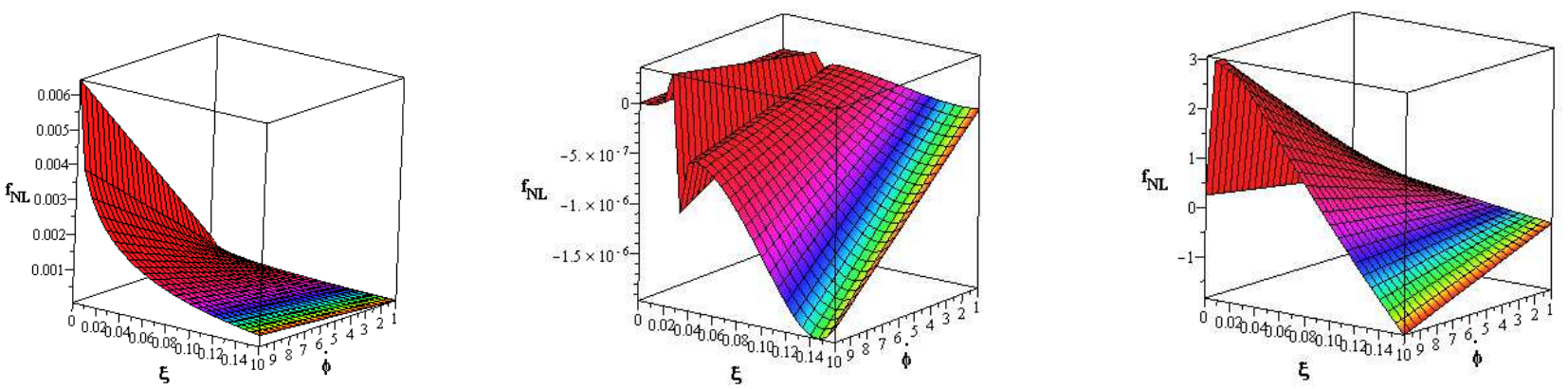

FIG. 20. Non-gaussianity versus $\xi$ and $\dot{\phi}$ for $N=50$ for quadratic (left panel), exponential (middle panel) and intermediate potential (right panel) in the non-minimal coupling setup.

non-gaussianity parameter can be either positive or negative, depending on the value of $\xi$. In this case, $f_{N L}$ decreases as the non-minimal coupling parameter increases. But, at some value of $\xi$ it reaches a minimum and then increases by more increment of the non-minimal coupling. With an intermediate potential, depending on the value of the non-minimal coupling, the non-gaussianity parameter is either positive or negative (note that in the minimal coupling case, $f_{N L}$ for an intermediate potential is always positive). With this potential, $f_{N L}$ increases as the non-minimal coupling parameter increases until it reaches a maximum and then decreases as $\xi$ increases. For intermediate potential we have set $l=0.4$.

\section{Cosmological dynamics and late time acceleration}

In this section, similar to the minimal coupling case, we study cosmological dynamics of a non-minimally coupled tachyon field by using the dynamical system technique. In the presence of a non-minimally coupled tachyon field and ordinary component(s), the Friedmann equation of our setup takes the following form

$$
\left(1-\kappa^{2} f\right) H^{2}=\frac{\kappa^{2}}{3}\left[\frac{V(\phi)}{\sqrt{1-\dot{\phi}^{2}}}+3 f^{\prime} H \dot{\phi}+\rho_{m}\right] .
$$

Now, as usual we should define some dimensionless variables in order to translate our cosmological equations in the language of the autonomous dynamical system. In this non-minimal coupling framework, we use the dimensionless parameters defined in (66) and in addition to two new parameters defined as follows

$$
z=\kappa \sqrt{\xi} \phi, \quad u=\frac{\kappa \sqrt{\xi}}{H}
$$

With these dimensionless parameters, the Friedmann equation (146) can be written as follows

$$
1=\frac{y^{2}}{\sqrt{1-x^{2}}}+2 u z x+z^{2}+d^{2},
$$

which gives a constraint on the parameters of the model. As usual this constraint can be used to express one of the dimensionless parameters in terms of the others.

In the presence of a non-minimally coupled tachyon field and in terms of the new variables, the acceleration equation takes the following form

$$
\begin{gathered}
\frac{\dot{H}}{H^{2}}=\left[-\frac{3}{2} \frac{y^{2} x^{2}}{\sqrt{1-x^{2}}}-u z x+\frac{3}{2} y^{2} \sqrt{1-x^{2}}\right. \\
-\left(1-x^{2}\right)\left(3 u z x+\sqrt{3} u z y \alpha+4 u^{2} z^{2} y^{2}\right)+u^{2} x^{2} \\
\left.-\frac{3}{2} d^{2}(1+\omega)\right] \times\left[1-z^{2}+2 \frac{u^{2} z^{2}}{2}\left(1-x^{2}\right)^{\frac{3}{2}}\right]^{-1},
\end{gathered}
$$

and the scalar field's equation of motion becomes

$$
\frac{\ddot{\phi}}{H}=\left(1-x^{2}\right)(-3 x-\sqrt{3} \alpha y)-\frac{2 u z}{y^{2}}\left(1-x^{2}\right)^{\frac{3}{2}}\left(\frac{\dot{H}}{H^{2}}+2\right) \text {. }
$$

Also, the effective equation of state parameter in terms of the dimensionless parameters is given by the following expression

$$
\omega_{e f f}=\frac{\frac{\sqrt{3} y^{2} \alpha x}{\sqrt{1-x^{2}}}-\frac{y^{2} x}{\left(1-x^{2}\right)^{\frac{3}{2}}} \frac{\ddot{\phi}}{H}-2 u^{2} x^{2}-2 u z \frac{\dot{H}}{H^{2}}-2 u z \frac{\ddot{\phi}}{H}-2 u z x-2 z^{2} \frac{\dot{H}}{H^{2}}}{\frac{3 y^{2}}{\sqrt{1-x^{2}}}+6 u z x+3 z^{2}}-1 .
$$


In the next step, we obtain the autonomous equations with a non-minimally coupled tachyon field

$$
\begin{gathered}
\frac{d x}{d \tau}=\left(1-x^{2}\right)(-3 x-\sqrt{3} \alpha y)-\frac{2 u z\left(1-x^{2}\right)^{\frac{3}{2}}}{y^{2}}\left(\frac{\dot{H}}{H^{2}}+2\right) \\
\frac{d y}{d \tau}=\frac{\sqrt{3}}{2} y^{2} \alpha x-y \frac{\dot{H}}{H^{2}}, \\
\frac{d z}{d \tau}=u z
\end{gathered}
$$

and

$$
\frac{d u}{d \tau}=-u \frac{\dot{H}}{H^{2}}
$$

where $\frac{\dot{H}}{H^{2}}$ is given by equation (149). By finding the roots of the system of equations (152)-(155), we find 5 critical lines denoted as $\mathcal{L}_{1}, \mathcal{L}_{2}, \mathcal{L}_{3}, \mathcal{L}_{4}$ and $\mathcal{L}_{5}$. The characters of these critical lines are shown in table [V] The eigenvalues $\lambda_{2 \mathcal{L}_{1}}, \lambda_{3 \mathcal{L}_{1}}$ and $\lambda_{4 \mathcal{L}_{1}}$ in table 4 , are defined in Appendix $\mathrm{B}$ and $\lambda_{\mathcal{L}}$ is given by the following expression

$$
\lambda_{\mathcal{L}}=\frac{3}{2} \omega+\frac{3}{2}-\frac{3}{2} z^{2}-\frac{3}{2} z^{2} \omega
$$

Also, we have

$$
\begin{array}{r}
x_{\mathcal{L}_{1}}=\frac{2 \sqrt{3}}{9}\left(-6-6 z^{4}+12 z^{2}-3 z^{2} \sqrt{4 z^{4}-8 z^{2}+13}\right. \\
\left.+3 \sqrt{4 z^{4}-8 z^{2}+13}\right)^{\frac{1}{2}}, \quad \text { (157) }
\end{array}
$$

and

$$
\begin{aligned}
y_{\mathcal{L}_{1}}=\frac{1}{3}\left(-6-6 z^{4}\right. & +12 z^{2}-3 z^{2} \sqrt{4 z^{4}-8 z^{2}+13} \\
& \left.+3 \sqrt{4 z^{4}-8 z^{2}+13}\right)^{\frac{1}{2}} \cdot \quad
\end{aligned}
$$

The critical line $\mathcal{L}_{2}$, which is effectively ordinary component dominated, is a saddle line. This means that if the universe during its evolution reaches this state, evolves to other states. Critical lines $\mathcal{L}_{3}$ and $\mathcal{L}_{4}$ are unstable (repeller) lines, because their eigenvalues are positive. These lines are effectively ordinary component dominated solutions and can be relevant to the early time. The critical lines $\mathcal{L}_{1}$ and $\mathcal{L}_{5}$, which are effectively dark energy dominated, are stable critical lines, meaning that if the universe reaches this state, remains there forever. This can be corresponding to the late time in the history of the universe expansion. Figure 21 shows the phase space trajectories of the parameters space in 3 dimensions for $\omega=0$.

As we have seen, for the minimal coupling case the stable point was not able to explain the late time accelerating phase of the universe expansion. However, in

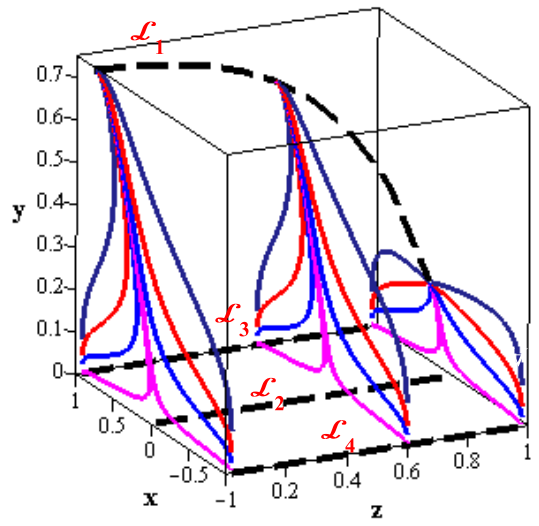

FIG. 21. The phase space trajectories of a model with ordinary component(s) and a non-minimally coupled tachyon field. We assumed that, the ordinary component is dust with $\omega=0$. There is one stable critical line, $\mathcal{L}_{1}$, and 3 unstable critical lines $\left(\mathcal{L}_{2}, \mathcal{L}_{3}\right.$ and $\left.\mathcal{L}_{4}\right)$. The trajectories are plotted for just three values of $z$ : $z(0)=0.02, z(0)=0.6$ and $z(0)=0.98$. We have not plotted the critical line $\mathcal{L}_{5}$ to avoid complexity in the figure.

the non-minimal coupling case the stable lines $\mathcal{L}_{1}$ and $\mathcal{L}_{5}$ have the capability of explaining the accelerating phase of the universe expansion. This is because for all values of $z$, the effective equation of state parameter, say for $\mathcal{L}_{1}$, which is defined as

$$
\omega_{\mathcal{L}_{1}}=\frac{1}{9} \frac{-4 A^{\frac{3}{2}} z^{2}+4 A^{\frac{3}{2}}+z^{2} B^{2} A-9 z^{2} A+9 A}{(z-1)(z+1)\left(A+3 z^{2} B\right)},
$$

where

$$
\begin{gathered}
A=-6-6 z^{4}+12 z^{2}-3 z^{2} \sqrt{4 z^{4}-8 z^{2}+13} \\
+3 \sqrt{4 z^{4}-8 z^{2}+13},
\end{gathered}
$$

and

$$
\begin{gathered}
B=\left(17+8 z^{4}-16 z^{2}+4 z^{2} \sqrt{4 z^{4}-8 z^{2}+13}\right. \\
\left.-4 \sqrt{4 z^{4}-8 z^{2}+13}\right)^{\frac{1}{2}}
\end{gathered}
$$

is always below $-\frac{1}{3}$. So, the universe in this case experiences the late time accelerating phase of expansion. It is interesting to note that the effective equation of state parameter of the model shows effective phantom behavior in some subspaces of the model parameter space.

Figure 22 shows the evolution of the effective equation of state parameter in the presence of ordinary component(s) and a non-minimally coupled tachyon field. The effective equation of state parameter tends to a stable 
TABLE VI. Location, Eigenvalues and Dynamical Characters of the Critical Lines.

\begin{tabular}{ccccc}
\hline \hline Line & $(x, y, z, u)$ & eigenvalues & $\omega_{\text {eff }}$ & stability \\
\hline $\mathcal{L}_{1}$ & $\left(x_{\mathcal{L}_{1}}, y_{\mathcal{L}_{1}}, z, 0\right)$ & $\left(0, \lambda_{2 \mathcal{L}_{1}}, \lambda_{3 \mathcal{L}_{1}}, \lambda_{4} \mathcal{L}_{1}\right)$ & $\omega_{\mathcal{L}_{1}}$ & stable \\
\hline $\mathcal{L}_{2}$ & $(0,0, z, 0)$ & $\left(0, \lambda_{\mathcal{L}}, \lambda_{\mathcal{L}}, \lambda_{\mathcal{L}}\right)$ & $\omega$ & unstable \\
\hline $\mathcal{L}_{3}$ & $(1,0, z, 0)$ & $\left(0,6, \lambda_{\mathcal{L}}, \lambda_{\mathcal{L}}\right.$ & $\omega$ & unstable \\
\hline $\mathcal{L}_{4}$ & $(-1,0, z, 0)$ & $\left(0,6, \lambda_{\mathcal{L}}, \lambda_{\mathcal{L}}\right)$ & $\omega$ & unstable \\
\hline $\mathcal{L}_{5}$ & $\left(-x_{\mathcal{L}_{1}},-y_{\mathcal{L}_{1}}, z, 0\right)$ & $\left(0, \lambda_{2 \mathcal{L}_{1}}, \lambda_{3 \mathcal{L}_{1}}, \lambda_{4 \mathcal{L}_{1}}\right)$ & $\omega$ & stable \\
\hline \hline
\end{tabular}
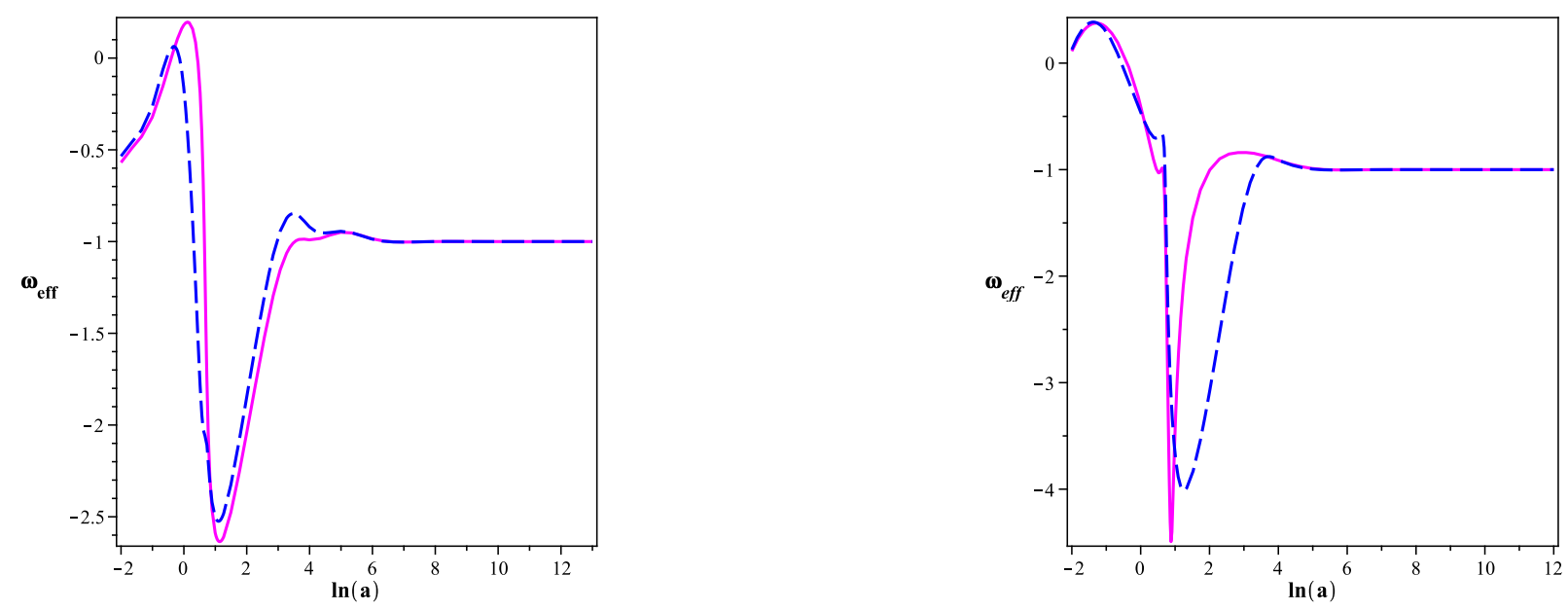

FIG. 22. The behavior of the effective equation of state parameter in the non-minimal coupling case. The left panel is corresponding to $\omega=0$ (dust) and the right one is corresponding to $\omega=\frac{1}{3}$ (radiation). As the figure shows, $\omega_{e f f}$ evolves and tends to the stable state with $\omega_{\text {eff }}=-1$. The figure is plotted with $x(0)=0.99, y(0)=0.01, z(0)=0.2$ and $u(0)=0.1$ for magenta curve and $x(0)=0.75, y(0)=0.05, z(0)=0.2$ and $u(0)=0.1$ for blue curve.

state which is corresponding to the $\Lambda \mathrm{CDM}$ scenario. So, the universe with a non-minimally coupled tachyon field experiences the accelerating phase of expansion in its history.

Now, we analyze the statefinder parameters diagnostic. As for the minimal coupling case, we rewrite equation (75) in terms of the newly introduced dimensionless parameters as follows

$$
r=\frac{\mathcal{D}}{\mathcal{B}}-\frac{\mathcal{A K}}{\mathcal{B}^{2}}+2 \frac{\mathcal{A}^{2}}{\mathcal{B}^{2}}+3 \frac{\mathcal{A}}{\mathcal{B}}+1
$$

where $\mathcal{A}, \mathcal{B}, \mathcal{D}$ and $\mathcal{K}$ are defined as

$$
\begin{aligned}
\mathcal{A}=- & \frac{3}{2} \frac{y^{2} x^{2}}{\sqrt{1-x^{2}}}-u z x+\frac{3}{2} y^{2} \sqrt{1-x^{2}}-\left(1-x^{2}\right)(3 u z x \\
& \left.+\sqrt{3} u z y \alpha+4 u^{2} z^{2} y^{2}\right)+u^{2} x^{2}-\frac{3}{2} d^{2}(1+\omega), \quad(163
\end{aligned}
$$

$$
\mathcal{B}=1-z^{2}+2 \frac{u^{2} z^{2}}{2}\left(1-x^{2}\right)^{\frac{3}{2}}
$$

$$
\begin{gathered}
\mathcal{D}=-\frac{3 y \frac{d y}{d N}}{\sqrt{1-x^{2}}}-\frac{3}{2} \frac{y^{2} x \frac{d x}{d N}}{\left(1-x^{2}\right)^{\frac{3}{2}}}-z x \frac{d u}{d N}-u x \frac{d z}{d N} \\
-u z \frac{d x}{d N}+3 y y^{\prime} \sqrt{1-x^{2}}-\frac{3}{2} \frac{y^{2} x \frac{d x}{d N}}{\sqrt{1-x^{2}}}+2 u x^{2} \frac{d u}{d N} \\
+2 x \frac{d x}{d N}\left(3 u z x+\sqrt{3} u z y \alpha+4 u^{2} z^{2} y^{2}\right)-\left(1-x^{2}\right) \\
\left(3 z x \frac{d u}{d N}+3 u x \frac{d z}{d N}+3 u z \frac{d x}{d N}+\sqrt{3} z y \alpha \frac{d u}{d N}\right. \\
+\sqrt{3} u y \alpha \frac{d z}{d N}+\sqrt{3} u z \alpha \frac{d y}{d N}+8 u \frac{d u}{d N} z^{2} y^{2}+8 z \frac{d z}{d N} u^{2} y^{2} \\
\left.+y \frac{d y}{d N} u^{2} z^{2}\right)+2 u^{2} x \frac{d x}{d N}-3 d \frac{d d}{d N}(1+\omega), \quad(165) \\
\mathcal{K}=+\left(1-x^{2}\right)^{\frac{3}{2}}\left(\frac{4 u \frac{d u}{d N} z^{2}+4 u^{2} z \frac{d z}{d N}}{y^{2}}-\frac{4 u^{2} z^{2} \frac{d y}{d N}}{y^{3}}\right) \\
-2 z \frac{d z}{d N}-\frac{6 u^{2} z^{2} x \frac{d x}{d N}}{y^{2}}\left(1-x^{2}\right)(166)
\end{gathered}
$$

The parameter $s$, can be obtained by substituting equation (162) into equation (74).

For numerical analysis of the statefinder diagnostic, we study the phase plane of the pairs $\{r, q\},\{s, q\}$ and 

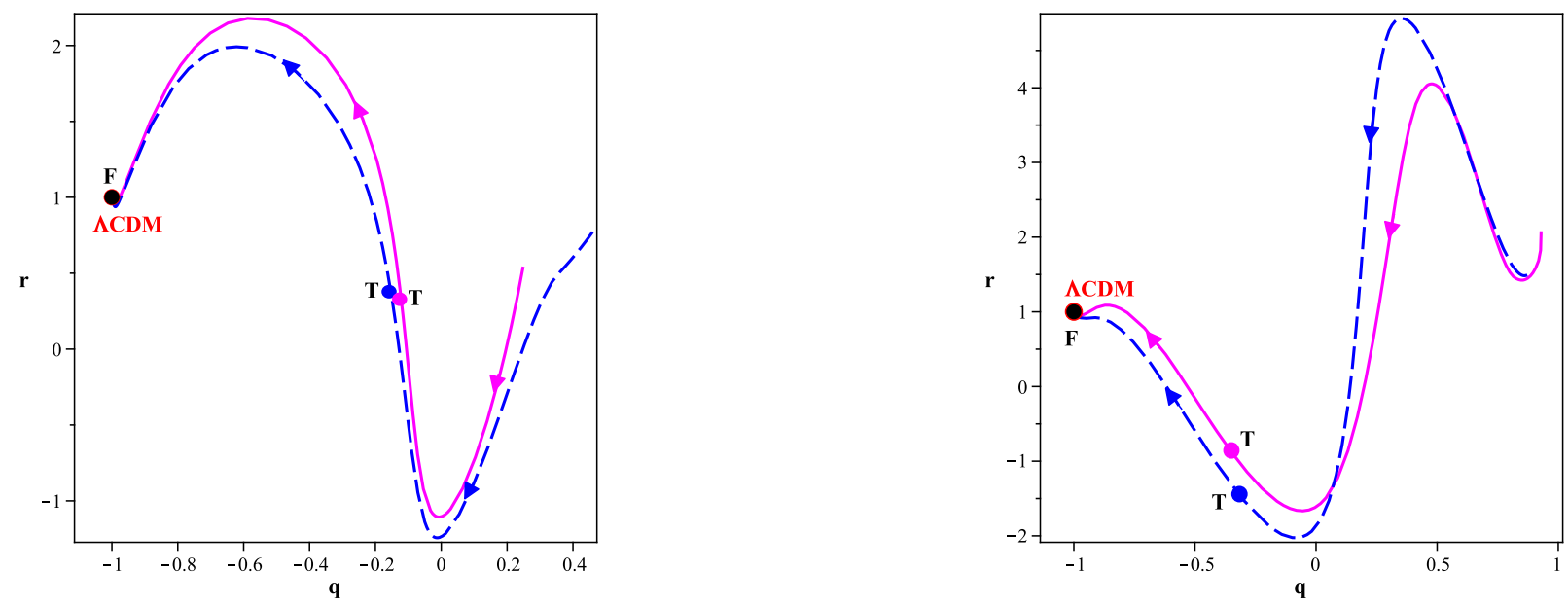

FIG. 23. The trajectories in $\{r, q\}$ phase plan with $\omega=0$ (left panel) and $\omega=\frac{1}{3}$ (right panel). The initial conditions are $x(0)=0.99, y(0)=0.01, z(0)=0.2$ and $u(0)=0.1$ for magenta trajectory and $x(0)=0.75, y(0)=0.05, z(0)=0.2$ and $u(0)=0.1$ for blue trajectory. The magenta and blue highlighted dots (specified by $T$ ) are current values of $\{r, q\}$ in the model with non-minimally coupled tachyon field. The black dot (specified by $F$ ) is the stable state of $\{r, q\}$ in future. Also the red $\operatorname{dot}($ marked by $\Lambda \mathrm{CDM})$ is the value of statefinder $\{r, q\}$ in a $\Lambda$ CDM scenario.
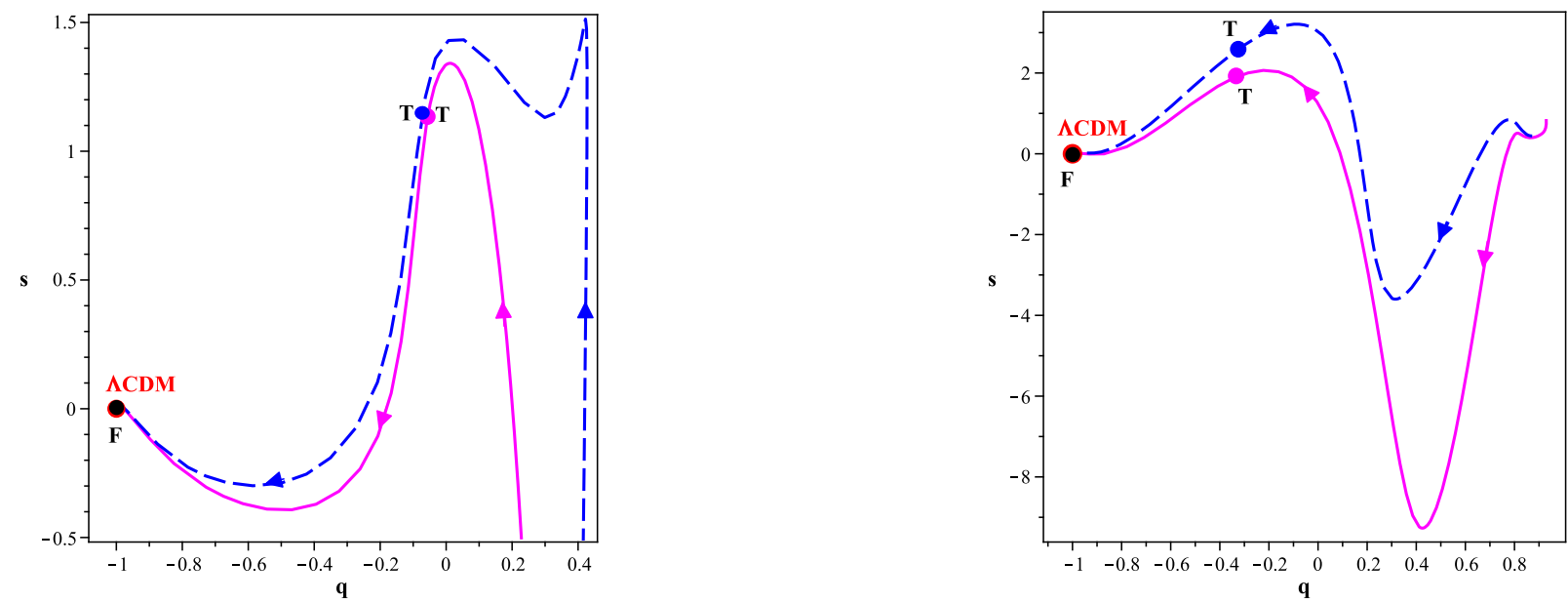

FIG. 24. The trajectories in $\{s, q\}$ phase plan with $\omega=0$ (left panel) and $\omega=\frac{1}{3}$ (right panel). The initial conditions are $x(0)=0.99, y(0)=0.01, z(0)=0.2$ and $u(0)=0.1$ for magenta trajectory and $x(0)=0.75, y(0)=0.05, z(0)=0.2$ and $u(0)=0.1$ for blue trajectory. The magenta and blue dots (specified by $T$ ) are current values of $\{s, q\}$ in the model with non-minimally coupled tachyon field. The black dot $(F)$ is the stable state of $\{s, q\}$ in the future. Also the red dot (remarked by $\Lambda \mathrm{CDM})$ is the value of statefinder $\{s, q\}$ in a $\Lambda \mathrm{CDM}$ scenario.

$\{r, s\}$. The left panel of figure 23]shows the trajectories of the phase plane $\{r, q\}$ for $\omega=0$. Also, the right panel of this figure shows the trajectories in the phase plan $\{r, q\}$ for $\omega=\frac{1}{3}$. As this figure shows, in a model with ordinary components (dust or radiation) and a non-minimally coupled tachyon field, the trajectories of $\{r, q\}$ plan terminate in a stable point in future, which is corresponding to the $\Lambda$ CDM model (with $\{r, q\}=\{0,-1\}$ ). As before, magenta and blue highlighted dots show the current values of the parameters. The trajectories of phase plane $\{s, q\}$ is shown in figure 24 for $\omega=0$ (left panel) and $\omega=\frac{1}{3}$ (right panel). In this phase plane also, the trajectories evolve to a stable point in future which is corre- sponding to the $\Lambda$ CDM scenario (with $\{s, q\}=\{1,-1\}$ ). Another important pair is $\{r, s\}$, which its phase plane has been plotted in figure 25. As other cases, the left panel is corresponding to $\omega=0$ and the right panel is corresponding to $\omega=\frac{1}{3}$. The trajectories in phase plan $\{r, s\}$ reach a point corresponding to $\Lambda \mathrm{CDM}$ model.

\section{SUMMARY}

We have studied cosmological dynamics of a tachyon field as a field responsible for initial inflation and late time cosmic acceleration. Firstly, we have considered a 

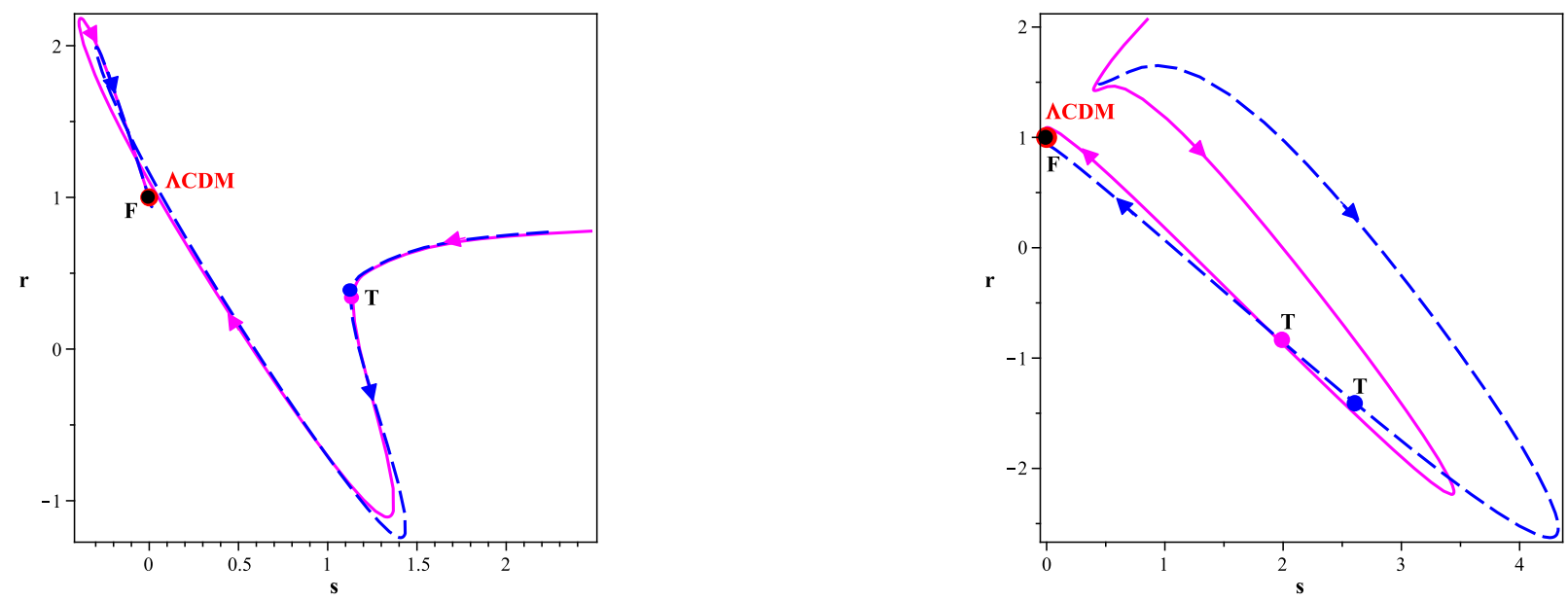

FIG. 25. The trajectories in $\{r, s\}$ phase plan with $\omega=0$ (left panel) and $\omega=\frac{1}{3}$ (right panel). The initial conditions are $x(0)=0.99, y(0)=0.01, z(0)=0.2$ and $u(0)=0.1$ for magenta trajectory and $x(0)=0.75, y(0)=0.05, z(0)=0.2$ and $u(0)=0.1$ for blue trajectory. The magenta and blue highlighted dots (specified by $T$ ) are current values of $\{r, s\}$ in the model with non-minimally coupled tachyon field. The black dot $(F)$ is the stable state of $\{r, s\}$ in the future. Also red dot (marked by $\Lambda \mathrm{CDM})$ is the value of statefinder $\{r, s\}$ in a $\Lambda$ CDM scenario.

tachyon field which is minimally coupled to gravity and we have explored its dynamic both in the inflationary stage and late time in the history of the universe. In the inflationary stage, we have calculated the inflation parameters and perturbations with details. If there is only one field in inflation period as responsible for inflation, the adiabatic perturbations are generated. On the other hand, if there is more than one scalar field in a model, or a scalar field interacts with other fields such as the Ricci scalar, the isocurvature perturbations can be generated. So, in the minimal coupling case, the perturbation are adiabatic (meaning that $\delta p_{\text {nad }}=0$ and so $\dot{\zeta}=0$ ). By adopting three types of potential, a quadratic tachyon potential, an exponential potential and an intermediate potential, we have performed a numerical analysis of the model's parameter space and the results have been shown in figures and tables. Also, we have compared the minimally coupled tachyon model with observational data by plotting the evolution of the tensor to scalar ratio with respect to the scalar spectral index in the background of the WMAP9 $+\mathrm{eCMB}+\mathrm{BAO}+\mathrm{H}_{0}$ data. By this comparison we have found the range of intermediate parameter, $l$, for which $n_{s}$ and $r_{t-s}$ are compatible with recent observational data. We have also studied the non-gaussianity of perturbations for a minimally coupled tachyon field model. As we have shown, in the minimal coupling case, both with quadratic and intermediate potentials $f_{N L}$ is positive whereas with the exponential potential $f_{N L}$ is either positive or negative, depending on the value of $N$.

Within a dynamical system approach, the cosmological dynamics and especially late time cosmic evolution of a model with ordinary component(s) and the minimally coupled tachyon field has been explored. By finding the roots of the autonomous equations, we have obtained the critical points of the phase space. We have found three unstable critical points (which are ordinary component(s) dominated solutions) and one stable fixed points (which is tachyon field dominated) in the minimal setup. The effective equation of state parameter corresponding to the stable point is larger then $-\frac{1}{3}$. This means that, the minimally coupled tachyon field model cannot explain the late time acceleration of the universe. Also, the statefinder diagnostic of the minimal case has been studied. By plotting the phase plan of the pairs $\{r, q\}$, $\{s, q\}$ and $\{r, s\}$ we have seen that the trajectories of the minimal case tend to the state which has distance from $\Lambda \mathrm{CDM}$ model.

In the next step, we have considered a tachyon field which is non-minimally coupled to the Ricci scalar. we have obtained the inflation parameters and perturbations with details. We have seen that due to the presence of the non-minimal coupling between the tachyon field and Ricci scalar, the perturbations are non-adiabatic and there are isocurvature perturbations in the setup. Actually, in the presence of the non-minimal coupling there is non-vanishing, non-adiabatic pressure leading to the non-vanishing time evolution of the primordial curvature perturbations. By adopting $f(\phi)=\xi \phi^{2}$, we have numerically analyzed the models parameter space in the inflationary stage for three types of potential. The results have been shown in figures. Note that we have obtained the intermediate potential in the presence of the non-minimal coupling of the tachyon field and Ricci scalar, which is more complicated than the corresponding parameter in the minimal coupling case. In the nonminimal case, we have plotted the behavior of the tensor to scalar ratio versus the scalar spectral index in the background of the WMAP9+eCMB $+\mathrm{BAO}+\mathrm{H}_{0}$ data, for $\xi \geq 0$. With a quadratic potential and with $N=50$, $N=60$ and $N=70$, for all values of $\xi$, the tensor 
to scalar ratio and scalar spectral index are compatible with observational data. With an exponential potential, $n_{s}$ and $r_{t-s}$ are compatible with observational data in some range of the non-minimal coupling parameter. In the case of intermediate potential, there are two states. If $l>0.63$, scalar spectral index and tensor to scalar ratio, for all values of $\xi$, lie in the region compatible with observational data. If $l \leq 0.63$, the scalar spectral index and tensor to scalar ratio lie in the viable domain only in some range of the non-minimal coupling parameter. We have studied the non-gaussianity of perturbations in this model in the presence of the non-minimal coupling. In the non-minimal coupling case, both with exponential and intermediate potentials $f_{N L}$ is either positive or negative, depending on the value of $\xi$. But, with a quadratic potential the non-gaussianity parameter is positive and well in the range supported by recent observations. Similar to the minimal case, the cosmological dynamics of the setup has been considered with details in a dynam- ical system technique. We have found that, there are three unstable critical lines in the model with ordinary component(s) and the non-minimally coupled tachyon field. These unstable lines are effectively ordinary component(s) dominated solutions. In the non-minimal coupling case, there are two stable critical lines which are effectively dark energy dominated. In contrast to the minimal coupling case, a model with a non-minimally coupled tachyon field has capability to explain the late time accelerating phase of the universe expansion. By studying the statefinder pairs in phase plans, we have found that the non-minimal model reaches a stable state which is corresponding to the $\Lambda \mathrm{CDM}$ scenario. This behavior is independent of the initial conditions.

\section{Appendix A: Number of e-folds parameter for an intermediate potential in the presence of the non-minimal coupling}

$$
\begin{aligned}
& N=\sqrt{\frac{l-1}{\vartheta l}}\left[\left(\frac{\sqrt{6} \kappa^{2} b^{\beta}\left(1+\frac{1}{4 \beta}\right)}{6 \vartheta l\left(-\kappa^{2} \xi\right)^{\frac{1}{2}+\beta}}+\frac{\sqrt{6} \kappa^{2}\left(1+\frac{1}{\beta}+b^{\beta}\right)}{144 \vartheta^{3} l^{3} \beta\left(-\kappa^{2} \xi\right)^{\frac{1}{2}+\beta} b^{\beta}} \frac{1}{\left(\frac{b^{-\beta}}{3 \vartheta l(l-1)}+1\right)}\right)\left(\frac{1}{2} \grave{\mathcal{B}} \grave{\mathcal{O}}-\frac{\grave{\mathcal{B}}}{1+2 \beta}\right)\right. \\
& \left.+\frac{\sqrt{6} \kappa^{4}\left(1+\frac{1}{16 b^{2 \beta}}\right)}{36 \vartheta^{2} l^{2}(l-1)\left(-\kappa^{2} \xi\right)^{\frac{1}{2}+\beta}}\left(\frac{\grave{\vartheta}\left(5 \kappa^{2} \xi \phi^{2}+4 \beta \kappa^{2} \xi \phi^{2}-4 \beta-3\right)}{\left(-1+\kappa^{2} \xi \phi^{2}\right)^{2}}+\frac{\left(16 \beta+3+16 \beta^{2}\right) \grave{\vartheta}}{2 \vartheta^{2} l^{2} \beta} \dot{\mathcal{O}}\right)\right]- \\
& \frac{\kappa^{2}}{6 b^{2} l^{2}\left(-\kappa^{2} \xi\right)^{2 \beta} b^{\beta}}\left(\frac{\grave{\mathcal{C}}\left(\kappa^{2} \xi \phi^{2}-1-2 \beta\right)}{\beta\left(-1+\kappa^{2} \xi \phi^{2}\right)}-\grave{\mathcal{C}}(2+4 \beta) \grave{\mathcal{O}}\right)-\frac{\kappa^{2} \phi^{4 \beta}\left(1-2 b^{\beta}\right)}{24 \vartheta^{2} l^{2} \beta b^{\beta}}\left(\left(6 \beta+4 \beta^{2}+2\right) \grave{\mathcal{O}}\right. \\
& \left.-\frac{1-2 \kappa^{2} \xi \phi^{2}+3 \beta+\kappa^{4} \xi^{2} \phi^{4}-2 \beta^{2} \kappa^{2} \xi \phi^{2}+2 \beta^{2}-4 \beta \kappa^{2} \xi \phi^{2}}{\beta\left(-1+\kappa^{2} \xi \phi^{2}\right)^{2}}\right)-\frac{\kappa^{4} \xi \phi^{8 \beta}\left(\frac{8 \beta+8}{\beta}\right)}{54 b^{\beta} \vartheta^{4} l^{4} \beta^{-1}}\left(\left(6 \beta+1+8 \beta^{2}\right) \grave{\mathcal{O}}\right. \\
& \left.+\frac{\left(1-3 \kappa^{2} \xi \phi^{2}+6 \beta+8 \beta \phi^{4} \kappa^{4} \xi^{2}+8 \beta^{2} \kappa^{4} \xi^{2} \phi^{4}-16 \kappa^{2} \xi \phi^{2} \beta^{2}+3 \kappa^{4} \xi^{2} \phi^{4}+8 \beta^{2}-14 \beta \kappa^{2} \xi \phi^{2}\right)}{\beta\left(-1+\kappa^{2} \xi \phi^{2}\right)^{3}}\right) \\
& +\frac{\kappa^{4} \xi \phi^{8 \beta} \vartheta^{-4} l^{-4} \beta^{-1}}{54(4+4 \beta)(\beta+1) b^{\beta}}\left(\frac{12 \kappa^{2} \xi \phi^{2}-3+32 \beta^{3} \kappa^{6} \xi^{3} \phi^{6}-96 \beta^{3} \kappa^{4} \xi^{2} \phi^{4}+96 \beta^{3} \kappa^{2} \xi \phi^{2}-18 \kappa^{4} \xi^{2} \phi^{4}}{\left(-1+\kappa^{2} \xi \phi^{2}\right)^{4}}\right. \\
& +\frac{76 \beta \kappa^{2} \xi \phi^{2}-22 \beta-48 \beta^{2}-32 \beta^{3}-90 \beta \phi^{4} \kappa^{4} \xi^{2}-160 \beta^{2} \kappa^{4} \xi^{2} \phi^{4}+152 \kappa^{2} \xi \phi^{2} \beta^{2}+56 \beta^{2} \kappa^{6} \xi^{3} \phi^{6}}{\left(-1+\kappa^{2} \xi \phi^{2}\right)^{4}} \\
& \left.+\frac{36 \beta \phi^{6} \kappa^{6} \xi^{3}+12 \kappa^{6} \xi^{3} \phi^{6}}{\left(-1+\kappa^{2} \xi \phi^{2}\right)^{4}}+4 \beta(\beta+1)\left(3+22 \beta+48 \beta^{2}+32 \beta^{3}\right) \grave{\mathcal{O}}\right)+\left[\frac{\sqrt{6} \xi \phi^{1+8 \beta}(7+8 \beta)}{2592 \vartheta^{4} l^{4} \beta(l-1) b^{2 \beta}}\right. \\
& \left(\frac{73 \kappa^{4} \xi^{2} \phi^{4}-55 \kappa^{2} \xi \phi^{2}-56 \beta-448 \beta^{2}-512 \beta^{3}+232 \beta \kappa^{2} \xi \phi^{2}+1472 \kappa^{2} \xi \phi^{2} \beta^{2}-360 \beta \phi^{4} \kappa^{4} \xi^{2}}{16(7 / 2+4 \beta)\left(-1+\kappa^{2} \xi \phi^{2}\right)^{4} \kappa^{6} \xi^{3}}\right. \\
& +\frac{1536 \beta^{3} \kappa^{2} \xi \phi^{2}+15 \kappa^{6} \xi^{3} \phi^{6}-1600 \beta^{2} \kappa^{4} \xi^{2} \phi^{4}-1536 \beta^{3} \kappa^{4} \xi^{2} \phi^{4}+576 \beta^{2} \kappa^{6} \xi^{3} \phi^{6}+512 \beta^{3} \kappa^{6} \xi^{3} \phi^{6}}{(7 / 2+4 \beta)\left(-1+\kappa^{2} \xi \phi^{2}\right)^{4} \kappa^{6} \xi^{3}} \\
& \left.+\frac{15+184 \beta \phi^{6} \kappa^{6} \xi^{3}}{(7 / 2+4 \beta)\left(-1+\kappa^{2} \xi \phi^{2}\right)^{4} \kappa^{6} \xi^{3}}+\frac{1}{32} \frac{\left(15+184 \beta+576 \beta^{2}+512 \beta^{3}\right)(-1+8 \beta) \grave{\mathcal{O}}}{(7 / 2+4 \beta) \kappa^{6} \xi^{3}}\right)+\left(\frac{3}{2} \grave{\mathcal{D}}\right. \\
& \left.\left.\left(1+8 \beta+12 \beta^{2}\right) \grave{\mathcal{O}}-\frac{\left(5 \kappa^{2} \xi \phi^{2}+6 \beta \kappa^{2} \xi \phi^{2}-6 \beta-3\right) \grave{\mathcal{D}}}{\left(-1+\kappa^{2} \xi \phi^{2}\right)^{2}}\right) \frac{\sqrt{6} \kappa^{4} \xi}{72 \vartheta^{3} l^{3} \beta b^{\beta}\left(-\kappa^{2} \xi\right)^{\frac{1}{2}+3 \beta}}\right] \sqrt{\frac{\vartheta l}{l-1}}+\prod+\bigwedge
\end{aligned}
$$


where

$$
\begin{aligned}
& \Pi=-\left[\frac{\sqrt{6} \kappa^{4} \xi}{216 b^{5} l^{5} \beta\left(-\kappa^{2} \xi\right)^{\frac{1}{2}+3 \beta} b^{\beta}}\left(\frac{\left(5 \kappa^{2} \xi \phi^{2}+6 \beta \kappa^{2} \xi \phi^{2}-6 \beta-3\right) \grave{\mathcal{D}}}{\left(-1+\kappa^{2} \xi \phi^{2}\right)^{2}}+\frac{3}{2} \grave{\mathcal{D}}\left(1+8 \beta+12 \beta^{2}\right) \grave{\mathcal{O}}\right)+\left(\frac{3}{2}\right.\right. \\
& \left(8 \beta+1+12 \beta^{2}\right)(6 \beta-1) \grave{\mathcal{O}}+\frac{8 \kappa^{2} \xi \phi^{2}+3 \kappa^{4} \xi^{2} \phi^{4}+36 \beta^{2}-36 \beta \kappa^{2} \xi \phi^{2}-72 \beta^{2} \kappa^{2} \xi \phi^{2}+24 \kappa^{4} \xi^{2} \phi^{4} \beta}{\left(-1+\kappa^{2} \xi \phi^{2}\right)^{3}} \\
& \left.\left.+\frac{36 \beta^{2} \kappa^{4} \xi^{2} \phi^{4}-3+12 \beta}{\left(-1+\kappa^{2} \xi \phi^{2}\right)^{3}}\right) \frac{\sqrt{6} \kappa^{4} \xi^{2} \vartheta^{-2} l^{-2} \beta^{-1} \dot{\mathcal{E}}}{648\left(-\kappa^{2} \xi\right)^{\frac{1}{2}+3 \beta}}\right] \frac{\sqrt{\frac{l-1}{\vartheta l}}}{\left(\frac{b^{-\beta}}{3 \vartheta l(l-1)}+1\right)}+\frac{\sqrt{\frac{l-1}{\vartheta l}}}{\vartheta^{4} l^{4}(l-1)}\left[\left(-\frac{1}{2}(15+92 \beta\right.\right. \\
& \left.+64 \beta^{3}+144 \beta^{2}\right) \grave{\mathcal{O}}-\frac{-40 \kappa^{2} \xi \phi^{2}+33 \kappa^{4} \xi^{2} \phi^{4}+16 \beta^{2}+15-72 \beta \kappa^{2} \xi \phi^{2}-32 \beta^{2} \kappa^{2} \xi \phi^{2}+40 \kappa^{4} \xi^{2} \phi^{4} \beta}{\left(-1+\kappa^{2} \xi \phi^{2}\right)^{3}} \\
& \left.+\frac{16 \beta^{2} \kappa^{4} \xi^{2} \phi^{4}+32 \beta}{\left(-1+\kappa^{2} \xi \phi^{2}\right)^{3}}\right) \frac{\sqrt{6} \kappa^{4} \xi \beta^{-1} \grave{\mathcal{F}}}{108\left(-\kappa^{2} \xi\right)^{\frac{1}{2}+2 \beta}}+\frac{\sqrt{6} \kappa^{6} \xi^{2}\left(\frac{\grave{\mathcal{G}}}{\beta}+\grave{\mathcal{H}}\right)}{1296 b^{\beta}\left(-\kappa^{2} \xi\right)^{\frac{1}{2}+4 \beta}}\left(\frac{1}{2} \mathcal{G}(8 \beta-1) \grave{\mathcal{O}}(15+184 \beta+\right. \\
& \left.576 \beta^{2}+512 \beta^{3}\right)+\frac{73 \kappa^{4} \xi^{2} \phi^{4}-448 \beta^{2}-512 \beta^{3}+15+232 \beta \kappa^{2} \xi \phi^{2}+1472 \beta^{2} \kappa^{2} \xi \phi^{2}-360 \kappa^{4} \xi^{2} \phi^{4} \beta}{\left(-1+\kappa^{2} \xi \phi^{2}\right)^{4}} \\
& \frac{184 \kappa^{6} \xi^{3} \phi^{6} \beta-1600 \beta^{2} \kappa^{4} \xi^{2} \phi^{4}-1536 \beta^{3} \kappa^{4} \xi^{2} \phi^{4}+1536 \beta^{3} \kappa^{2} \xi \phi^{2}+576 \beta^{2} \kappa^{6} \xi^{3} \phi^{6}+512 \beta^{3} \kappa^{6} \xi^{3} \phi^{6}}{\left(-1+\kappa^{2} \xi \phi^{2}\right)} \\
& \left(-1+\kappa^{2} \xi \phi^{2}\right)^{4} \\
& \left.+\frac{15 \kappa^{6} \xi^{3} \phi^{6}-56 \beta}{\left(-1+\kappa^{2} \xi \phi^{2}\right)^{4}}\right)-\frac{\sqrt{6} \kappa^{6} \xi^{2} \beta^{-1} \grave{\mathcal{K}}}{6480 b^{\beta}\left(-\kappa^{2} \xi\right)^{\frac{1}{2}+4 \beta}}\left(\frac{\grave{\mathcal{O}}}{2}\left(1408 \beta+8192 \beta^{3}+4096 \beta^{4}+105+5504 \beta^{2}\right)(1-8 \beta)\right. \\
& -\frac{790 \kappa^{6} \xi^{3} \phi^{6}-896 \kappa^{4} \xi^{2} \phi^{4}+3584 \beta^{2}+7168 \beta^{3} 4096 \beta^{4}+105 \kappa^{8} \xi^{4} \phi^{8}+272 \beta+24576 \beta^{4} \kappa^{4} \xi^{2} \phi^{4}}{\left(-1+\kappa^{2} \xi \phi^{2}\right)^{5}}+ \\
& \frac{1408 \kappa^{8} \xi^{4} \phi^{8} \beta+490 \kappa^{2} \xi \phi^{2}-16384 \beta^{4} \kappa^{2} \xi \phi^{2}-1456 \beta \kappa^{2} \xi \phi^{2}-16384 \beta^{4} \kappa^{6} \xi^{3} \phi^{6}+5504 \beta^{2} \kappa^{8} \xi^{4} \phi^{8}}{\left(-1+\kappa^{2} \xi \phi^{2}\right)^{5}} \\
& \left(-1+\kappa^{2} \xi \phi^{2}\right)^{5} \\
& +\frac{8192 \beta^{3} \kappa^{8} \xi^{4} \phi^{8}-105+4096 \beta^{4} \kappa^{8} \xi^{4} \phi^{8}-15744 \beta^{2} \kappa^{2} \xi \phi^{2}+3120 \kappa^{4} \xi^{2} \phi^{4} \beta+26240 \beta^{2} \kappa^{4} \xi^{2} \phi^{4}}{\left(-1+\kappa^{2} \xi \phi^{2}\right)^{5}} \\
& \left.+\frac{46080 \beta^{3} \kappa^{4} \xi^{2} \phi^{4}-29696 \beta^{3} \kappa^{2} \xi \phi^{2}-3344 \kappa^{6} \xi^{3} \phi^{6} \beta-19584 \beta^{2} \kappa^{6} \xi^{3} \phi^{6}-31744 \beta^{3} \kappa^{6} \xi^{3} \phi^{6}}{\left(-1+\kappa^{2} \xi \phi^{2}\right)^{5}}\right)+(4 \\
& (1-4 \beta) \beta\left(48 \beta^{2}+32 \beta^{3}+3+22 \beta\right) \grave{\mathcal{O}}-\frac{15 \kappa^{2} \xi \phi^{2}+38 \beta \kappa^{2} \xi \phi^{2}-30 \kappa^{4} \xi^{2} \phi^{4}+40 \beta^{2}+160 \beta^{3}+128 \beta^{4}}{\left(-1+\kappa^{2} \xi \phi^{2}\right)^{5}} \\
& \underline{352 \beta^{2} \kappa^{4} \xi^{2} \phi^{4}-3-46 \kappa^{4} \xi^{2} \phi^{4} \beta-192 \beta^{2} \kappa^{2} \xi \phi^{2}-288 \beta^{2} \kappa^{6} \xi^{3} \phi^{6}-736 \beta^{3} \kappa^{6} \xi^{3} \phi^{6}+1056 \beta^{3} \kappa^{4} \xi^{2} \phi^{4}} \\
& \left(-1+\kappa^{2} \xi \phi^{2}\right)^{5} \\
& +\frac{88 \beta^{2} \kappa^{8} \xi^{4} \phi^{8}-672 \beta^{3} \kappa^{2} \xi \phi^{2}-10 \beta+6 \kappa^{6} \xi^{3} \phi^{6} \beta+192 \beta^{3} \kappa^{8} \xi^{4} \phi^{8}+12 \kappa^{8} \xi^{4} \phi^{8} \beta-512 \beta^{4} \kappa^{2} \xi \phi^{2}}{\left(-1+\kappa^{2} \xi \phi^{2}\right)^{5}}+ \\
& \left.\left.\frac{128 \beta^{4} \kappa^{8} \xi^{4} \phi^{8}+30 \kappa^{6} \xi^{3} \phi^{6}-512 \beta^{4} \kappa^{6} \xi^{3} \phi^{6}+768 \beta^{4} \kappa^{4} \xi^{2} \phi^{4}}{\left(-1+\kappa^{2} \xi \phi^{2}\right)^{5}}\right) \frac{\kappa^{6} \xi^{2} \beta^{-1} \vartheta^{-1} l^{-1} \grave{\mathcal{I}}}{6480 b^{2 \beta}\left(-\kappa^{2} \xi\right)^{4 \beta}}\right]
\end{aligned}
$$




$$
\begin{aligned}
& \bigwedge=-\frac{\kappa^{4} \xi}{2\left(-\kappa^{2} \xi\right)^{3 \beta}}\left(\frac{2-6 \kappa^{2} \xi \phi^{2}+9 \beta+6 \kappa^{4} \xi^{2} \phi^{4}+9 \beta^{2}-21 \beta \kappa^{2} \xi \phi^{2}+12 \kappa^{4} \xi^{2} \phi^{4} \beta+9 \beta^{2} \kappa^{4} \xi^{2} \phi^{4}-18 \beta^{2} \kappa^{2} \xi \phi^{2}}{\left(-1+\kappa^{2} \xi \phi^{2}\right)^{3}}+3 \beta\right. \\
& \left.\left(9 \beta+9 \beta^{2}+2\right) \grave{\mathcal{O}}\right) \frac{\left(\frac{\grave{\mathcal{O}}}{108}+\frac{\grave{\mathcal{O}}}{108} \beta+\frac{\grave{\mathcal{J}}\left(1+3 \vartheta l^{2} b^{\beta}-3 \vartheta l b^{\beta}\right)}{27 b^{\beta}}\right)}{\beta l^{4} \vartheta^{4}\left(1+3 \vartheta l^{2} b^{\beta}-3 \vartheta l b^{\beta}\right)}+\frac{\vartheta^{-1} l^{-1} \beta^{-1} \grave{\mathcal{R}}}{\left(1+3 \vartheta l^{2} b^{\beta}-3 \vartheta l b^{\beta}\right)} \frac{\kappa^{4} \xi^{2} a^{\beta}}{432\left(-\kappa^{2} \xi\right)^{3 \beta}} \\
& \left(\frac{2-8 \kappa^{2} \xi \phi^{2}+12 \kappa^{4} \xi^{2} \phi^{4}+3 \beta-18 \beta^{2}-6 \beta \kappa^{2} \xi \phi^{2}-3 \kappa^{4} \xi^{2} \phi^{4} \beta-72 \beta^{2} \kappa^{4} \xi^{2} \phi^{4}+63 \beta^{2} \kappa^{2} \xi \phi^{2}}{\left(-1+\kappa^{2} \xi \phi^{2}\right)^{4}}+\right. \\
& \frac{27 \beta^{2} \kappa^{6} \xi^{3} \phi^{6}-27 \beta^{3}+27 \beta^{3} \kappa^{6} \xi^{3} \phi^{6}-81 \beta^{3} \kappa^{4} \xi^{2} \phi^{4}+81 \beta^{3} \kappa^{2} \xi \phi^{2}+6 \beta \kappa^{6} \xi^{3} \phi^{6}}{\left(-1+\kappa^{2} \xi \phi^{2}\right)^{4}}+3(1-3 \beta)\left(2+9 \beta^{2}\right. \\
& +9 \beta) \beta \grave{\mathcal{O}})-\sqrt{\frac{l-1}{\vartheta l}}\left\{\frac{\sqrt{6} \kappa^{2} b^{\beta} \beta^{-1}}{6 \vartheta l\left(-\kappa^{2} \xi\right)^{\frac{1}{2}+\beta}}\left(\frac{\grave{\mathcal{S}}\left(2 \kappa^{2} \xi \phi^{2}-3-2 \beta\right)}{\left(-1+\kappa^{2} \xi \phi^{2}\right)(2 \beta+1)}-\frac{1}{4} \grave{\mathcal{S}}(3+2 \beta) \grave{\mathcal{O}}\right)-[3(1+\beta)\right. \\
& \left(\frac{\grave{\mathcal{T}}\left(6 \kappa^{2} \xi \phi^{2} \beta+5 \kappa^{2} \xi \phi^{2}-6 \beta-3\right)}{\left(-1+\kappa^{2} \xi \phi^{2}\right)^{2}}+\frac{3}{2} \grave{\mathcal{T}}\left(8 \beta+1+12 \beta^{2}\right) \grave{\mathcal{O}}\right)+\left(\frac{3}{2} \grave{U}\left(5+46 \beta+108 \beta^{2}+72 \beta^{3}\right) \grave{\mathcal{O}}\right. \\
& +\frac{\grave{\mathcal{U}}\left(33 \kappa^{4} \xi^{2} \phi^{4}-40 \kappa^{2} \xi \phi^{2}+48 \beta+15-108 \kappa^{2} \xi \phi^{2} \beta-72 \beta^{2} \kappa^{2} \xi \phi^{2}+60 \beta \kappa^{4} \xi^{2} \phi^{4}+36 \beta^{2} \kappa^{4} \xi^{2} \phi^{4}\right)}{\left(-1+\kappa^{2} \xi \phi^{2}\right)^{3}} \\
& \left.\left.\left.+\frac{36 \beta^{2} \grave{\mathcal{U}}}{\left(-1+\kappa^{2} \xi \phi^{2}\right)^{3}}\right)\right] \frac{\sqrt{6} \kappa^{4} \xi}{108 \vartheta^{3} l^{3} \beta\left(-\kappa^{2} \xi\right)^{\frac{1}{2}+3 \beta}}\right\}+\frac{\kappa^{2}(l-1)}{6 \vartheta^{3} l^{3} \beta\left(-\kappa^{2} \xi\right)^{2 \beta}}\left[\left(\grave{\mathcal{V}}+\frac{\grave{\mathcal{W}} b^{\beta}(1+\beta)}{2\left(1+3 \vartheta l^{2} b^{\beta}-3 \vartheta l b^{\beta}\right)}\right.\right. \\
& +\vartheta l \beta \grave{\mathcal{Z}})\left(\frac{\kappa^{2} \xi \phi^{2}-1-2 \beta}{\beta\left(-1+\kappa^{2} \xi \phi^{2}\right)}-(2+4 \beta) \grave{\mathcal{O}}\right)+\frac{\xi b^{\beta} \vartheta^{2} l^{2} \grave{\mathcal{X}}}{6\left(\frac{b^{-\beta}}{3 \vartheta l(l-1)}+1\right)}\left(\frac{\left(2 \beta \kappa^{2} \xi \phi^{2}-2 \beta+2 \kappa^{2} \xi \phi^{2}-1\right)}{\left(-1+\kappa^{2} \xi \phi^{2}\right)^{2}}\right. \\
& +2 \grave{\mathcal{X}} \beta(1+2 \beta) \grave{\mathcal{O}})]+\frac{1}{4} \kappa^{2} \phi^{2}+\frac{1}{4} \frac{\kappa^{2} \phi^{2}}{\beta}+\left.\frac{1}{4} \frac{\ln \left(-1+\kappa^{2} \xi \phi^{2}\right)}{\xi \beta}\right|_{h c} ^{f},
\end{aligned}
$$

$$
\grave{\vartheta}=\frac{\phi^{1+4 \beta}\left(-\kappa^{2} \xi\right)^{5 / 2+2 \beta}(5+4 \beta)}{4(5 / 2+2 \beta) \kappa^{4} \xi^{2}}, \quad \grave{\mathcal{B}}=\frac{\phi^{1+2 \beta}\left(-\kappa^{2} \xi\right)^{\beta+3 / 2}(-2 \beta-3)}{(\beta+3 / 2) \kappa^{2} \xi}, \quad \grave{\mathcal{C}}=\frac{\phi^{4 \beta}\left(-\kappa^{2} \xi\right)^{2 \beta}(1+\beta)}{2+2 \beta}
$$

$$
\grave{\mathcal{D}}=\frac{\phi^{1+6 \beta}\left(-\kappa^{2} \xi\right)^{5 / 2+3 \beta}(5+6 \beta)}{4(5 / 2+3 \beta) \kappa^{4} \xi^{2}}, \quad \grave{\mathcal{E}}=\frac{\phi^{1+6 \beta}\left(-\kappa^{2} \xi\right)^{5 / 2+3 \beta}(5+6 \beta)}{8(5 / 2+3 \beta) \kappa^{4} \xi^{2}}, \quad \grave{\mathcal{F}}=\frac{\phi^{4 \beta+1}\left(-\kappa^{2} \xi\right)^{7 / 2+2 \beta}(7+4 \beta)}{8(7 / 2+2 \beta) \kappa^{6} \xi^{3}}
$$

$$
\grave{\mathcal{G}}=\frac{\phi^{8 \beta+1}\left(-\kappa^{2} \xi\right)^{4 \beta+7 / 2}(8 \beta+7)}{16(4 \beta+7 / 2) \kappa^{6} \xi^{3}}, \quad \grave{\mathcal{H}}=\frac{\phi^{8 \beta+1}\left(-\kappa^{2} \xi\right)^{4 \beta+7 / 2}(8 \beta+7)}{16(4 \beta+7 / 2) \kappa^{6} \xi^{3}}, \quad \grave{\mathcal{K}}=\frac{\phi^{8 \beta+1}\left(-\kappa^{2} \xi\right)^{9 / 2+4 \beta}(9+8 \beta)}{32(9 / 2+4 \beta) \kappa^{8} \xi^{4}}
$$

$$
\grave{\mathcal{O}}=\operatorname{LerchPhi}\left(\kappa^{2} \xi \phi^{2}, 1, \beta+\frac{1}{2}\right), \quad \grave{\mathcal{I}}=\frac{8 \phi^{8 \beta}\left(-\kappa^{2} \xi\right)^{4 \beta}(\beta+1)}{4 \beta+4}, \quad \grave{\mathcal{J}}=\frac{3 \phi^{6 \beta}\left(-\kappa^{2} \xi\right)^{3 \beta}(\beta+1)}{3 \beta+3}
$$




$$
\grave{\mathcal{Q}}=\frac{3 \phi^{6 \beta}\left(-\kappa^{2} \xi\right)^{3 \beta}(\beta+1)}{3 \beta+3}, \quad \grave{\mathcal{R}}=\frac{3 \phi^{6 \beta}\left(-\kappa^{2} \xi\right)^{3 \beta}(\beta+1)}{3 \beta+3}, \quad \grave{\mathcal{S}}=\frac{\phi^{2 \beta+1}\left(-\kappa^{2} \xi\right)^{\beta+5 / 2}(5+2 \beta)}{2(\beta+5 / 2) \kappa^{4} \xi^{2}}
$$

$$
\grave{\mathcal{T}}=\frac{\phi^{6 \beta+1}\left(-\kappa^{2} \xi\right)^{3 \beta+5 / 2}(6 \beta+5)}{4(3 \beta+5 / 2) \kappa^{4} \xi^{2}}, \quad \grave{\mathcal{U}}=\frac{\phi^{6 \beta+1}\left(-\kappa^{2} \xi\right)^{3 \beta+7 / 2}(6 \beta+7)}{8(3 \beta+7 / 2) \kappa^{6} \xi^{3}}, \quad \grave{\mathcal{V}}=\frac{\phi^{4 \beta}\left(-\kappa^{2} \xi\right)^{2 \beta}(\beta+1)}{2 \beta+2}
$$

$$
\grave{\mathcal{W}}=\frac{\phi^{4 \beta}\left(-\kappa^{2} \xi\right)^{2 \beta}(\beta+1)}{2 \beta+2}, \quad \grave{\mathcal{X}}=\frac{2 \phi^{4 \beta}\left(-\kappa^{2} \xi\right)^{2 \beta}(\beta+1)}{2 \beta+2}, \quad \grave{\mathcal{Z}}=\frac{\phi^{4 \beta}\left(-\kappa^{2} \xi\right)^{2 \beta}(\beta+1)}{2 \beta+2}
$$

\section{Appendix B: Eigenvalues corresponding to critical} line $\mathcal{L}_{1}$

$$
\begin{aligned}
& \lambda_{2 \mathcal{L}_{1}}=\frac{3}{2\left(1-x^{2}-z^{2}+z^{2} x^{2}\right)}\left(-y^{2} \sqrt{1-x^{2}}\right. \\
& +y^{2} x^{2} \sqrt{1-x^{2}}+1-x^{2}-2 z^{2}+2 z^{2} x^{2}+\omega-\omega x^{2} \\
& -2 \omega z^{2}+2 \omega z^{2} x^{2}+y^{2} z^{2} \sqrt{1-x^{2}}-y^{2} \omega \sqrt{1-x^{2}} \\
& \left.+y^{2} \omega z^{2} \sqrt{1-x^{2}}+z^{4}-z^{4} x^{2}+z^{4} \omega-z^{4} \omega x^{2}\right) \text {, } \\
& \lambda_{3 \mathcal{L}_{1}, 4 \mathcal{L}_{1}}=\frac{-1}{4\left(1-x^{2}-z^{2}+z^{2} x^{2}\right)}\left(-21 x^{2}-18 z^{2} x^{4}\right. \\
& -3 z^{4} \omega+18 x^{4}-3 \omega-3 z^{4}-6 \omega z^{2} x^{2}+3+12 \sqrt{3} y x^{3} z^{2} \\
& +6 \omega z^{2}-12 \sqrt{3} y x z^{2}-9 \sqrt{1-x^{2}} y^{2} \omega z^{2}+18 z^{2} x^{2}+3 \omega x^{2} \\
& +3 z^{4} x^{2}+9 \sqrt{1-x^{2}} y^{2}-9 \sqrt{1-x^{2}} y^{2} z^{2}-9 \sqrt{1-x^{2}} y^{2} x^{2} \\
& -12 \sqrt{3} y x^{3}+3 z^{4} \omega x^{2}+12 \sqrt{3} y x+9 \sqrt{1-x^{2}} y^{2} \omega \\
& \pm\left[81-81 y^{4} \omega^{2} z^{4} x^{2}+162 y^{4} \omega^{2} z^{2} x^{2}+81 y^{4}-162 y^{4} \omega z^{4} x^{2}\right. \\
& -162 y^{4} \omega z^{2} x^{4}-672 x^{2} y^{2} z^{2}+486 y^{4} \omega z^{2} x^{2}-486 x^{2}-216 z^{2} \\
& -96 y^{2}-972 x^{6}-72 z^{6}+336 x^{2} y^{2} z^{4}+144 y^{2} x^{6} z^{4}+9 \omega^{2} \\
& +324 x^{8}-756 z^{4} \omega x^{2}+864 z^{4} \omega x^{4}+684 \omega z^{2} x^{2}+768 y^{2} x^{4} z^{2} \\
& -828 \omega z^{2} x^{4}+324 \omega z^{2} x^{6}-324 z^{4} \omega x^{6}-288 y^{2} x^{6} z^{2} \\
& -324 y^{4} \omega z^{2}+162 y^{4} \omega z^{4}-162 y^{4} \omega^{2} z^{2}+81 y^{4} \omega^{2} z^{4} \\
& +324 y^{4} z^{2} x^{2}-324 y^{4} \omega x^{2}+324 z^{6} \omega x^{2}-324 z^{6} x^{4} \omega \\
& +108 z^{6} x^{6} \omega-162 y^{4} z^{2} x^{4}+162 y^{4} \omega x^{4}-81 y^{4} z^{4} x^{2} \\
& -36 \omega^{2} z^{2} x^{4}-81 y^{4} \omega^{2} x^{2}+54 \omega^{2} z^{4} x^{4}-36 \omega^{2} z^{6} x^{4}
\end{aligned}
$$

$$
\begin{aligned}
& +72 \omega^{2} z^{2} x^{2}-108 \omega^{2} z^{4} x^{2}+72 \omega^{2} z^{6} x^{2}+9 z^{8} \omega^{2} x^{4} \\
& -36 z^{8} \omega x^{2}+18 z^{8} \omega x^{4}-18 z^{8} \omega^{2} x^{2}-384 z^{4} x^{4} y^{2} \\
& +144 \sqrt{3} y x^{3} \omega z^{2}-144 \sqrt{3} y x^{3} z^{4} \omega+72 \sqrt{3} y x z^{4} \omega \\
& -72 \sqrt{3} y x \omega z^{2}-1296 \sqrt{1-x^{2}} y^{2} x^{2} \omega z^{2}+1053 x^{4} \\
& +120 \sqrt{3} y^{3} x^{3} z^{4} \sqrt{1-x^{2}}-120 \sqrt{3} \sqrt{1-x^{2}} y^{3} x \omega \\
& +144 \sqrt{3} \sqrt{1-x^{2}} y^{3} x z^{2}-120 \sqrt{3} y^{3} x z^{4} \sqrt{1-x^{2}} \\
& -168 \sqrt{3} y^{3} x^{3} z^{2} \sqrt{1-x^{2}}+24 \sqrt{3} x^{5} y^{3} z^{2} \sqrt{1-x^{2}} \\
& -144 z^{4} x^{7} \sqrt{3} y-72 \omega z^{2} x^{5} \sqrt{3} y-24 \sqrt{3} x^{5} y z^{6} \omega \\
& +48 z^{6} \sqrt{3} y x^{3} \omega-162 \omega^{2} z^{2} x^{2} \sqrt{1-x^{2}} y^{2} \\
& -378 z^{4} x^{4} y^{2} \sqrt{1-x^{2}} \omega+756 y^{2} x^{4} z^{2} \sqrt{1-x^{2}} \omega \\
& +162 \omega^{2} z^{4} x^{2} \sqrt{1-x^{2}} y^{2}+810 z^{4} \omega x^{2} y^{2} \sqrt{1-x^{2}} \\
& -108 z^{6} \sqrt{1-x^{2}} y^{2} \omega x^{2}-54 \sqrt{1-x^{2}} y^{2} \omega^{2} z^{6} x^{2} \\
& -24 z^{6} \sqrt{3} y x \omega+120 \sqrt{3} \sqrt{1-x^{2}} y^{3} x^{3} \omega+72 z^{4} \omega x^{5} \sqrt{3} y \\
& +624 \sqrt{3} y x^{3} z^{2}+240 \sqrt{3} \sqrt{1-x^{2}} y^{3} x \omega z^{2}-168 \sqrt{3} y x z^{2} \\
& +120 \sqrt{3} y^{3} x^{3} z^{4} \sqrt{1-x^{2}} \omega-240 \sqrt{3} y^{3} x^{3} z^{2} \sqrt{1-x^{2}} \omega \\
& +24 \sqrt{3} y x \omega-120 \sqrt{3} y^{3} x z^{4} \sqrt{1-x^{2}} \omega+120 \sqrt{3} y x z^{4} \\
& +540 \sqrt{1-x^{2}} y^{2} \omega z^{2}-744 \sqrt{3} x^{5} y z^{2}-384 \sqrt{3} y x^{3} z^{4} \\
& +594 \sqrt{1-x^{2}} y^{2} x^{2} \omega-1242 \sqrt{1-x^{2}} y^{2} x^{2} z^{2} \\
& -48 \sqrt{3} y x^{3} \omega-24 \sqrt{3} \sqrt{1-x^{2}} y^{3} x+48 \sqrt{3} \sqrt{1-x^{2}} y^{3} x^{3} \\
& +1188 y^{2} x^{4} z^{2} \sqrt{1-x^{2}}-378 z^{4} x^{4} y^{2} \sqrt{1-x^{2}}+54 \omega \\
& +408 z^{4} x^{5} \sqrt{3} y+288 z^{2} x^{7} \sqrt{3} y-324 z^{2} x^{6} \sqrt{1-x^{2}} y^{2} \\
& -378 \sqrt{1-x^{2}} y^{2} x^{4} \omega+162 \sqrt{1-x^{2}} y^{2} \omega^{2} z^{2}+9 z^{8} \\
& +48 z^{6} \sqrt{3} y x^{3}-24 z^{6} \sqrt{3} y x+108 z^{6} \sqrt{1-x^{2}} y^{2} \omega \\
& -432 z^{4} \sqrt{1-x^{2}} y^{2} \omega+648 z^{4} \sqrt{1-x^{2}} y^{2} x^{2} \\
& -24 \sqrt{3} x^{5} y z^{6}-162 \sqrt{1-x^{2}} y^{2} \omega^{2} z^{4}+24 \omega x^{5} \sqrt{3} y
\end{aligned}
$$




$$
\begin{gathered}
+54 \sqrt{1-x^{2}} y^{2} \omega^{2} z^{6}+54 \omega^{2} x^{2} \sqrt{1-x^{2}} y^{2} \\
-54 z^{6} x^{2} y^{2} \sqrt{1-x^{2}}-24 \sqrt{3} x^{5} y^{3} \sqrt{1-x^{2}}-36 \omega^{2} z^{6} \\
-144 x^{7} \sqrt{3} y+324 x^{6} \sqrt{1-x^{2}} y^{2}+378 \sqrt{1-x^{2}} y^{2} z^{2} \\
+648 \sqrt{1-x^{2}} y^{2} x^{2}-216 \sqrt{1-x^{2}} y^{2} \omega+9 z^{8} \omega^{2} \\
+72 \sqrt{3} y x-288 \sqrt{3} y x^{3}-54 \sqrt{1-x^{2}} y^{2} \omega^{2} \\
+54 z^{6} \sqrt{1-x^{2}} y^{2}-270 z^{4} \sqrt{1-x^{2}} y^{2}+360 \sqrt{3} x^{5} y \\
-810 \sqrt{1-x^{2}} y^{2} x^{4}-162 \sqrt{1-x^{2}} y^{2}-2376 z^{2} x^{4} \\
-180 \omega z^{2}+1188 z^{2} x^{2}-936 z^{4} x^{2}+198 z^{4}+216 z^{4} \omega \\
+192 y^{2} z^{2}+336 x^{2} y^{2}+144 y^{2} x^{6}+2052 z^{2} x^{6}+18 z^{8} \omega \\
+270 \omega x^{4}-108 \omega x^{6}+1602 z^{4} x^{4}-1188 z^{4} x^{6}+81 y^{4} \omega^{2} \\
+162 y^{4} \omega-162 y^{4} z^{2}-108 z^{6} \omega-288 z^{6} x^{4}+108 z^{6} x^{6} \\
-243 y^{4} x^{2}+81 y^{4} z^{4}+252 z^{6} x^{2}+243 y^{4} x^{4}-81 y^{4} x^{6} \\
-96 y^{2} z^{4}-36 \omega^{2} z^{2}+54 \omega^{2} z^{4}-648 x^{8} z^{2}-18 \omega^{2} x^{2} \\
+9 \omega^{2} x^{4}-18 z^{8} x^{2} \\
+9 z^{8} x^{4}+324 z^{4} x^{8}-216 \omega x^{2} \\
\left.-384 y^{2} x^{4}\right]^{\frac{1}{2}}
\end{gathered}
$$

[1] A. Guth, Phys. Rev. D, 62, 105030, (1981).

[2] A. D. Linde, Phys. Lett. B, 108, 389 (1982)

[3] A. Albrecht and P. Steinhard, Phys. Rev. D, 48, 1220, (1982)

[4] A. D. Linde, Particle Physics and Inflationary Cosmology (Harwood Academic Publishers, Chur, Switzerland, 1990).

[5] A. Liddle and D. Lyth, Cosmological Inflation and LargeScale Structure, (Cambridge University Press, 2000).

[6] J. E. Lidsey et al, Phys. Rev. D, 69, 373, (1997).

[7] A. Riotto, arXiv:hep-ph/0210162

[8] D. H. Lyth and A. R. Liddle, The Primordial Density Perturbation (Cambridge University Press, 2009).

[9] R. H. Brandenberger, arXiv:hep-th/0509099.

[10] A. G. Riess, et al., Astrophys. J., 116, 1009, (1998).

[11] A. G. Riess et al., Astrophys. J., 607, 665, (2004).

[12] V. Springel, C. S. Frank and S. M. D. White, Nature (London), 440, 1137, (2006).

[13] T. Padmanabhan, Phys. Rep, 380, 235, (2003).

[14] S. M. Carroll, Living Rev. Relativity, 4, 1, (2001).

[15] E. J. Copeland, M. Sami and S. Tsujikawa, Int. J. Mod. Phys. D, 15, 1753, (2006).

[16] F. Piazza, \& S. Tsujikawa, JCAP, 07, 004, (2004).

[17] H. Wei, \& R. G. Cai, Phys. Rev. D, 73, 083002, (2006).

[18] A. Anisimov, E. Babichev and A. Vikman, JCAP, 06, 006, (2005).

[19] B. Wang et al., Phys. Lett. B, 624, 141, (2005).

[20] S. Nojiri and S. D. Odintsov, Gen. Rel. Grav, 38, 1285, (2006).

[21] W. Zhao, Phys. Rev. D, 73, 123509, (2006).

[22] U. Alam, V. Sahni and A. A. Starobinsky, JCAP, 06,
008, (2004).

[23] M. Sami, arXiv:0901.0756, (2009).

[24] G. Caldera-Cabral, R. Maartens and L. A. Urena-Lopez, Phys. Rev. D, 79, 063518, (2009).

[25] V. Sahni, Lect. Notes Phys., 653, 141, (2004).

[26] H. Zhang, arXiv:0909.3013., (2009).

[27] S. Capozziello, V. F. Cardone, S. Carloni and A. Troisi, Int. J. Mod. Phys. D, 12, 1969, (2003).

[28] S. Nojiri and S. D. Odintsov, AIP Conf. Proc., 1115, 212, (2009).

[29] T. P. Sotiriou and V. Faraoni, Rev. Mod. Phys, 82, 451, (2010).

[30] L. Amendola, D. Polarski and S. Tsujikawa, Phys. Rev. Lett., 98, 131302, (2007).

[31] S. D. Tsujikawa, Lect. Notes Phys., 800, 99, (2010).

[32] R. R. Caldwell, R.Dave and P. J. Steinhardt, Phys. Rev. Lett., 80, 1582, (1998).

[33] A. R. Liddle and R. J. Scherrer, Phys. Rev. D, 59, 023509, (1999).

[34] B. Ratra and P. J. E. Peebles, Phys. Rev. D, 37, 3406, (1988).

[35] A. Sen, JHEP, 9910, 008, (1999).

[36] A. Sen, JHEP, 0207, 065, (2002).

[37] A. Sen, Mod. Phys. Lett. A, 17, 1797, (2002).

[38] M. Sami, P. Chingangbam and T. Qureshi, Phys. Rev. D, 66, 043530, (2002).

[39] A. Feinstein, Phys. Rev. D, 66, 063511 (2002).

[40] T. Padmanabhan, Phys. Rev. D, 66, 021301 (2002).

[41] V. Gorini, A.Y. Kamenshchik, U. Moschella and V. Pasquier, Phys. Rev. D, 69, 123512 (2004).

[42] E. J. Copeland, M. R. Garousi, M. Sami and S.Tsujikawa, 
Phys. Rev. D, 71, 043003, (2005).

[Gibbons] G. W. Gibbons, Phys. Lett. B, 537, 1, (2002).

[43] V. Faraoni, Phys. Rev. D, 53, 6813, (1996).

[44] V. Faraoni, Phys. Rev. D, 62, 023504, (2000).

[45] B. L. Spokoiny, Phys. Lett. B, 147, 39 (1984).

[46] T. Futamase and K. I. Maeda, Phys. Rev. D, 39, 399, (1989).

[47] D. S. Salopek, J. R. Bond and J. M. Bardeen, Phys. Rev. D, 40, 1753, (1989).

[48] R. Fakir and W. G. Unruh, Phys. Rev. D, 41, 1783, (1990).

[49] N. Makino and M. Sasaki, Prog. Theor. Phys., 86, 103, (1991)

[50] J. Hwang and H. Noh, Phys. Rev. D, 60, 123001, (1999).

[51] S. Tsujikawa and H. Yajima, Phys. Rev. D, 62, 123512, (2000).

[52] C. Pallis and N. Toumbas, JCAP, 1102, 019, (2011).

[53] K. Nozari, JCAP, 0709, 003, (2007).

[54] K. Nozari and S. Shafizadeh, Phys. Scr. 82, 015901, (2010).

[55] K. Nozari and N. Rashidi, Phys. Rev. D, 86, 043505, (2012).

[56] Y-S. Piao, Q-G. Huang, X. Zhang and Y-Z. Zhang, Phys. Lett. B, 570, 1, (2003).

[57] P. Chingangbam, S. Panda and A. Deshamukhya, JHEP, 0502, 052, (2005).

[58] G. L. Alberghi and A. Tronconi, arXiv:hep-ph/0509044], (2005).

[59] J. Bardeen, Phys. Rev. D, 22, 1882, (1980).

[60] V. F. Mukhanov, H. A. Feldman and R. H. Brandenberger, Phys. Rept., 215, 203, (1992).

[61] E. Bertschinger, arXiv:astro-ph/9503125, (1995).
[62] D. Langlois, R. Maartens and D. Wands, Phys. Lett. B, 489, 259, (2000).

[63] D. Langlois and F. Vernizzi, JCAP, 02, 017, (2007).

[64] B. A. Bassett, F. Tamburini, D. I. Kaiser and R. Maartens, Nucl. Phys. B, 561, 188, (1999).

[65] C. Gordon, D. Wands, B. A. Basset and R. Maartens, Phys. Rev. D, 63, 023506, (2001).

[66] M. Bouhamdi-Lopez, R. Maartens and D. Wands, Phys. Rev. D, 70, 123519, (2004).

[67] N. Kaloper, Phys. Rev. D, 71, 086003, (2005).

[68] R. Maartens, D. Wands, B. A. Bassett and I. Heard, Phys. Rev. D, 62, 041301, (2000).

[69] J. M. Bardeen, P. J. Steinhardt and M. S. Turner, Phys. Rev. D, 28, 679, (1983).

[70] D. Wands, K. A. Malik, D. H. Lyth and A. R. Liddle, Phys. Rev. D, 62, 043527, (2000).

[71] A. R. Liddle and D. H. Lyth, Phys. Rep. 231, 1, (1993).

[72] G. Hinshaw et al., arXiv:1212.5226, (2013).

[73] S. del Campo, R. Herrera nad A. Toloza, Phys. Rev. D, 79, 083507, (2009).

[74] S. Gupta, A. Berera, A. F. Heavens and S. Matarrese, Phys. Rev. D, 66, 043510, (2002).

[75] T. Falk, R. Rangarajan and M. Srednicki, ApJ Lett., 403, 1, (1993).

[76] V. Sahni, T. D. Saini, A. A. Starobinsky and U. Alam, JETP Lett. 77, 201, (2003).

[77] U. Alam, V. Sahni, T. D. Saini and A. A. Starobinsky, Mon. Not. Roy. ast. Soc. 344, 1057, (2003).

[78] L. Amendola, C. Charmousis and S. C. Davis, JCAP 0612, 020, (2006).

[79] L. Amendola, C. Charmousis and S. C. Davis, JCAP 0710, 004, (2007). 